\title{
A New Analysis Technique to Improve Spatial Resolution for a Resistive Anode Time Projection Chamber
}

\author{
by \\ Nicholi M. Shiell

\begin{abstract}
A Thesis submitted to
the Faculty of Graduate and Postdoctoral Affairs

in partial fulfilment of

the requircments for the degree of

Master of Science
\end{abstract} \\ Ottawa-Carleton Institute for \\ Physics \\ Department of Physics \\ Carleton University \\ Ottawa, Ontario, Canada \\ July 23,2012
}

Copyright (C)

2012 - Nicholi M. Shiell 
Library and Archives

Canada

Published Heritage

Branch

395 Wellington Street

Ottawa ON K1A ON4

Canada
Bibliothèque et

Archives Canada

Direction du

Patrimoine de l'édition

395 , rue Wellington

Ottawa ON K1A ON4

Canada
Your file Votre référence

ISBN: 978-0-494-93544-6

Our file Notre référence

ISBN: $978-0-494-93544-6$
NOTICE:

The author has granted a nonexclusive license allowing Library and Archives Canada to reproduce, publish, archive, preserve, conserve, communicate to the public by telecommunication or on the Internet, loan, distrbute and sell theses worldwide, for commercial or noncommercial purposes, in microform, paper, electronic and/or any other formats.

The author retains copyright ownership and moral rights in this thesis. Neither the thesis nor substantial extracts from it may be printed or otherwise reproduced without the author's permission.
AVIS:

L'auteur a accordé une licence non exclusive permettant à la Bibliothèque et Archives Canada de reproduire, publier, archiver, sauvegarder, conserver, transmettre au public par télécommunication ou par l'Internet, prêter, distribuer et vendre des thèses partout dans le monde, à des fins commerciales ou autres, sur support microforme, papier, électronique et/ou autres formats.

L'auteur conserve la propriété du droit d'auteur et des droits moraux qui protege cette thèse. $\mathrm{Ni}$ la thèse ni des extraits substantiels de celle-ci ne doivent être imprimés ou autrement reproduits sans son autorisation.
In compliance with the Canadian Privacy Act some supporting forms may have been removed from this thesis.

While these forms may be included in the document page count, their removal does not represent any loss of content from the thesis.
Conformément à la loi canadienne sur la protection de la vie privée, quelques formulaires secondaires ont été enlevés de cette thèse.

Bien que ces formulaires aient inclus dans la pagination, il n'y aura aucun contenu manquant. 


\section{Abstract}

A $2 \mathrm{~m}$ long Time Projection Chamber (TPC) is being developed as the central tracker for the proposed International Large Detector (ILD) at the Intenational Linear Collider (ILC). In order to achieve the momentum resolution required by the ILD, the single hit transverse resolution must not exceed $100 \mu \mathrm{m}$ at the full $2 \mathrm{~m}$ drift. These resolution requirements exceed what is possible with a traditional multi-wire TPC.

A TPC readout using Micro-Pattern Gas Detectors (MPGDs), with $\sim 1 \mathrm{~mm}$ wide readout pads is capable of achieving the resolution requirements. However, $\sim 1 \mathrm{~mm}$ pads increases the number of readout channels and detector complexity. The use of wider pads is possible using the concept of charge dispersion first developed by our group at Carleton.

Beam tests of a Large Prototype TPC (LP-TPC) with charge dispersion have shown the ILC requirements can be achieved using $\sim 3 \mathrm{~mm}$ wide pads. However, these measurements required relatively long (500 ns) shaping times for the digitized main amplifier pulse, and this will degrade the TPC longitudinal resolution and two hit resolving power.

For my thesis research, I have developed a new pulse analysis technique which improves transverse resolution while employing short (100 ns) shaping time amplifier pulses. Using this new technique the transverse resolution requirements, at ILC conditions, can be achieved without compromising the TPC longitudinal resolution or two hit resolving power. 


\section{Acknowledgments}

I would like to thank the following people without whose help, support, and encouragement this work could not have been completed. Firstly, I would like to thank my supervisor Dr. Madhu Dixit for teaching me almost everything I know about TPCs and for having the patience to work with me especially throughout the writing of this document. I would also like to thank our collaborators from Saclay France, Paul Colas, David Attie, and Wenxin Wang for their help at the beam test and throughout the course of this work. I also owe thanks to the ILC group members at Carleton, Alain Bellerive, Peter Hayman, and especially Yun-Ha Shin for teaching how to use the analysis code and answering my many questions about it. Lastly I would like to thank my friends and family for their support and encouragement throughout my

Masters. I would especially like to thank my sister Sofika for helping draw many of the diagrams in this document.

I also owe thanks to the following collaborations: The Saclay group for the fabrication of the MicroMeGas modules and the AFTER readout electronics used during the test, the KEK group for providing us with the superconducting magnet, DESY laboratory for providing the electron test beam, and finally EUDET for providing infrastructure support for the beam test. 


\section{Table of Contents}

Abstract

Acknowledgments $\quad$ iv

Table of Contents $\quad$ i

List of Tables $\quad$ iv

List of Figures $\quad$ v

1 Introduction 2

1.1 The International Linear Collider (ILC) . . . . . . . . . . . . . 2

1.2 The International Large Detector for the ILC . . . . . . . . . 4

1.3 The International Large Detector Time Projection Chamber (ILD-TPC) 6

1.4 Motivation ....................... 8

1.5 Thesis Layout . . . . . . . . . . . . . . . . . . . . 9 9

1.6 Authors Contribution . . . . . . . . . . . . . . . . 10

2 Physics of a TPC $\quad 12$

2.1 Fundamentals of a TPC . . . . . . . . . . . . . . 12

2.2 Ionization and Drift . . . . . . . . . . . 15

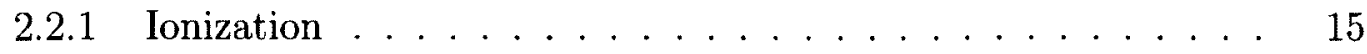

2.2 .2 Drift . . . . . . . . . . . . . . 17 
2.3 Traditional Wire TPC $\ldots \ldots \ldots \ldots \ldots$

2.3 .1 Wire Amplification . . . . . . . . . . . . . . . . 19

2.3 .2 Traditional Wire TPC Readout $\ldots \ldots \ldots \ldots . \ldots . \ldots 22$

2.4 The Resistive Anode MPGD TPC . . . . . . . . . . . . . . . . 23

2.4.1 Amplification by MircoMeGaS . . . . . . . . . . . . 23

2.4.2 Charge Dispersion Readout . . . . . . . . . . . 25

3 Experimental Setup and Data Taking 29

3.1 Experimental Setup . . . . . . . . . . . . . . . . . . . . . . 29

3.2 Readout Electronics . . . . . . . . . . . . . . . . . . 35

3.3 Data Taking . . . . . . . . . . . . . . . . . . 37

4 Analysis $\quad 39$

4.1 Overview of Analysis . . . . . . . . . . . . . . . . . . . 39

4.2 Track Fitting $\ldots \ldots \ldots \ldots \ldots \ldots \ldots \ldots \ldots \ldots$

4.3 Analysis Pipeline $\ldots \ldots \ldots \ldots$. . . . . . . . . . . . 44

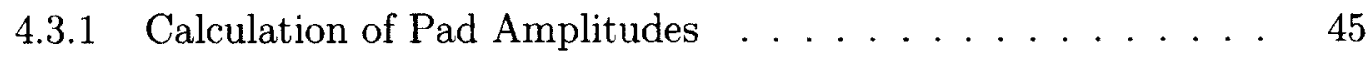

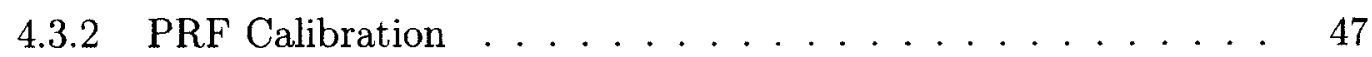

4.3 .3 Bias Corrections . . . . . . . . . . . . . . 52

4.4 Resolution Measurement . . . . . . . . . . . . . . . 57

4.5 Dependence of Resolution on Drift Distance . . . . . . . . . 58

5 Results $\quad 62$

5.1 Optimization of Integration Width $\ldots \ldots \ldots \ldots \ldots$

5.2 PRF Calibration $\ldots \ldots \ldots \ldots \ldots \ldots \ldots \ldots \ldots \ldots$

5.3 Relative Tracking Efficiency . . . . . . . . . . . . . . . 67

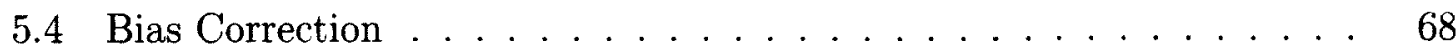

5.5 Resolution Measurements . . . . . . . . . . . . . . . 69 
5.6 Extrapolation of Resolution Results to ILC Conditions . . . . . . . .

$\begin{array}{llr}6 & \text { Conclusion } & 77\end{array}$

$\begin{array}{lr}\text { References } & 80\end{array}$

$\begin{array}{lll}\text { Appendix A Event Selection } & 83\end{array}$

A.1 Central Columns Check . . . . . . . . . . . . 83

A.2 Single Track Check . . . . . . . . . . . . . . . . . . 84

A.3 Event Veto . . . . . . . . . . . . . . . . 84

A.4 Amplitude Overflow . . . . . . . . . . . . . . . 84

A.5 Track Fit Failure . . . . . . . . . . . . . . . 85

A.6 Track Angle Cut . . . . . . . . . . . . . . . . . 85

$\begin{array}{ll}\text { Appendix B Scan and Run Parameters } & 86\end{array}$

$\begin{array}{lll}\text { Appendix C Resolution Definitions } & 88\end{array}$ 


\section{List of Tables}

1.1 ILD-TPC resolution requirements $\ldots \ldots \ldots \ldots \ldots$

3.1 Experimental setup parameters . . . . . . . . . . . 34

3.2 Amplifier shaper response function parameters . . . . . . . 36

3.3 Sample detector settings $\ldots \ldots \ldots \ldots$

5.1 Relative tracking efficiency $(\mathrm{QFM}) \ldots \ldots \ldots \ldots \ldots$

5.2 Relative tracking efficiency (Reintegration Method) . . . . . . . 71

5.3 Single hit $\mathrm{r} \phi$ resolution measurements . . . . . . . . . . . 74 


\section{List of Figures}

1.1 Layout of ILC facility . . . . . . . . . . . . . . 3

1.23 -D model of ILD detector system $\ldots \ldots \ldots \ldots \ldots$

1.3 Comparison of pad pulses for various shaping times $\ldots \ldots \ldots$

2.1 Basic TPC layout . . . . . . . . . . . . . . . 13

2.2 Identification of particles via $\mathrm{dE} / \mathrm{dx} \ldots \ldots \ldots \ldots \ldots$

2.3 Traditional TPC readout $\ldots \ldots \ldots \ldots \ldots \ldots$

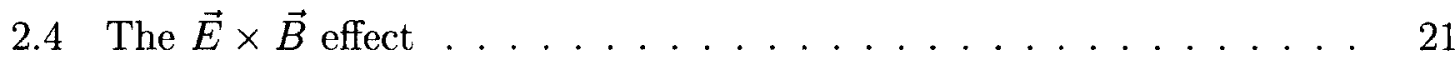

2.5 MicroMegas schematic . . . . . . . . . . . . . . . 24

2.6 Cross section view of resistive anode and MicroMegas $\ldots \ldots \ldots 27$

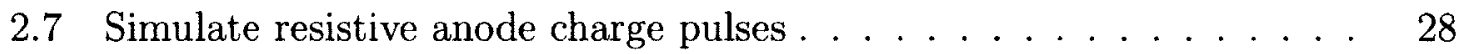

3.1 Diagram of the experimental setup $\ldots \ldots \ldots \ldots \ldots$

3.2 Photograph of the LP-TPC $\ldots \ldots \ldots \ldots \ldots \ldots$

3.3 Photograph of the LP-TPC in use. . . . . . . . . . . . . 32

3.4 Front view of the readout module $\ldots \ldots \ldots \ldots \ldots$

3.5 Shaper response functions for various shaping times . . . . . . . 36

3.6 Signal readout pipeline . . . . . . . . . . . . . . . . . 37

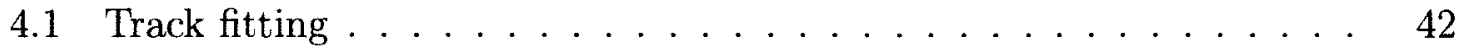

4.2 Analysis pipeline $\ldots \ldots \ldots \ldots \ldots \ldots \ldots \ldots$

4.3 Examples of QFM and SPM amplitude determination . . . . . 46

4.4 Example pedestal calculation used in Reintegration Method . . . . 48 
4.5 Example of RM amplitude determination . . . . . . . . . . . 48

4.6 PRF calibration flow chart . . . . . . . . . . . . . 49

4.7 Scatter plot used in PRF calibration . . . . . . . . . . . 51

4.8 Binned scatter plot used in PRF calibration . . . . . . . . . . 52

4.9 Example row residual scatter plot (before correction) . . . . . . . 56

4.10 Example averaged row residual plot (before correction) . . . . . . . 57

4.11 Example row residual scatter plot (after correction) . . . . . . . 58

4.12 Example averaged row residual plot (after correction) . . . . . . . . 59

4.13 Example global residual distributions . . . . . . . . . . . . . . . 60

5.1 Determination of optimal integration width . . . . . . . . . 63

5.2 Calibration results of PRF mixing parameter $\mathrm{r}(\mathrm{QFM})$. . . . . . . 64

5.3 Calibration results of PRF width parameter w (QFM) . . . . . . 65

5.4 Calibration results of PRF mixing parameter $\mathrm{r}$ (Reintegration) . . . . 66

5.5 Calibration results of PRF width parameter $w$ (Reintegration) . . . 67

5.6 PRF Comparison (drift distance $10 \mathrm{~cm}$ ) . . . . . . . . . . 68

5.7 PRF Comparison (drift distance $55 \mathrm{~cm}$ ) . . . . . . . . . . . 69

5.8 Average and STD row residual comparison (before and after correction) 73

5.9 Single hit $\mathrm{r} \phi$ resolution vs. drift distance . . . . . . . . . . 75

5.10 Extrapolation of Reintegration Method resolution to ILC conditions . 76 


\section{List of Acronyms}

AFTER ASIC For TPC Electronic Read out

ASIC Application Specific Integrated Circuit

CEA Atomic and Alternative Energy Commission of France

DESY Deutsches Elektronen-Synchrotron

FADC Flash Analog to Digital Converter

GEM Gas Electron Multiplier

ILC International Linear Collider

ILD International Large Detector

LHC Large Hadron Collider

LINAC Linear Accelerator

LP-TPC Large Prototype Time Projection Chamber

MicroMeGas Micro-Mesh Gaseous Structure

MPGD Micro Pattern Gas Detector

PRF Pad Response Function

QFM Quadratic Fit Method

RM Reintegration Method

RTE Relative Tracking Efficiency

RMS Root Mean Square

SiD Silicon Detector

SPM Single Point Maximum

T2K Tokai to Kamioka neutrino experiment, Japan

TPC Time Projection Chamber 


\section{Chapter 1}

\section{Introduction}

\subsection{The International Linear Collider (ILC)}

The International Linear Collider (ILC) is a future electron/positron collider intended to complement the Large Hadron Collider (LHC). The ILC will be able to perform precision measurements of particles and physical phenomena discovered at the LHC. The increase in precision of measurements made at the ILC is a result of the nature of the particles being collided. The LHC, as the name implies, collides hadrons, which are not fundamental particles. Instead they are a mixture of quarks and gluons making any interaction between them a complicated multi-body interaction. The ILC, however, will collide fundamental particles, namely the lightest charged lepton, the electron, and its antiparticle, the positron. Because these are fundamental particles they have no internal structure and so any interaction between them is a much simpler two body collision.

Figure 1.1 shows a diagram of the basic layout of the ILC facility. The initial phase of the accelerator calls for a total footprint of $\sim 31 \mathrm{~km}$. This initial stage will have a total $e^{+} / e^{-}$center of mass collision energy $\left(\mathrm{E}_{c m}\right)$ of $500 \mathrm{GeV}(250 \mathrm{GeV}$ from each beam), and a peak luminosity of $2 \times 10^{34} \mathrm{~cm}^{-2} \mathrm{~s}^{-1}$, which is a measure of the number of collisions occurring at the interaction point. A second phase is planned to 
increase the $\mathrm{E}_{c m}$ to $1 \mathrm{TeV}$ with an additional $11 \mathrm{~km}$ of LINACs (linear accelerators) added to each beam.

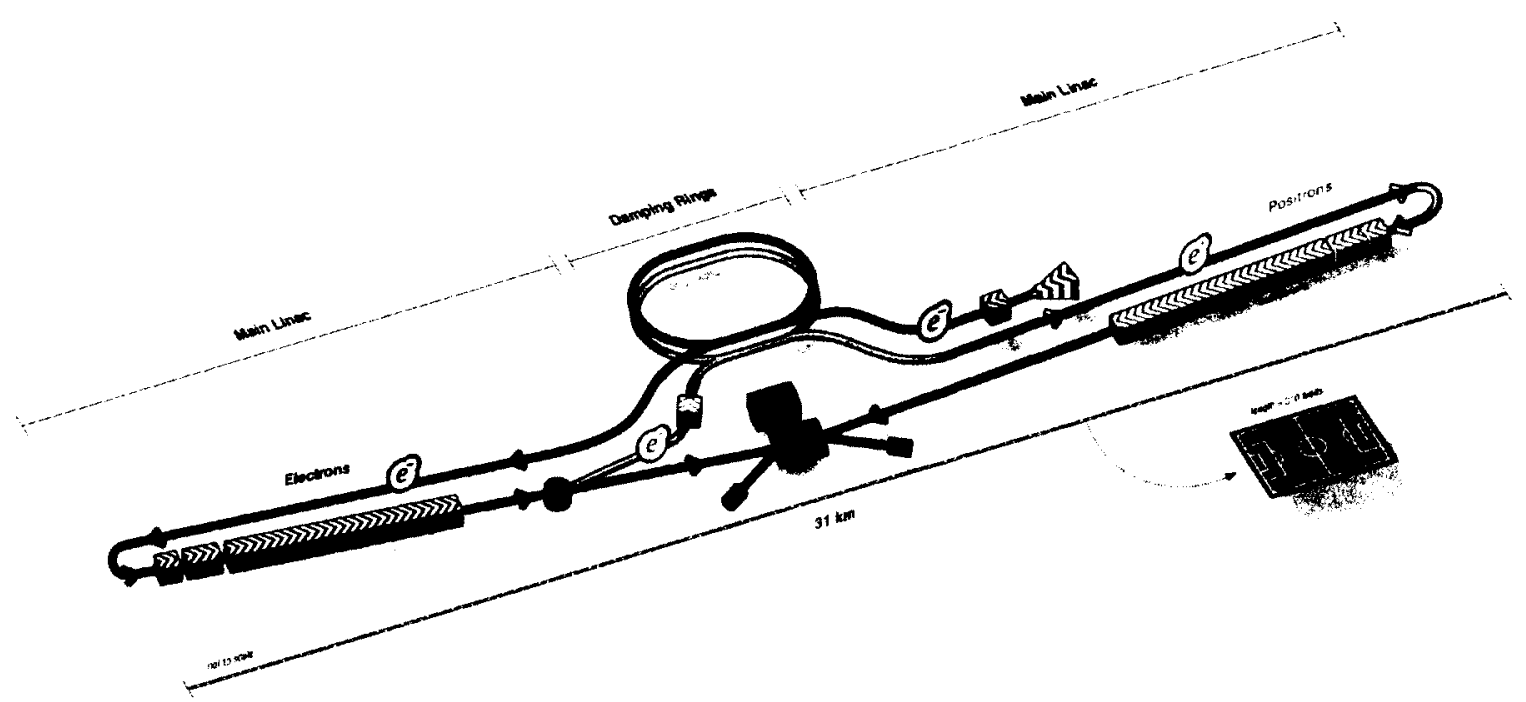

Figure 1.1: Diagram of the International Linear Collider layout. In the figure can be seen the electron and positron LINACs, the damping rings (used to compact the particle bunches), the positron/electron sources, and the beam crossing point with the detector system in place. Figure used with permission from the ILD collaboration.

Although the $\mathrm{E}_{c m}$ of the ILC is less than that of the LHC, the energy per particle is comparable. This is again a result of the nature of the particles being accelerated. The LHC energy is divided amongst the constituent quarks and gluons of each proton. The ILC's energy is entirely contained by a single electron or positron.

The ILC is intended to be a facility capable of probing some of the most important questions still open in particle physics. Central to a broad range of unanswered questions is the Higgs boson. The Standard Model of Particle Physics requires the existence of the Higgs boson to give mass to the other elementary particles. Recently a new boson has been discovered [1] at the LHC which is appears to be consistent with the Standard Model Higgs. Confirmation that this new boson is indeed the Higgs boson will require further studies of its physical properties and interactions. These studies will require the precision available at the ILC. 
Model independent studies of the Higgs boson can be conducted at the ILC using the so-called Golden Channel (see equation 1.1).

$$
e^{+} e^{-} \rightarrow Z^{0} H \rightarrow l^{+} l^{-} X
$$

where $Z^{0}$ is a $\mathrm{Z}$ boson, $l^{+} / l^{-}$are a lepton/anti-lepton pair and $\mathrm{X}$ are the decay products of the Higgs boson. The precision of Higgs studies conducted using the Golden Channel are limited by the knowledge of the beam energy and measurements of the final state lepton four momenta. The precision does not depend on the Higgs decay products, this is what is meant when the Golden Channel is said to be model independent. Current design requirements for ILC detector system concepts call for the precision of such a measurement to be ultimately limited by the beam energy. This means new detector technologies will be required to precisely measure the resulting leptons.

Currently there are two approved detector system concepts being developed to meet the requirements for use with the ILC. The first of these is SiD which is a silicon based detector. The other, the focus of the ILC group at Carleton, is the International Large Detector (ILD), which will be discussed further in the next section.

\subsection{The International Large Detector for the ILC}

The International Large Detector (ILD) is a detector system intended for use at the ILC (see figure 1.2). The design philosophy of the ILD, outlined in the 2010 Letter of Intent [2], is to build a detector which takes full advantage of the well defined initial state and relative simplicity of interactions provided by a lepton collider. To this end the ILD intends to couple high granularity calorimeters and a high precision charged particle tracking system. This will produce a final detector system with unprecedented spatial and energy resolution of the particle interactions limited 


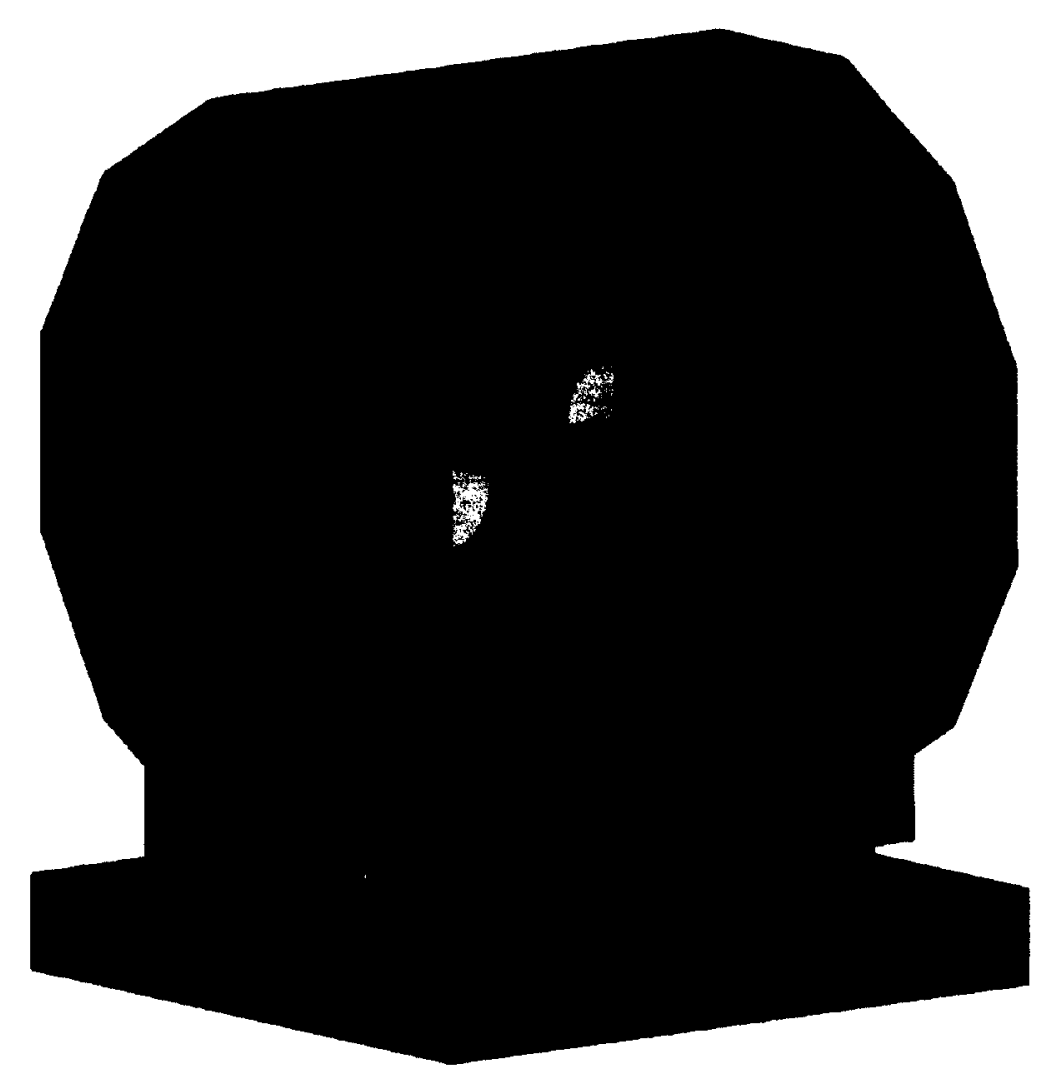

Figure 1.2: 3D model of the International Large Detector (ILD) system. The solenoidal magnet is shown in light brown enclosing the entire detector system. The beam pipe can be seen passing through the axis of the magnet. The Time Projection Chamber (TPC) is shown in yellow close to the beam pipe. Figure used with permission from the ILD collaboration [2].

only by the knowledge of the beam energy.

The ILD detector subsystems can be divided into three groups: the beam monitors, the detectors dedicated to measuring charged particle momenta and identifying particle type, and finally detectors intended to identify muons and measure the energy of electromagnetic and neutral particles resulting from interactions. All of these detectors can be seen in figure 1.2. Upstream of the interaction region in both beam directions, are the beam monitors. These are responsible for measuring the beam energy and polarization. Next come the detectors of the charged particle tracking 
system. These include a Pixel Vertex Detector, then a Silicon Strip Tracker. Surrounding these is the main component of the tracker, a Time Projection Chamber (TPC). Following the TPC are the detectors of the calorimetry group: first the electromagnetic calorimeter, then the hadronic calorimeter, and finally the muon chambers. There is also a forward calorimeter which give the system $4 \pi$ coverage.

The focus of the work conducted by the ILC group at Carleton, and the focus of this thesis, is the TPC. This detector is called the ILD-TPC and will be discussed in more detail in the next section.

\subsection{The International Large Detector Time Pro- jection Chamber (ILD-TPC)}

The International Large Detector Time Projection Chamber (ILD-TPC) is intended to be the central tracker for the ILD. The ILD design calls for a $4.3 \mathrm{~m}$ long cylindrical TPC, divided into two equal sized drift regions by a central cathode, with a diameter of $3.6 \mathrm{~m}$. This size of TPC will have a readout end plate surface area of $10 \mathrm{~m}^{2}$, which will need to be instrumented. These detector dimensions are taken from the latest TPC design report [3]. This large surface area is a driving force for the development of new readout technologies. The other driving forces are the unprecedented momentum resolution requirements.

To take full advantage of the possible precision at the ILC, the ILD places strict design requirements on the momentum resolution for the TPC. The ILD-TPC will have to significantly improve the single hit transverse $\left(\sigma_{r \phi}\right)$ and longitudinal $\left(\sigma_{r z}\right)$ spatial resolution over the traditional TPC design. The spatial resolution requirements are needed to achieve an order of magnitude improvement in momentum resolution. 
Apart from the momentum requirements, two track resolutions must also be improved. All the resolution requirements for the ILD-TPC are summarized in table 1.1. An explanation of the different spatial resolutions (single hit $\mathrm{r} \phi$ and $\mathrm{r} z$, as well as two track $\mathrm{r} \phi$ and $\mathrm{rz}$ ) and their effect on momentum resolution can be found in Appendix C.

\begin{tabular}{|c|c|c|}
\hline Resolution & Value & Unit \\
\hline$\delta\left(1 / p_{T}\right)$ & $2 \times 10^{-5}$ & $(\mathrm{GeV} / \mathrm{c})^{-1}$ \\
\hline$\sigma_{r \phi}$ & $<100$ & $\mu \mathrm{m}$ \\
\hline$\sigma_{r z}$ & $0.4-1.4$ & $\mathrm{~mm}$ \\
\hline 2 hit $\mathrm{r} \phi$ & $\sim 2$ & $\mathrm{~mm}$ \\
\hline $2 \mathrm{hit} \mathrm{rz}$ & $\sim 6$ & $\mathrm{~mm}$ \\
\hline $\mathrm{dE} / \mathrm{dx}$ & $\sim 5 \%$ & - \\
\hline
\end{tabular}

Table 1.1: Summary of ILD-TPC resolution requirements.

In order to achieve the design requirements of the ILD-TPC novel TPC readout technologies are being developed. The ILC group at Carleton has focused on the development of such a readout system. This novel readout utilizes a new class of amplification structure called Micro Pattern Gas Detectors (MPGDs). MPGDs have been shown to achieve resolutions on the order of $40 \mu \mathrm{m}$ with $200 \mu \mathrm{m}$ wide anode pads [4]. Use of such narrow pads in the ILD-TPC would require an excessively large number of readout channels. This complication has been overcome by employing the process of charge dispersion [5] (described in section 2.4.2) first developed by the ILC group at Carleton University. Charge dispersion allows for the use of pads which are an order of magnitude wider, while still achieving the ILD-TPC resolution goals. The process of charge dispersion is achieved by adhering a resistive foil to the anode readout pads with a dielectric glue. A TPC which utilizes this improved readout 
system will henceforth be referred to as a resistive anode MPGD TPC.

\subsection{Motivation}

The large 1 metre prototype TPC (LP-TPC), used in the present study, was constructed in 2008 to develop the design for the much larger ILD-TPC. For the present study the LP-TPC had been equipped with a resistive anode MPGD readout module. The readout electronics used during the beam test of the resistive anode MPGD readout module amplify the pad charge pulse from the front-end preamplifier. The rise time of the front-end preamplifier pulse is larger than its intrinsic rise time due to longitudinal diffusion of the electron cluster, the induction time of the MPGD, and charge dispersion. The amplifier shapes the preamplifier pulse with an adjustable shaping time between 100 and 2000 ns. Existing analysis techniques have shown a shaping time of $500 \mathrm{~ns}$ to achieve the best single hit $\mathrm{r} \phi$ resolution [6]. The $500 \mathrm{~ns}$ shaping time was needed to integrate all electrons contributing to the pad preamplifier pulse in order to achieve resolution limited only by diffusion and electron statistics.

However, long shaping time pulses require significantly more time to return to baseline. Figure 1.3 below shows examples of shaped pad pulses digitized by a FADC (Flash Analog to Digital Converter) for shaping times of 100, 200, 400 and $500 \mathrm{~ns}$. The slow fall times of pulses from long shaping times will degrade the TPC longitudinal resolution as well as its ability to resolve multiple tracks.

In order to concurrently achieve both the single point transverse and longitudinal resolution and prevent signal pile up (resulting in decreased 2 track resolving power), a new FADC pulse analysis technique using shorter shaping times is required. 


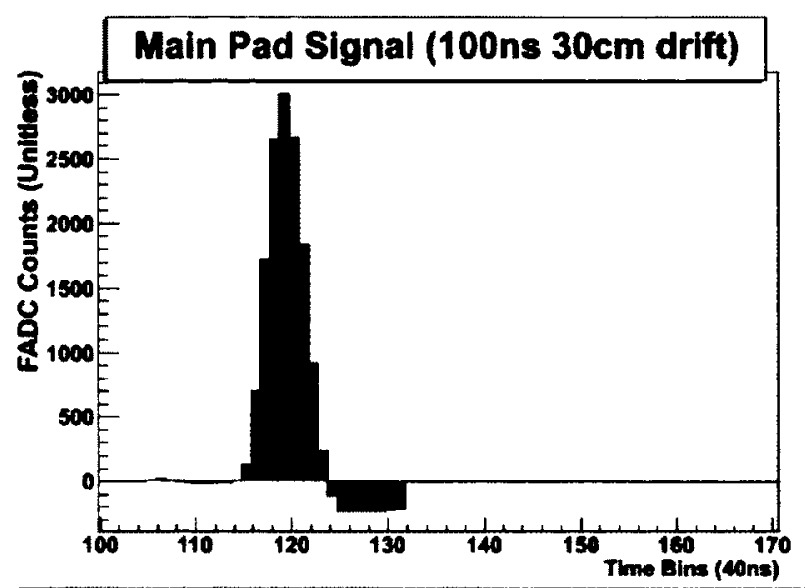

(a)

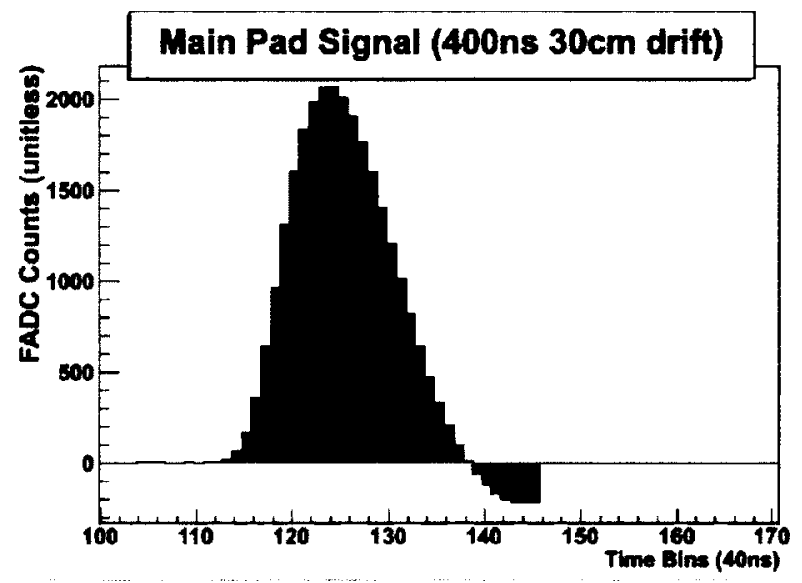

(c)

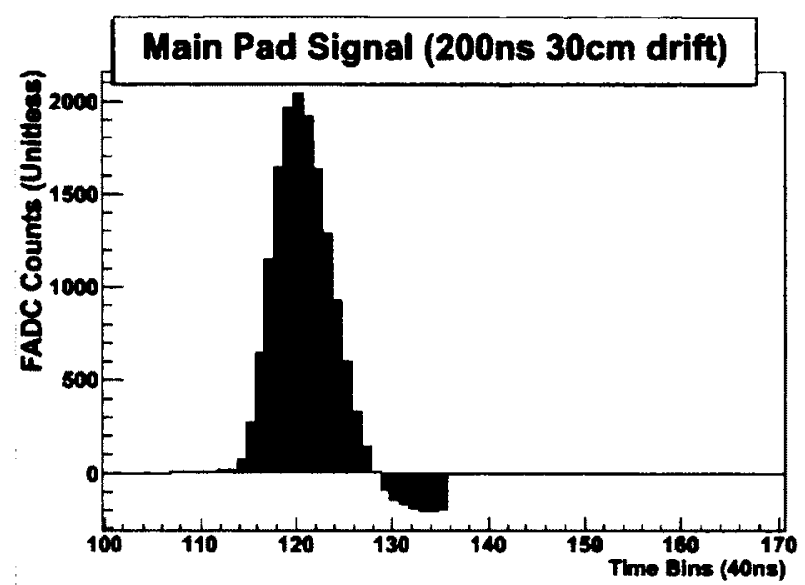

(b)

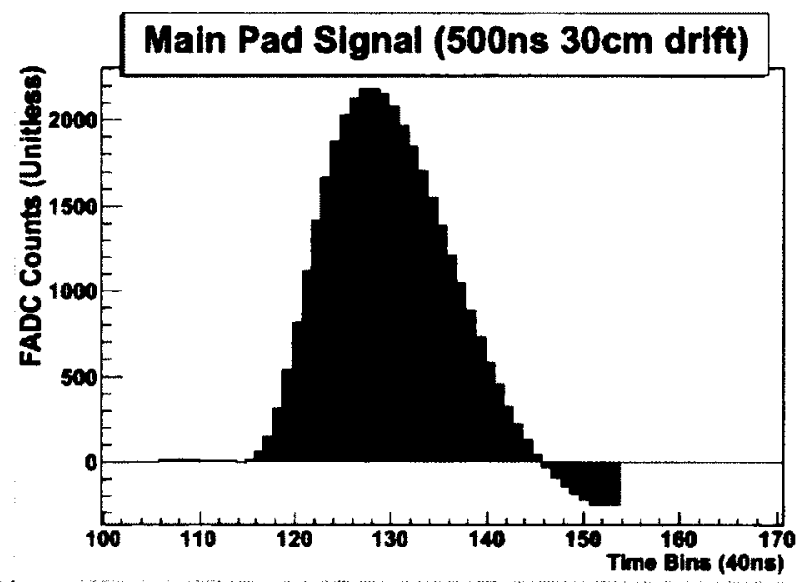

(d)

Figure 1.3: Comparison of FADC pulses for different peaking times. Note the time required for FADC pulses to return to baseline increases with shaping time.

\subsection{Thesis Layout}

This thesis contains the results obtained from the May 2011 beam test of the resistive anode MPGD equipped LP-TPC. The analysis uses two methods for determining the single hit $\mathrm{r} \phi$ resolution. The first method uses existing pulse analysis technique for shaping times between $100 \mathrm{~ns}$ and 500 ns. The second method, developed for this thesis, uses a new pulse analysis technique called the Reintegration Method. Because the shortest possible shaping time is desired, measurements for the Reintegration 
Method were carried out only for $100 \mathrm{~ns}$ data. The results from the different analysis techniques are then compared.

The thesis proceeds as follows:

1. Chapter 2 covers the fundamental physics of a TPC, as well as the workings of a traditional wire TPC readout and its limitations. It then goes on to describe the resistive anode MPGD readout developed by the ILC group at Carleton.

2. Chapter 3 describes the experimental setup used to collect the data analyzed in this thesis. It describes the TPC readout electronics and how the different shaping times affect the detector signals. It also describes the different data sets collected.

3. Chapter 4 explains the steps used in the analysis of the data. The description starts at the analysis of the shaped FADC pulses and ends with the calculation of the resolution measurements.

4. Chapter 5 presents the results of the resolution measurements as well as a comparison of the different techniques. It also contains an extrapolation of the Reintegration Method resolution measurements to ILC conditions.

5. Chapter 6 summarizes the results obtained and compares them to the ILD-TPC resolution requirements. Work which must be completed in the future is also discussed.

\subsection{Authors Contribution}

I contributed to the work presented in this thesis in a number of ways. Firstly, I actively participate at the DESY beam test of the LP-TPC in May 2011. I analyzed the data collected at the beam test for this thesis. While at the beam test I 
assisted in the daily operation of the test. This included monitoring detector, beam, and magnet parameters, changing gas cylinders, and adjusting experimental variables (drift distances, electronic shaping times, and sampling frequencies). Secondly, while at Carleton I worked with another student on improving and "cleaning up" of the analysis code. The improvements to the code included making changes to handle new data types, implementing new pulse analysis techniques, adjusting data cuts, testing new functional forms of the pad response function (PRF), and general maintenance of the code. Thirdly, I was responsible for applying the analysis code to the data collected. I analyzed 30 separate runs which studied the effects of drift distance and shaping time on single hit transverse resolution. This part of the analysis was carried out using existing analysis techniques. My final and most significant contribution to the work presented in this thesis was the development of a new analysis technique called the Reintegration Method. The aim of the Reintegration Method was to improve the transverse resolution of short shaping time data in order to preserve two hit resolving power and longitudinal resolution. This new method was applied to the data first analyzed using the existing analysis techniques. Upon comparison with the existing analysis methods the Reintegration Method was shown to improve transverse resolution at all drift distances while employing shorter shaping times. 


\section{Chapter 2}

\section{Physics of a TPC}

\section{$2.1 \quad$ Fundamentals of a TPC}

A Time Projection Chamber (TPC) is a gas filled particle detector used to study charged particles. It functions as a high speed 3-D camera that images the ionization tracks of charged particles which travel through its gas volume. The characteristics of the track, its radius of curvature (caused by a magnetic field) and amount of ionization, can be used to measure the particle momentum and to identify the particle type. Figure 2.1 shows the basic setup and operation of a TPC. As shown in figure 1.2 , the ILD-TPC will be cylindrically symmetric with the beam pipe passing through its axis. In addition to this symmerty the ILD-TPC will also be symmetric about its central cathode. For this reason figure 2.1 only shows half the TPC when explaining the basic operation of the detector. The important features shown in the diagram are

the gas volume, the axial magnetic $(\vec{B})$ and electric $(\vec{E})$ fields, the central cathode, and the readout plane which is located on the TPC end plate.

The four steps in the operation of the TPC are shown in figure 2.1. Firstly, (a) ionization is deposited in the gas volume by the charged particle. The ionization electrons are then drifted (b) through the gas volume toward the readout plane, located at the TPC end plate, under the combined influence of the magnetic and electric fields. Once at the readout plane an amplification structure (c) amplifies 


\section{TPC Cross Section}

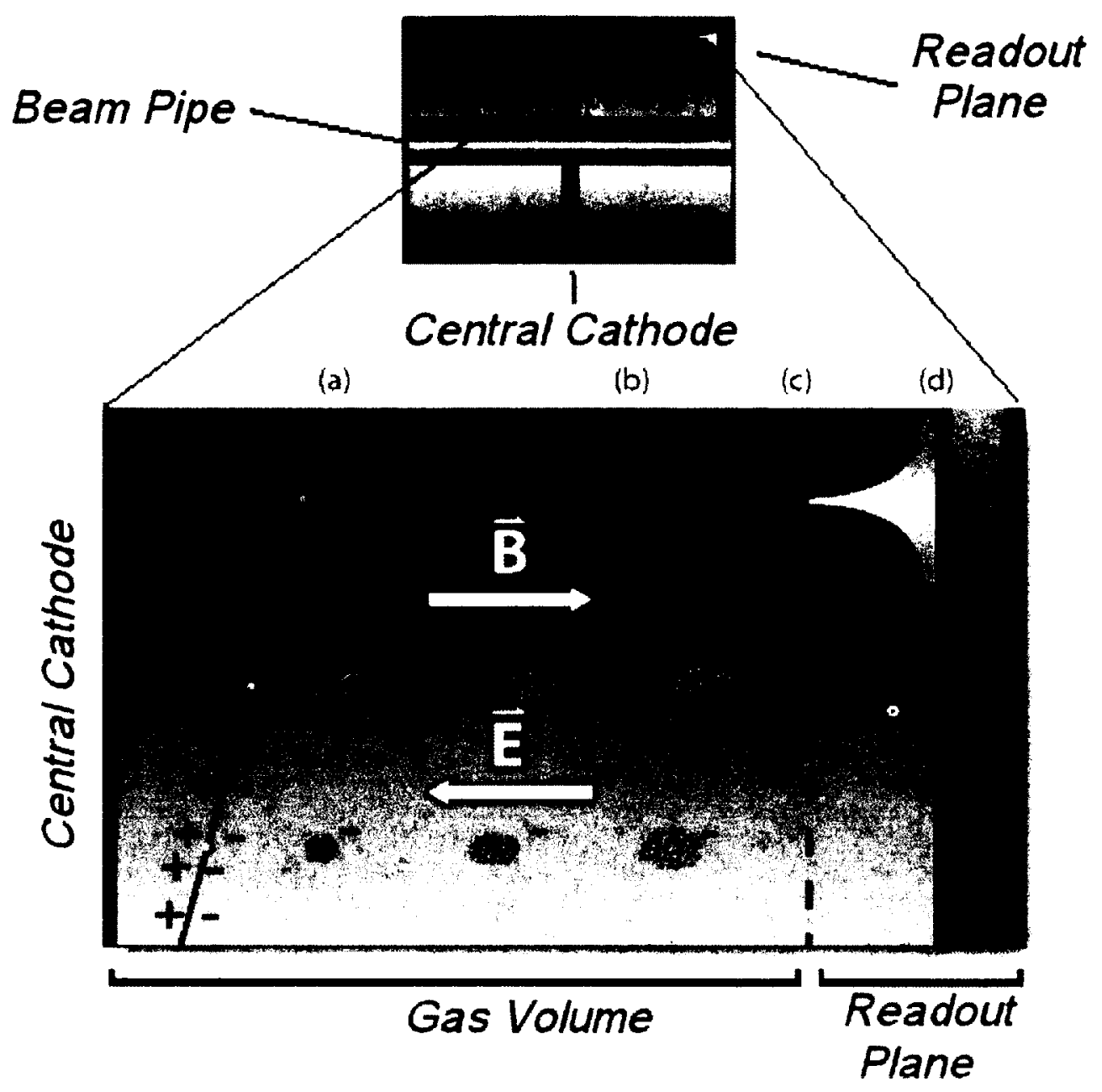

Figure 2.1: Basic setup and operation of a TPC. The smaller diagram shows a cross sectional view of a cylindrical TPC similar to the geometry of the ILDTPC. The beam pipe can be seen running through the TPC. A central cathode separates the TPC into two drift regions. The operation of the TPC is depicted in the zoomed in view of the upper right section: (a) ionization is deposited in the gas volume by the charged particle, (b) ionization electrons drift and diffuse toward the readout plane (ions drift toward cathode), (c) at the readout plane, an amplification structure amplifies the ionization, (d) the charge created in the amplification causes signal in readout pads. A TPC is symmetric about its central cathode so only half the TPC is depicted in this figure. 
the ionization. Lastly, the charge created in the amplification causes signals to be produced in the readout structure (d) on the readout plane.

The first two stages are common to both the traditional wire TPC and the resistive anode MPGD TPC. The fundamentals of ionization and drifting will be discussed in the following section. The amplification and readout of the wire and resistive anode MPGD TPC will then be discussed in following sections.

As a note, the coordinate system shown in figure 2.1 will be used throughout this thesis. The readout plane is located at $z=0$ with the origin of the coordinate system at its center. There are rows of pads extending in the $\mathrm{x}$ direction, and columns of pads in the $y$ direction. The axial magnetic and electric fields are in the positive $\mathrm{z}$ direction. When something is referred to as being transverse or longitudinal it is with respect to these fields. The gas volume extends from the xy plane into the positive z region. The resolutions $\sigma_{r \phi}$ and $\sigma_{r z}$ also called (transverse and longitudinal resolutions, respectively) refer to a cylindrical system based on the symmetry of the TPC. The single hit $\sigma_{r \phi}$ resolution refers to the resolution in the $\mathrm{r} \phi$ plane which is also the xy plane. Likewise, the single hit $\sigma_{r z}$ resolution refers to the resolution in the rz plane which is in the $\mathrm{z}$ direction (into the gas volume).

Although the readout module and readout pads studied in this thesis are keystone shaped (see section 3.1 for description of the detector) and lend themselves well to a cylindrical coordinate system, the Cartesian system described above will be used. There are two reasons for this choice of coordinate system. The first reason is that the software used to analyze the data studied in this thesis was originally written for a TPC with a rectangular readout geometry. The second reason is that the readout pads are nearly rectangular and therefore Cartesian coordinates can still be used to 
simplify some calculations and descriptions.

\subsection{Ionization and Drift}

\subsubsection{Ionization}

As a charged particle traverses the gas volume of a TPC, it interacts electromagnetically with the gas molecules producing clusters of ionization. The size of these clusters (number of electrons) and their frequency along the charged particles path are probabilistic. The spacing between clusters follows a Poisson Distribution with a mean which depends on the electron density in the gas and the cross section for ionization of the gas. The size of a particular cluster is dependent on fluctuations in the average energy deposited per unit length by the charged particle. Although the typical cluster has only a few electrons, the cluster size distribution has a long upper tail caused by rare high energy ionization electrons which cause significant secondary ionization. The cluster size distribution including this long tail is described by a Landau curve [7].

The average energy deposited per unit length, $d E / d x$, is dependent on the particle momentum and its mass. The $d E / d x$ of a particle is well described by the Bethe-Bloch equation [8] (equation 2.1).

$$
\frac{d E}{d x}=\frac{4 \pi N e^{4}}{m_{e} c^{2}} \frac{z^{2}}{\beta^{2}}\left[\ln \left(\frac{2 m c^{2}}{I} \beta^{2} \gamma^{2}\right)-\beta^{2}-\frac{\partial(\beta)}{2}\right]
$$

In equation $2.1 \mathrm{~N}$ and $\mathrm{I}$ are the electron density and mean ionization potential of the absorbing material. In the case of a TPC the absorbing material is the gas. $\beta, \mathrm{z}$, and $\gamma$ are the speed (in terms of c), charge, and Lorentz factor of the incident particle. Lastly, $\partial(\beta)$ is the so-called density effect factor, which accounts for screening of the incident particle electric field by the atoms of the absorber. 
Figure 2.2 shows the theoretical $d E / d x$ over a range of momenta for six particle types calculated using equation 2.1. It also shows the measured $d E / d x$ verses measured momenta for various unknown particles. As the figure shows, this relationship closely follows the theoretical calculations of $d E / d x$. Particles can be identified after their momentum and average energy deposited per unit length have been measured by referring to figure 2.2 .

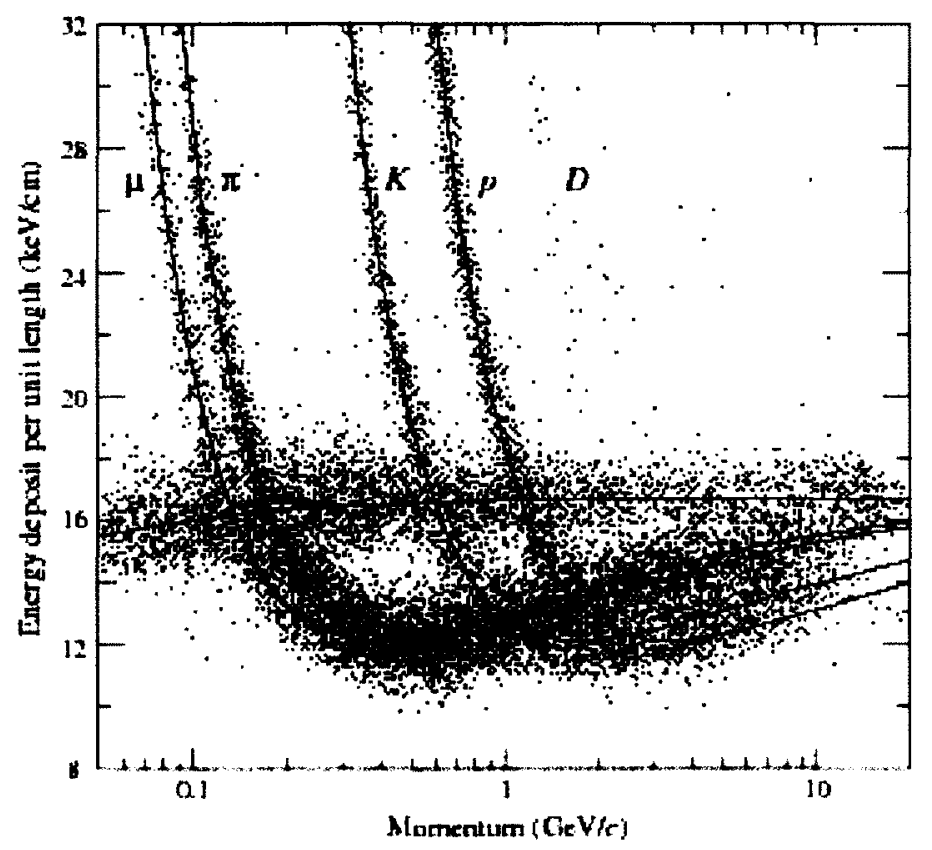

Figure 2.2: Scatter plot of average energy deposited per unit distance verses momentum which can be used to identify a particle. Used with permission of the Particle Data Group [9].

As the charged particle traverses the gas volume, its trajectory is bent due to the magnetic field (shown in figure 2.1). The radius of curvature of the path is inversely proportional to the strength of the magnetic field and proportional the particle momentum (equation 2.2),

$$
R=\frac{|\vec{p}|}{q|\vec{B}|}
$$


Here $\mathrm{R}$ is the radius of curvature, $|\vec{p}|$ is the magnitude of momentum, $\mathrm{q}$ is the charge of the particle, and $|\vec{B}|$ is the magnitude of the magnetic field. If the particle trajectory is reconstructed, then the radius of curvature can be used to measure the particle momentum.

\subsubsection{Drift}

After the ionization electrons have been produced they begin to drift toward the TPC readout plane under the influence of the electric and magnetic fields. The motion of the electrons as they drift is described by 3 parameters; the drift velocity, $\vec{v}_{D r i f t}$, and the coefficients of longitudinal and transverse diffusion $D_{L}$ and $D_{T r}$, respectively. These parameters can be understood by considering a simple model of electron transport.

A drifting electron in a gas has two components to its velocity, as shown in equation 2.3: a thermal component, $\vec{v}_{\text {thermal }}$, and a component resulting from the influence of the electric and magnetic fields, $\vec{v}_{\text {fields }}$.

$$
\vec{v}_{\text {electron }}=\vec{v}_{\text {thermal }}+\vec{v}_{\text {fields }}
$$

The thermal component of the electron's velocity has a randomly oriented direction and a magnitude following a Maxwell distribution. As for the component of the electron's velocity caused by the electric and magnetic fields, $\vec{v}_{\text {fields }}$, since the fields are aligned $\vec{v}_{\text {fields }}$ is in the same direction as the electric field. The electron is accelerated by the electric field between collisions with gas molecules.

Immediately after ionization, the electron has only a thermal component to its velocity. It is then accelerated by the electric field for some amount of time until it scatters off a gas molecule. Upon this interaction the electron loses the preferred directionality of its velocity gained from the fields. The time between scattering 
events follows a Poisson distribution with mean $\tau$. This time interval, $\tau$, is called the mean free flight time.

Averaging the stop start motion of the electron over a time $t>>$ (equivalently over many collisions) the electron's velocity reaches a steady state. This constant velocity is called the drift velocity, $\vec{v}_{\text {Drift }}$. When viewed at the macroscopic scale of the TPC, the electrons drift smoothly toward the readout plane at the constant rate of $\vec{v}_{\text {Drift }}$.

The random motion caused by the thermal component of the velocity results in diffusion of the electrons in the gas. Initially the electrons are located relatively close to the point of ionization (disregarding their relatively slow drift toward the readout plane). However, over time the electron positions become a 3-D Gaussian probability distribution. The standard deviation of the distribution is described by the coefficient of diffusion. The diffusion is not isotropic in a TPC. It is suppressed in the transverse direction by the magnetic field. The dependence of the standard deviations of the distribution of positions in the transverse and longitudinal directions on drift distance are shown in equation 2.4 .

$$
\sigma_{T r, L}=D_{T r, L} \sqrt{z}
$$

Here $\mathrm{z}$ is the drift distance and $D_{T r, L}$ is the coefficient of transverse or longitudinal diffusion.

Usually the coefficient of diffusion is given in units of length $/ \sqrt{\text { time }}$. It is more convenient in the case of a TPC to define the coefficient of diffusion in units of length $/ \sqrt{\text { length}}$, where the $\sqrt{\text { length }}$ is the drift distance. This is because the amount of time an electron has been diffusing for is directly related to the distance the electron has drift. This direct relation is a result of the constant drift velocity, $\vec{v}_{\text {Drift }}$, of the electron. 
Direct calculations of the coefficients of diffusion and drift velocity from the motion of the electrons requires knowledge of the mean free flight time, $\tau$, between collisions with the gas molecules. Values of this parameter are not readily available or easily calculated. However, the software package Magboltz [10] can calculate these parameters using a Monte Carlo simulation of electron transport. Values for the drift parameters, calculated using Magboltz, in the LP-TPC are shown in table 3.1.

It should be noted that the coefficient of transverse diffusion, in table 3.1 , is much smaller then the coefficient of longitudinal diffusion. As mentioned above this is due to the presence of the magnetic field. Any motion transverse to the magnetic field has its displacement suppressed because it is forced to travel along a curved rather than straight line. So after many collisions the total displacement in the transverse direction is suppressed.

The remaining steps in the operation of the TPC, the amplification and readout, differ between the traditional wire and the resistive anode MPGD TPC. These will be discussed in separate sections for each type of TPC.

\subsection{Traditional Wire TPC}

\subsubsection{Wire Amplification}

Traditional TPCs have used an anode wire/cathode pad amplification and readout structure. This setup is illustrated in figure 2.3. Amplification of the electron clusters occurs around the anode wires which are held at a high voltage. As the electrons drift closer to the anode they begin to rapidly gain energy in the high field area near the wire. Eventually the electrons have sufficient energy to begin ionizing the gas molecules and thus creating secondary electrons. These secondary electrons then go through the same process of liberating other electrons from the gas. This process is 
known as an electron avalanche. The avalanche produces sufficient amplification of the original electron cluster to produce a measurable signal on the wire and cathode pads located below the wires.

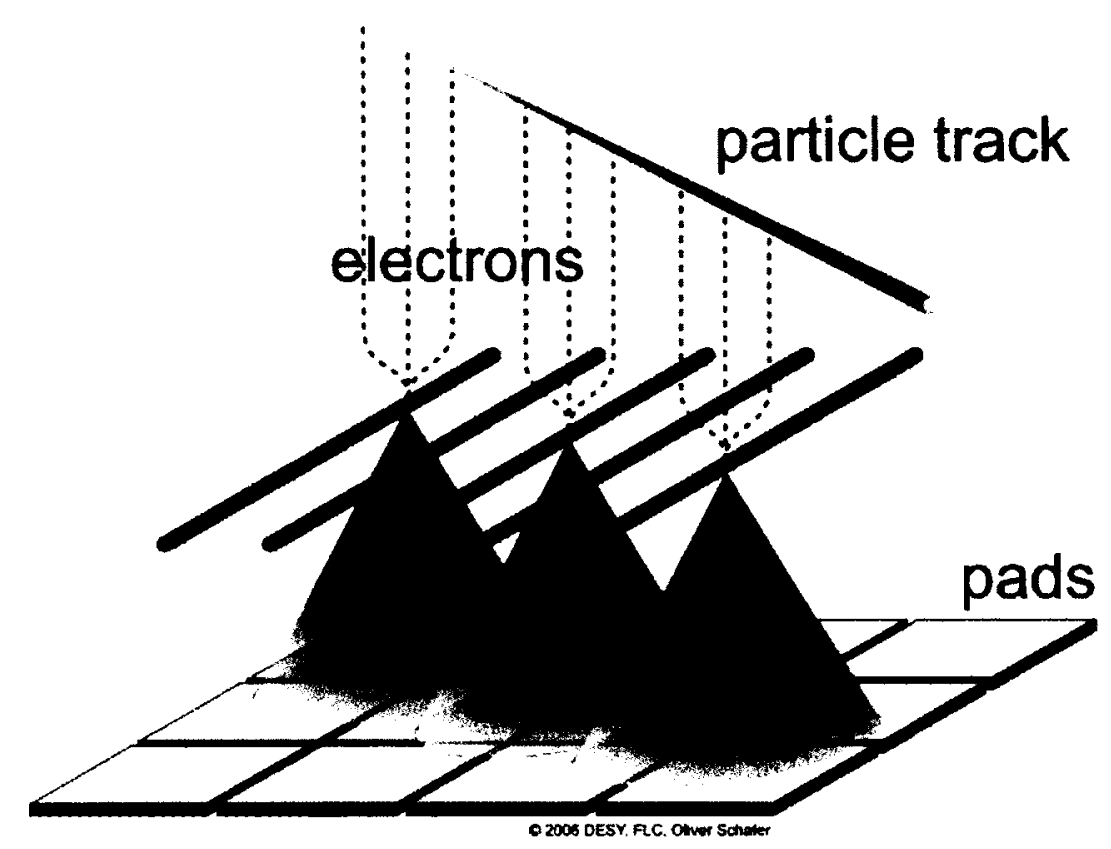

Figure 2.3: Anode wire/cathode pad TPC amplification and readout structure [11]. Used with permission from image creator Oliver Shafer (DESY).

The traditional wire TPC resolution is fundamentally limited by the so called $\vec{E} \times \vec{B}$ effect [12]. The effect is depicted in figure 2.4.

In the drift region the electric and magnetic fields are aligned and so the ionization electrons drift parallel to the magnetic field. However, near the anode wires the electric field becomes radial and bends away from the magnetic field. This causes the electrons to develop a component to their velocity which is transverse to the magnetic field. This causes a force of the form shown in equation 2.5 to move the electrons parallel to the anode wire.

$$
\vec{F}_{B}=q \vec{v}_{d r i f t} \times \vec{B}
$$




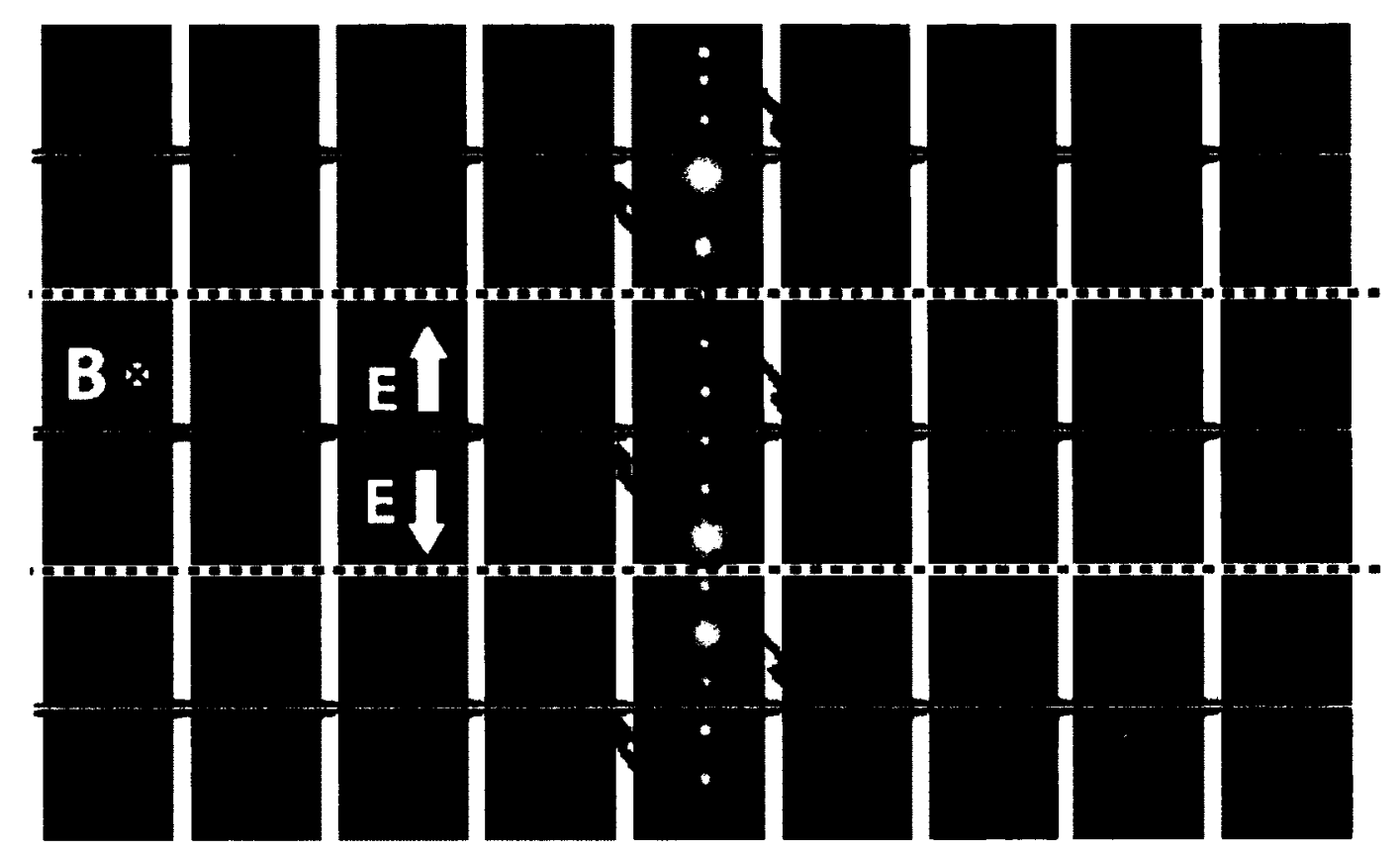

Figure 2.4: The $\vec{E} \times \vec{B}$ Effect. This diagram depicts a view of the readout plane from inside the gas volume of the TPC. The readout pads are shown as blue rectangles, the anode wires can be seen (in gray) stretched out above the rows of readout pads. The clusters of ionization are shown as yellow circles. The different sizes of the circles depicts the differing amount of charge per cluster. The electric field lines near the anode wires are radial to the wire. This causes the electrons to develop a component in their velocity which is transverse to the magnetic field. This results in a rotation of the track segment above each wire. The dashed line separates the segment of the track rotated by the central wire from the segments rotated by the wires above and below.

This results in a rotation of the section of the track above each anode wire. The $\vec{E} \times \vec{B}$ effect would be less of an issue if the electron clusters were all about the same size. However, the cluster size distribution follows a Landau curve so some clusters are significantly larger than the others. The size of the electron cluster affects the amount of charge created in the avalanche. In turn, the readout pad amplitude is proportional to the charge in the electron avalanche. If the clusters were about the same size the weighted mean would reconstruct the correct avalanche position with somewhat larger error. However, since the clusters differ in size significantly, the 
weighted mean cannot accurately reproduce the avalanche position. This is because a larger cluster will skew the weighted mean toward its location.

The wire TPC is also limited by the large number of positive ions created at the anode wire during the avalanche process. The ions disrupt the operation of the TPC in two regions: the amplification region around the anode wires, and the drift region in the gas volume. The positive ions have a mass which is $\sim 2000$ times that of the electrons. Since they are accelerated by the same force the ions travel $\sim 2000$ times

slower than the electrons. For this reason the positive ions can accumulate around the anode wires, and only slowly drift away. This reduces the accelerating potential created by the anode wires reducing the wire gain. Some of the positive ions drift away from the anode wires and follow the electric field lines back to the cathode at the opposite end of the drift region. At high rates, these ions can create a significant space charge which disrupts the electric field in the drift region. Wire TPCs overcome the limitations imposed by the positive ions by using a gating grid.

These effects (mainly $\vec{E} \times \vec{B}$ effect) prevent the traditional wire TPC from achieving the resolution goals of the ILD-TPC. They have spurred the development of new TPC amplification and readout structures.

\subsubsection{Traditional Wire TPC Readout}

The cathode pads in a traditional wire TPC readout are laid out in rows under the anode wires (see figure 2.3). The pads generally have an elongated keystone shape and are aligned with their long axis perpendicular to the anode wires. When an avalanche occurs a capacitive signal is seen by the pads in the underlying pad row. The amplitude of the signal seen by the pad is proportional to the distance from the avalanche. By computing the centroid of pad signals the position of the avalanche along the wire can be determined.

The path of the charged particle is reconstructed from a number of $(x, y, z)$ points. 
The number of points is determined by the number of pad rows. The $\mathrm{x}$ position is calculated from the centroid calculation of the pad signals. The y coordinate is determined from the position of the anode wire which caused the avalanche. The $\mathrm{z}$ coordinate is calculated from the drift time (see equation 2.6). The drift time is defined as the time between the charged particle entering the gas volume, $t_{0}$ (measured using a fast trigger system), and the time when the avalanche occurs, $t_{\text {signal }}$ (measured from the timing of the pad pulses). Multiplying this time by the drift velocity, $v_{d r i f t}$, (which is constant, see section 2.2.2) gives the distance from the readout plane.

$$
z=v_{\text {drift }}\left(t_{0}-t_{\text {signal }}\right)
$$

\subsection{The Resistive Anode MPGD TPC}

\subsubsection{Amplification by MircoMeGaS}

The limitations of the traditional TPC readout discussed in section 2.3 prevent such a TPC from achieving the ILD-TPC resolution requirements. For these reasons a new type of amplification and readout structure is required. Improvements to the TPCs resolution can be achieved by employing so-called Micro Pattern Gas Detectors (MPGDs). Two different types of MPGDs have been developed. The Gas Electron Multiplier (GEM) [13] and the Micro Mesh Gaseous Structure (MicroMeGaS) [14]. Previous studies have demonstrated the ability of GEMs to achieve the ILD-TPC resolution requirements using $1 \mathrm{~mm}$ wide readout pads [15]. However, complications in the operation of GEMs with much larger number of readout pads, and their tendency to damage themselves through sparking at high voltages has lead the ILC group at Carleton to focus on the use of the MicroMeGaS MPGD.

A schematic diagram of how a MicroMegas functions is shown in figure 2.5. The diagram is divided into two regions (labelled a, and b, along the right hand side), 
and each region is bounded by a plane of constant voltage (labelled HV1, HV2, and Anode). Region (a) is the drift region. This is the volume of gas where the charged

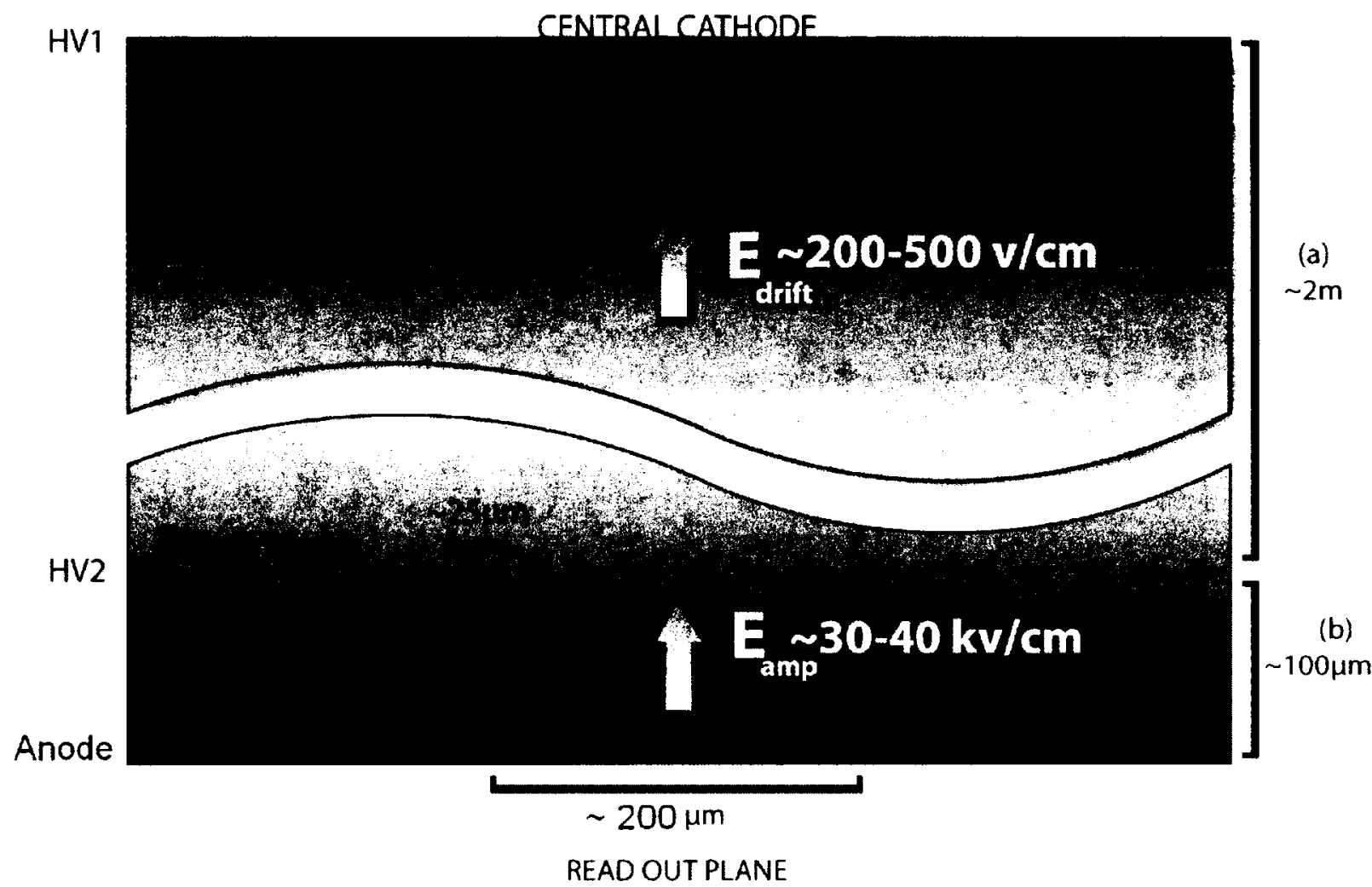

Figure 2.5: Schematic diagram of MicroMegas. (a) represents the drift region and (b) is the amplification region. The MicroMeGas mesh, which separates the drift region from the amplification region, defines a plane of constant voltage labelled HV2. The mesh is supported by insulating pillars not shown in the diagram. The drift field is created by the potential difference between the mesh (HV2) and the central cathode (HV1). the amplification field is created by the potential difference between HV2 and the readout plane (Anode). Due to the proximity of the mesh to the readout plane $(\sim 100 \mu \mathrm{m})$ the electric field, even for modest potential differences, is large.

particle would travel depositing clusters of ionization. These clusters then drift to the amplification region, labelled (b). The drift field is defined by the voltages at HV1 and HV2. These voltages can be set to create a suitable drift field of a few hundred $\mathrm{V} / \mathrm{cm}$. In figure 2.5 the amplification region is divided from the drift region by a dashed line. This line represents the MicroMeGaS mesh which is a fine metallic mesh 
with center to center spacing of $\sim 25 \mu \mathrm{m}$. The fine spacing of the holes ensures that the distortion of the electron trajectory is minimized. The spacing between the mesh and the readout plane, region (b), is $\sim 100 \mu \mathrm{m}$, this is called the amplification region. The mesh is supported above the readout plane by insulating spacers called pillars (not shown). The spacing between pillars is $\sim 2 \mathrm{~mm}$ ensuring only a few stand above any one readout pad, preventing distortion of the amplification field. The small gap between the planes HV2 and Anode produces the amplification field. These voltages can be adjusted to produce a field of $30-40 \mathrm{kV} / \mathrm{cm}$.

The MicroMeGaS amplification structure deals with both factors which limit the traditional anode wire TPC. Firstly, the resolution of the traditional TPC was limited by the $\vec{E} \times \vec{B}$ effect. The MicroMeGaS mesh essentially does not alter the direction of the electric field thus keeping the electric and magnetic fields well aligned. This minimizes the amount of transverse motion by the electrons and thus effectively removes the $\vec{E} \times \vec{B}$ effect. Secondly, the amount of slow moving positive ions created by the anode wires of the traditional TPC, could reduce the amplification field of the anode wires and disrupt the electric field in the drift region. The MicroMeGaS mesh creates a barrier to the positive ions which efficiently collects the ions and prevents them from entering the drift region. The high electric field and small distance between the ions and the mesh quickly removes the ions from the amplification region. This prevents a build up of positive charge in the amplification region which would reduce amplification, as was the case in the traditional wire TPC.

\subsubsection{Charge Dispersion Readout}

The LP-TPC readout module utilizes MicroMeGaS to amplify the electron clusters. However, due to the proximity of the amplification structure to the readout pads the resulting electron avalanche is very localized. In previous applications of the MicroMeGaS this had not been an issue because they had been used to instrument 
relatively small areas. Instrumenting small areas meant narrow ( $\sim 200 \mu \mathrm{m})$ pads could be used without the large number of readout channels required becoming an issue. However, the ILD-TPC requires an area of $\sim 10 \mathrm{~m}^{2}$ to be instrumented. An area of this size would require an unmanageable number of readout channels if such narrow pads were used. To overcome this issue the phenomenon of charge dispersion is used.

Charge dispersion was first developed and studied by the ILC group at Carleton University [16]. The process is used to disperse avalanche charge over several anode pads of the MPGD TPC readout system. The charge dispersion is achieved by forming a two dimensional resistive-capacitive network on the readout plane.

To produce the $2 \mathrm{D} \mathrm{RC}$ network a high resistivity (on the order of a few $\mathrm{M} \Omega / \square$ ) foil is adhered to the readout plane with an insulating glue. Figure 2.6 shows a cross sectional view of the resistive anode. Any charge which is deposited on to this foil disperses with an RC time constant much like the charge on a capacitor in an $\mathrm{RC}$ circuit. The RC time constant is defined by the resistivity of the foil, and the capacitance density between the foil and the readout plane.

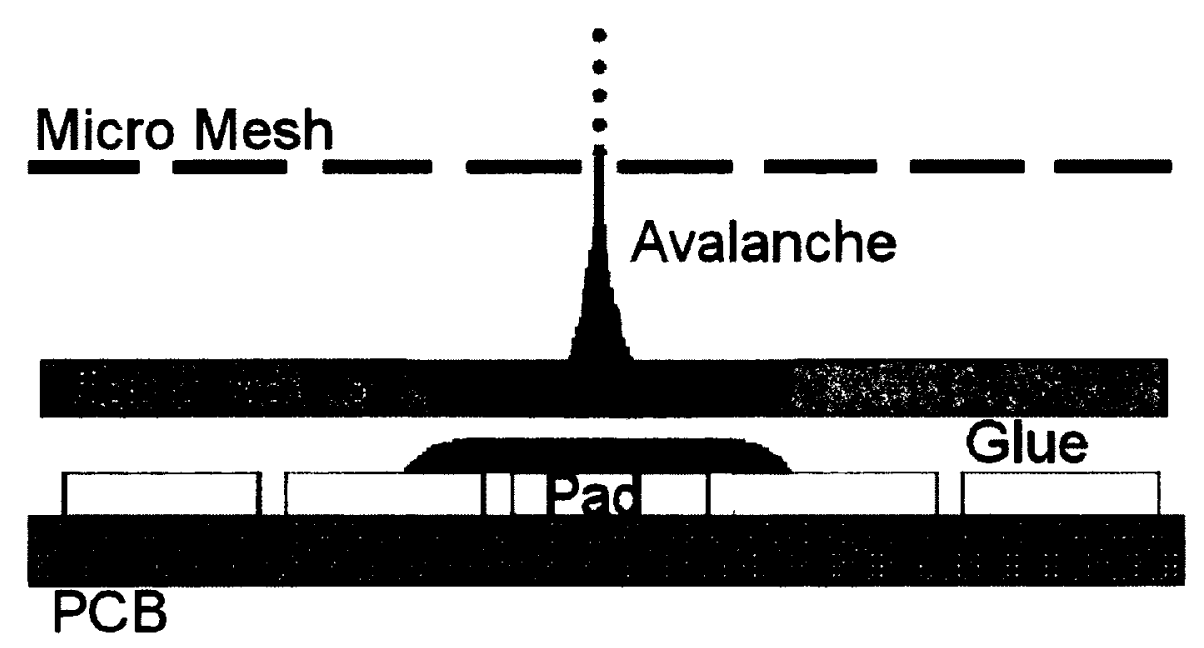

Figure 2.6: Cross sectional view of the resistive anode which allows for charge dispersion on the readout plane. The charge from the MicroMeGas avalanche can be seen dispersing across the surface of the resistive foil. 
The dispersion of the charge on the foil is modelled by the $2 \mathrm{D}$ Telegraph equation shown in equation 2.7 .

$$
\frac{\partial \rho}{\partial t}=\frac{1}{R C}\left[\frac{\partial^{2} \rho}{\partial r^{2}}+\frac{1}{r} \frac{\partial \rho}{\partial r}\right]
$$

In equation $2.7, \rho(r, t)$ is the surface charge density at a given location and time, $\mathrm{R}$ is the resistivity of the foil, and $\mathrm{C}$ is the capacitance density. The solution for a point charge deposited at the origin is shown in equation 2.8 .

$$
\rho(r, t)=\frac{1}{2 t h} \exp \left(\frac{-r^{2}}{4 t h}\right)
$$

Here $h=1 / R C$ is the inverse of the time constant per unit area of the RC network, and $\mathrm{R}$ and $\mathrm{C}$ are the same as in equation 2.7. The raw signal induced on a pad can be calculated by integrating the time dependent charge density function over the pad area.

The time/space evolution of a point charge and the induced signal on 3 nearby pads is shown in figure 2.7. A simulation of the charge dispersion phenomena in a TPC with MPGD including the effects of diffusion, MPDG rise time, and electronic timing effects has shown good agreement between the model and measurements [17]. 

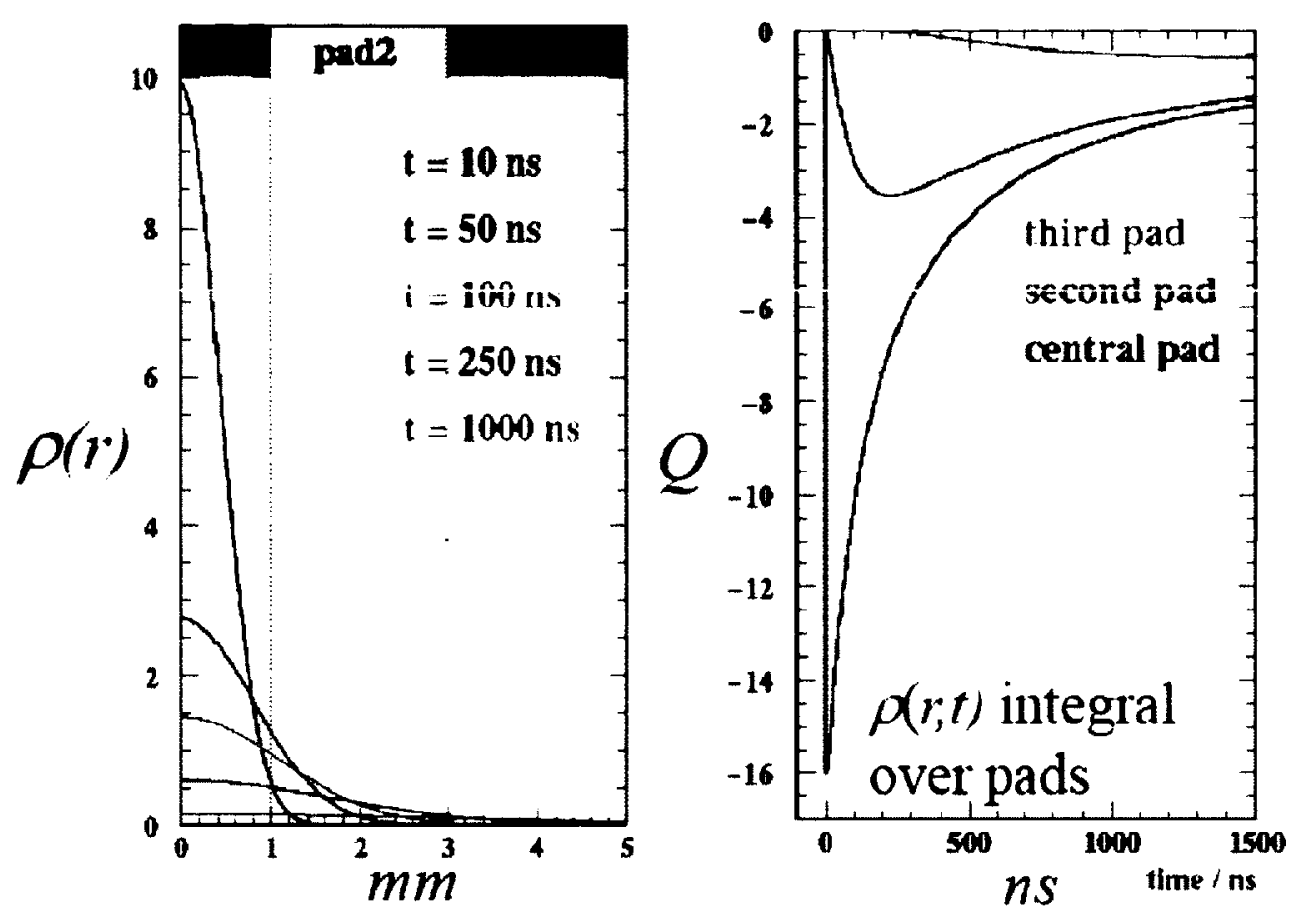

Figure 2.7: Surface charge density and the resultant raw charge pulses induced on neighbouring pads [17]. The distribution of surface charge density is shown on the left at five different times between $10 \mathrm{~ns}$ and $1000 \mathrm{~ns}$. The figure on the right shows the time dependence of the charge signal seen by 3 different pads. The central pad has observed the charge avalanche directly, whereas the second and thirds pads have seen only the charge dispersion signals. 


\section{Chapter 3}

\section{Experimental Setup and Data Taking}

\subsection{Experimental Setup}

The data analyzed in this thesis were collected during a beam test at the DESY laboratory in Hamburg, Germany in May of 2011. The purpose of the beam test was to measure the $\mathrm{z}$ (drift distance) dependence of the resistive anode MPGD TPC's single hit transverse resolution under different settings of the readout electronics (see section 3.2). The experimental hall was setup as shown in figure 3.1. The main components of the setup shown in the figure are,

1. The LP-TPC

2. The superconducting magnet (PCMAG)

3. Moveable stage

4. Electron beam $(5 \mathrm{GeV})$

5. Readout module

6. Trigger System

This section will describe the important features of each component. 


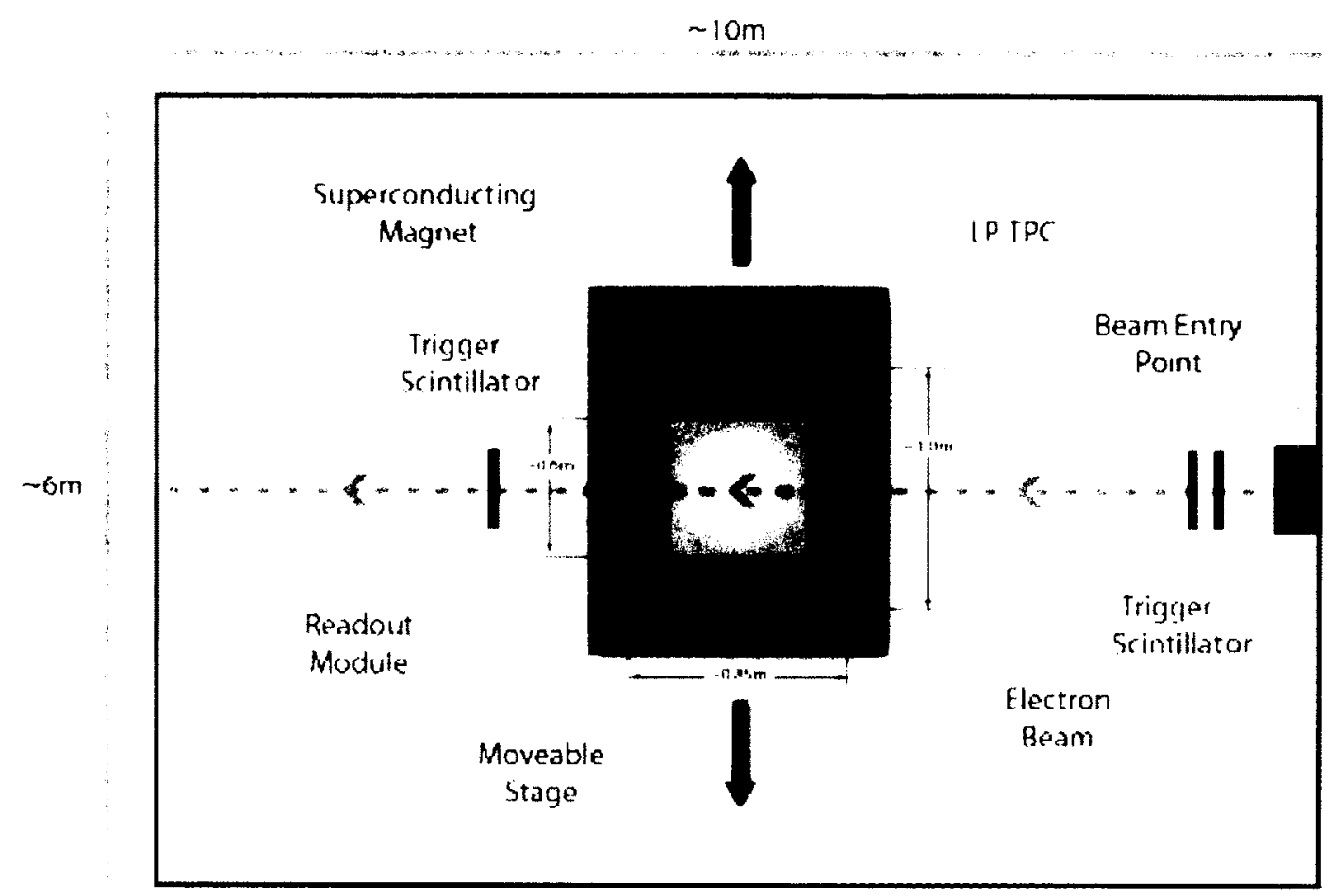

Figure 3.1: Diagram of the experimental setup. The 6 main components of the apparatus can be seen. The motion of the movable stage is shown by the thick arrows.

The LP-TPC (side view shown in figure 3.2) is a cylindrical TPC with a diameter of $\sim 80 \mathrm{~cm}$, and a maximum drift length of $60 \mathrm{~cm}$. The end plate contains 7 keystone shaped ports (see in figure 3.3), arranged in 3 rows, used for testing different readout modules. Only the central port was used during the present test. The drift volume was filled with a gas mixture of $\mathrm{Ar} / C F_{4} / C_{4} H_{10}$ with mixture ratios 95:3:2. The conditions inside the drift volume were kept at standard temperature and a slight over pressure to minimize gas contamination. The drift field was set to $230 \mathrm{~V} / \mathrm{cm}$ and kept constant throughout the tests. The TPC was placed inside a super conducting magnet.

The magnet, called PCMAG [18], has an inner bore radius of $85 \mathrm{~cm}$ and an active length of $1 \mathrm{~m}$. It is capable of supplying up to a $1.2 \mathrm{~T}$ field, though for the purposes of our tests the field was kept at $1 \mathrm{~T}$. The magnetic field produced has been accurately 


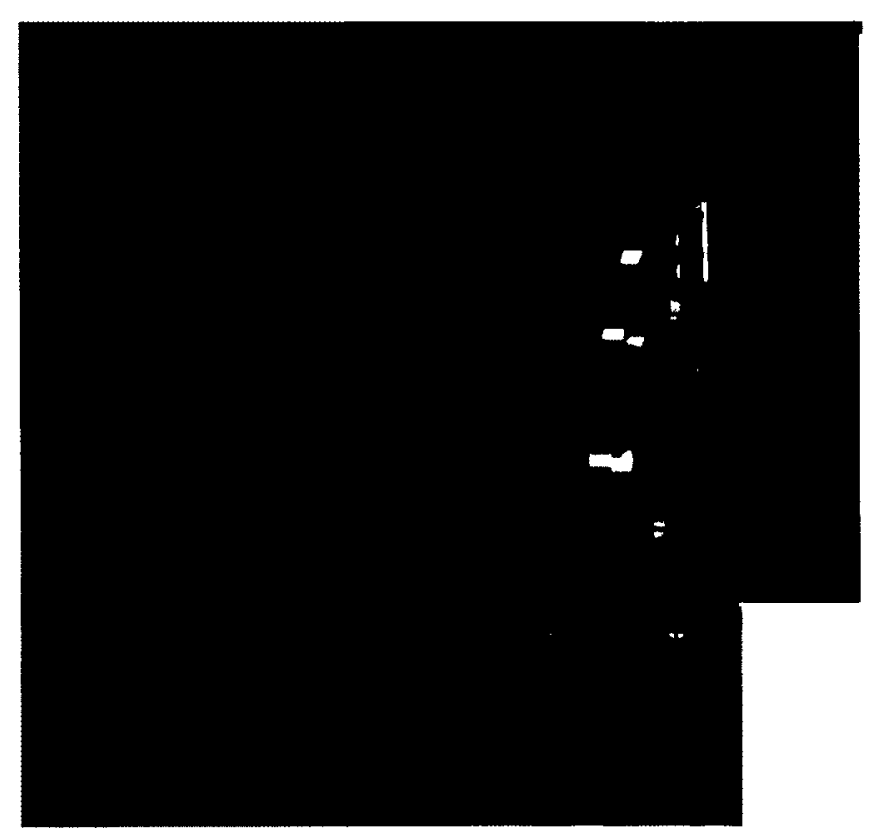

Figure 3.2: Side view of the LP-TPC. Note that an older style of readout electronics is attached to the TPC. The new electronics are integrated into the readout module.

measured and even though it contains no iron it has been shown to have a fairly uniform field in the central region [18]. The LP-TPC was placed close to the center where the field was most homogeneous. The magnet and TPC were mounted on the movable stage.

The movable stage allowed the LP-TPC to be kept at the center of the magnet to ensure a homogenous field but still allowed the location of the beam to be varied relative to the readout plane. The stage is capable of moving perpendicular to the beam axis both vertically and horizontally. However, since the resolution as a function of drift distance was being measured the stage was only used to move the magnet and LP-TPC horizontally in a direction perpendicular to the beam axis (direction of motion shown in figure 3.1).

A $5 \mathrm{GeV}$ electron beam with a spread in momentum of $\Delta p / p \sim 5 \%$, in the T22 DESY test beam area [11], was used in the resolution studies. The TPC was 


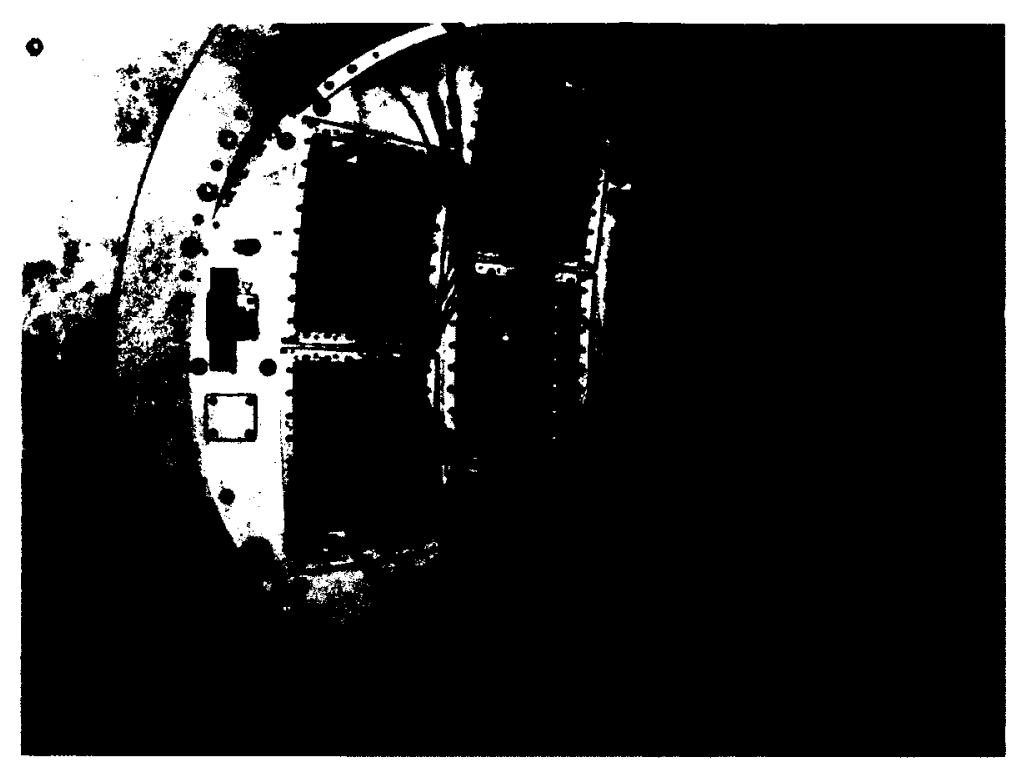

Figure 3.3: Front view of the LP-TPC. The LP-TPC is inside the magnet and rotated so the beam particles travelled from the inner radius to the outer radius of the readout module.

positioned so that the beam would illuminate the central columns of the readout module.

The pad pattern of the readout module tested is shown in figure 3.4. Both the module and the 1728 readout pads are keystone shaped. These shapes were used to mimic a pad layout similar to what will be used in the ILD-TPC. The pads are arranged into 72 columns and 24 rows. They have the same height of $6.28 \mathrm{~mm}$ but vary in width with the bottom row being $2.7 \mathrm{~mm}$ and the top being $3.2 \mathrm{~mm}$ (each pad has a constant angular width of 1.9 mRads). To produce the $2 \mathrm{D} \mathrm{RC}$ network a Kapton foil with resistivity of $2.9 \mathrm{M} \Omega / \square$ was glued to the surface of the pads. The glue thickness was $\sim 75 \mu \mathrm{m}$ and had a dielectric constant of $\sim 4.5$. This leads to a capacitance density of $0.254 \mathrm{pF} / \mathrm{mm}^{2}$. These lead to an RC time constant on the order of $1700 \mathrm{~ns} / \mathrm{mm}^{2}$. The values quoted here are taken from [19]. Due to manufacturing imperfections there are position dependent variations in these values. 


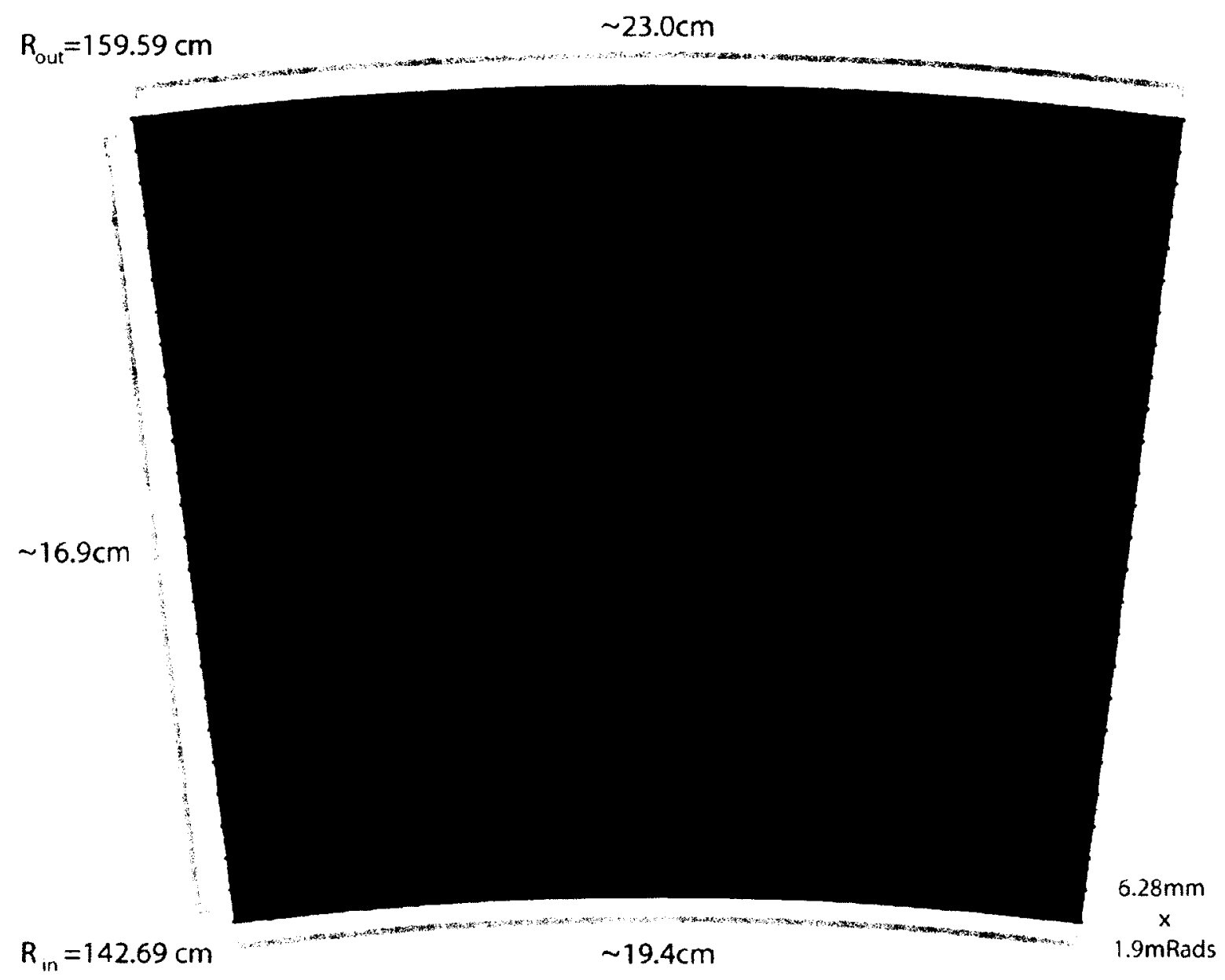

Figure 3.4: Front view of the readout module. The 1728 readout pads are laid out in a keystone geometry. The pads are arranged into 24 rows each with 72 columns. Due to their keystone shape, pads in the bottom rows are narrower $(2.7 \mathrm{~mm})$ than the pads in the top rows $(3,2 \mathrm{~mm})$. The pads outlined in orange (those between rows 12 and 23 and columns 30 and 45) were used in the resolution calculation. Initial analysis of the data showed the other pads were not functioning properly.

These variations result in position dependent systematic biases in track position determination. These bias errors can be calibrated and are corrected for in the analysis of the data (see section 4.3.3). The MicroMeGaS amplification structure is attached above the resistive foil. The MicroMeGaS pillars have a height of $128 \mu \mathrm{m}$ and spacing of $2 \mathrm{~mm}$. The hole to hole spacing is $63 \mu \mathrm{m}$. The gain voltage applied to the mesh was $380 \mathrm{~V}$ giving an amplification field of $\sim 29.6 \mathrm{kV} / \mathrm{cm}$. The readout electronics are 
integrated into the readout module and were located outside of the TPC gas volume. The electronics will be discussed in the next section.

The trigger system shown in the figure 3.1 was used to determine when a beam electron had traversed the TPC gas volume. Three scintillation counters with attached photomultipliers were used to detect the electrons. Two scintillation counters were located upstream of the TPC and the third was placed downstream. If all three counters measured a signal, then a signal was sent to the readout electronics to record data for a sent time window.

Table 3.1 summarizes the experimental parameters which were kept constant throughout the beam test.

\begin{tabular}{|c|c|c|}
\hline Parameter Name & Value & Units \\
\hline Drift Field & 230 & $V / \mathrm{cm}$ \\
\hline Magnetic Field & 1.0 & $\mathrm{~T}$ \\
\hline MicroMeGas Voltage & 380 & $V$ \\
\hline Gas Parameters & & \\
\hline Coefficient of Transverse Diffusion $D_{T r}$ & 94.2 & $\mu m / \sqrt{\mathrm{cm}}$ \\
\hline Coefficient of Longitudinal Diffusion $D_{L}$ & 226.6 & $\mu \mathrm{m} / \sqrt{\mathrm{cm}}$ \\
\hline Drift Velocity $v_{\text {drift }}$ & 76.05 & $\mu \mathrm{m} / \mathrm{ns}$ \\
\hline Beam Parameters & & \\
\hline Mean Momentum & 5.0 & $\mathrm{GeV} / \mathrm{c}$ \\
\hline$\Delta p / p$ & 5 & $\%$ \\
\hline
\end{tabular}

Table 3.1: Summary of Experimental Setup Parameters. 


\subsection{Readout Electronics}

The first studies of a previous resistive anode MPGD TPC undertaken by the ILC group at Carleton were conducted without shaping the preamplifier pulses [20], [21]. Instead the preamplifier pulses were digitized directly using $200 \mathrm{MHz}$ FADCs (Flash Analog to Digital Converter). The tests were conducted in this way to study and understand the new charge dispersion phenomena and to develop techniques to use it for position determination. Using these $200 \mathrm{MHz}$ FADCs would not have been feasible at the ILD-TPC due to their cost and the amount of data which would be produced. Alternatively, a new suite of readout electronics using $25 \mathrm{MHz}$ FADCs could have been developed. However, due to monetary, manpower and time constraints such a system was not be developed. In the end the decision was made to adopt the read out electronics developed for the Tokai to Kamioka (T2K) TPCs [22]. The T2K TPC electronics utilize the so called AFTER chip [22] readout system developed by CEA in Saclay, France. The AFTER chip fully integrates the electronics to the readout module. The new fully integrated electronics contains: front end pre-amp, shaper, a switched capacitor array to store signals on board, multiplexers/ digitizers, zero suppression, FADC, and readout drivers. The final digitized data from the module is readout by a single optical fibre.

$$
H(t)=A_{0}\left(\frac{t}{\tau}\right)^{3} \sin \left(\frac{t}{b \tau}\right) \exp \left(-\frac{t}{\tau}\right)
$$

The main amplifier shaper of readout systems contains an adjustable shaping time. The shaper response to an impulse charge has been experimentally measured and parametrized by the equation 3.1 [23]. Table 3.2 shows parameters for the response function for different shaping times. Figure 3.5 shows the different response functions.

Figure 3.6 shows the readout electronics pipeline. The pipeline is made up of four steps: a raw charge pulse on a pad is amplified in the preamplifier, the preamplifier 


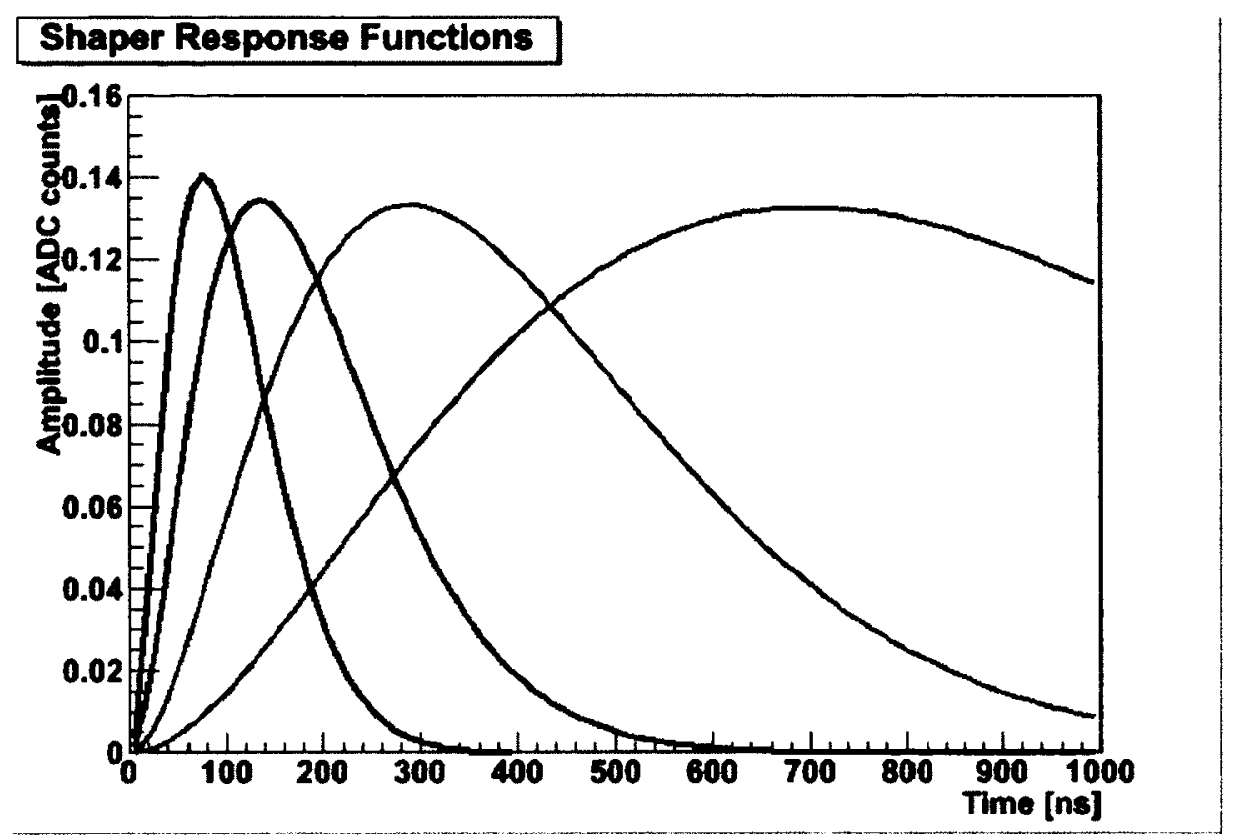

Figure 3.5: Examples of the shaper response functions for a range of different shaping times. (black $100 \mathrm{~ns}$, blue $200 \mathrm{~ns}$, red $400 \mathrm{~ns}$, green $1000 \mathrm{~ns}$ )

pulse is then sent to the shaper amplifier, the shaped pulse is digitized by a FADC (at either 25 or $50 \mathrm{MHz}$ ), and finally the digitized pulse is stored in memory. The affect of the pulse shaping on resolution is studied in this thesis.

\begin{tabular}{|c|c|c|c|}
\hline Shaping Time (ns) & $A_{0}$ & $\mathrm{~b}$ & $\tau(\mathrm{ns})$ \\
\hline 100 & 0.835065 & 3 & $2.05 \times 20$ \\
\hline 200 & 0.91629 & 3.5 & $3.55 \times 20$ \\
\hline 400 & 0.95396 & 3.7 & $7.55 \times 20$ \\
\hline 1000 & 0.984186 & 3.85 & $18.2 \times 20$ \\
\hline
\end{tabular}

Table 3.2: Summary of response function parameters for different shaping times. 


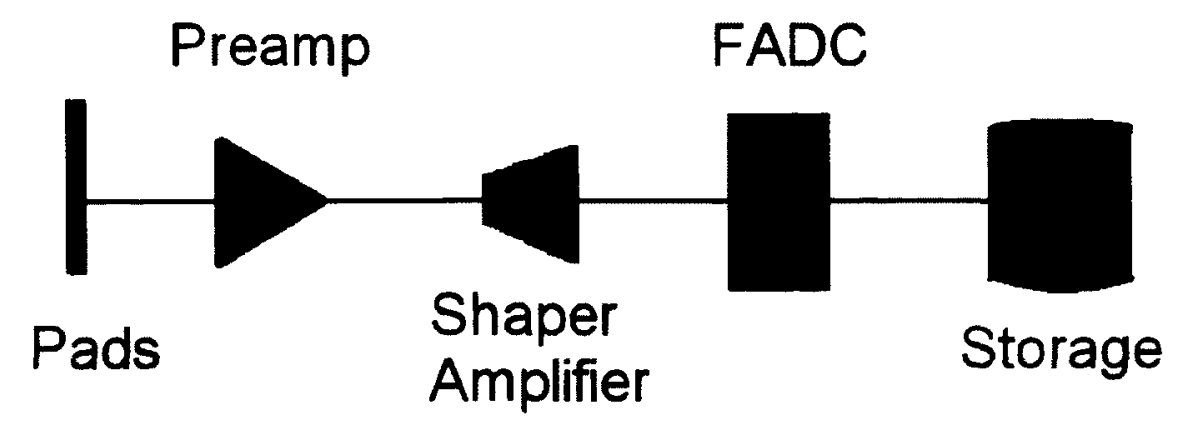

Figure 3.6: Signal readout pipeline.

\subsection{Data Taking}

The data studied in this thesis were collected in 5 scans. Each scan is made up of 6 runs, which are collections of 5000 events taken at fixed drift distances $(10,20,30$, $40,50,55 \mathrm{~cm})$. Each scan studied a different combination of detector settings. The detector settings which were varied between scans were shaping time $(100,200,400$, $500 \mathrm{~ns}$ ), and whether to turn on or off the zero suppression process. These detector settings all refer to the readout electronics. The shaping time characterized the pulse shaping electronics, and zero suppression, a method to automatically record only nonzero data to limit data set sizes, could be turned on or off. All other detector settings were kept constant between scans. These included TPC drift field, MicroMeGas voltage, and magnetic field strengths, as well as gas composition pressure and temperature. Table 3.3 shows an example of scan settings and the runs which are contained in it. A complete list of scans is given in appendix B.

Data were collected for all readout pads during the beam test. Initial analysis of the data showed a number of sections of the readout pad array were not functioning properly. The readout module being tested was the first of a new design to be built. This may account for the improper functioning of parts of the readout. Due to malfunctioning sections, only the pads outlined in orange in figure 3.4 of the readout array were used in the analysis. The functional pads included those in between rows 


\begin{tabular}{|c|c|c|c|c|c|}
\hline \multicolumn{3}{|c|}{ Scan Parameters } & \multicolumn{2}{c|}{ Run Parameters } \\
\hline Scan \# & Shaping Time [ns] & Zero Sup. & Sampling [MHz] & Run \# & $\mathrm{z}_{\text {drift }}[\mathrm{cm}]$ \\
\hline \multirow{4}{*}{1} & & & & 1218 & 10 \\
& & & & 1225 & 20 \\
& \multirow{3}{*}{500} & \multirow{3}{*}{ TRUE } & \multirow{2}{*}{25} & 1230 & 30 \\
& & & & 1235 & 40 \\
& & & & 1240 & 50 \\
& & & & 1245 & 55 \\
\hline
\end{tabular}

Table 3.3: An example of the detector settings used in a scan and the run settings (scan 1).

12 and 23 and between columns 30 and 45 (the pads in other columns may have worked but we used only the pads in the beam region). Of the pads used in the analysis not all were used in the calculation of the resolution. The top and bottom pad rows were not included in the resolution calculation. These rows were omitted due to the method used to calculate the resolution measurement (see section 4.4).

The data in scan 5 were collected with zero suppression turned off. This was done because the runs in this scan were analyzed using a new pulse analysis technique. The zero suppression process affects the recorded pad pulses which would impact the new analysis technique. It truncates the pad pulses below a certain lower limit. Charge dispersion pulses generally have slowly rising pulses with low amplitudes in their leading edge. These time bins, which need to be integrated, would have been removed by the zero suppression process. The new analysis technique depends on the full shape of the pulses, including time bins with negative values, and low amplitude leading edge time bins. For these reasons the zero suppression process had to be turned off. 


\section{Chapter 4}

\section{Analysis}

\subsection{Overview of Analysis}

The purpose of the analysis is to determine the dependence of the detector's single hit $\mathbf{r} \phi$ resolution on drift distance. The process of determining this dependence has four steps. Firstly, the signals from the pads must be analyzed and amplitudes determined. Secondly, these amplitudes are used to determine the trajectory of the charged particle which traversed the TPC. This is called the track fit. Thirdly, systematic biases in position determination resulting from imperfections in the detector assembly must be measured and corrected. And fourthly, after all these steps are taken the resolution can be measured.

The trajectory of the charged particle which traverses the TPC is reconstructed from $\mathrm{n}(\mathrm{x}, \mathrm{y}, \mathrm{z})$ points, where $\mathrm{n}$ is the number of pad rows. Determination of the $\mathrm{y}$ and $\mathrm{z}$ coordinates is the same between the traditional wire and resistive anode MPGD TPC (see section 2.3.2). The $\mathrm{z}$ coordinate is measured from the timing of the signals and the $y$ coordinate is measured from the position of the pad row. However, determination of the $\mathrm{x}$ coordinate differs.

In the case of a traditional wire TPC the preamplifier pulse has the same shape

for each pad. The only difference is the pulse height which is proportional to the 
distance to the track. The $\mathrm{x}$ coordinate can simply be measured by calculating a weighted mean of the pad amplitudes.

In the case of a resistive anode MPGD TPC the entire pulse shape is dependent on the track position. This is due to the charge dispersion. The pulse height, rise time and fall time all differ between pads. Pads which directly observe the charge have larger pulse heights and faster rise and fall times than pads which only see the charge dispersion signal. For this reason a simple weighted mean of the pulse heights will not accurately reconstruct the position of the track (the $\mathrm{x}$ coordinate). Instead a new definition of the pad amplitude is required. Once this new amplitude is defined its dependence on track position can be characterized by a Pad Response Function (PRF). This gives us some freedom in defining the amplitude. The definition of the pad amplitudes used in this thesis are discussed in section 4.3.1.

The PRF relates the distance between a pad center and a track, to the pad amplitude. Since we are free to define the pad amplitude, the PRF can take many forms, and all that is needed is a suitable function to parameterize it. In the present study the PRF has been parameterized by the so-called Product-Form equation,

$$
\operatorname{PRF}(x)=\frac{\exp \left[-4 \ln (2)(1-r) x^{2} / w^{2}\right]}{1+4 r x^{2} / w^{2}}
$$

Equation 4.1 is the product of a Gaussian with a Lorentzian function. The amount of mixing is defined by the parameter $\mathrm{r}$. When $\mathrm{r}$ is 1 the function is fully Lorentzian. When $r$ is 0 the function is fully Gaussian. Both the Lorentzian and the Gaussian share the same width parameter $\mathrm{w}$.

Both PRF parameters, $r$ and $w$, depend on the drift distance of the track, and the shaping time of the readout electronics. In order to use the PRF to determine the $\mathrm{x}$ coordinate it must first be calibrated to the drift distance of the run and shaping time of the scan. The method used to calibrate the PRF parameterization will be 
discussed later in section 4.3.2. Once the PRF has been calibrated it can be used to fit tracks to the observed pad amplitudes. How the track fitting is accomplished will be discussed in section 4.2 .

Local inhomogeneities in the construction of the resistive anode assembly introduce position dependent systematic biases in measurement of the track position. These biases must be measured and corrected for before the resolution can be calculated. Measurements of the inhomogeneities and calculation of the bias correction are conducted during the analysis. How this is done is discussed in section 4.3.3.

The resolution of the detector is determined from the width of a Gaussian fit to the global (meaning from all rows) row residual distribution. A row residual is the difference between the true track position and the fitted track position for the row. However, because no external reference of the track is available the residuals must be estimated using equation 4.2 (see reference [24] for a derivation and justification of this equation).

$$
R=\sqrt{\sigma_{i n} \sigma_{e x}}
$$

Here $\sigma_{i n}$ is called the inclusive track fit residual and $\sigma_{e x}$ is called the exclusive track fit residual. The definition of the inclusive and exclusive residuals and how they are calculated is given in section 4.3.3. An example resolution calculation is shown in section 4.4 .

\subsection{Track Fitting}

Figure 4.1 shows an example of a track fit to an event, and the coordinate system used during the track fitting. The origin of the coordinate system is located in the middle of the readout plane (between the $12^{\text {th }}$ and $13^{\text {th }}$ row, and the $36^{\text {th }}$ and $37^{\text {th }}$ columns). Current track fitting procedures fit curved tracks to the data. The track is described by three parameters, $\left(x_{0}, \phi, 1 / R\right)$, shown in figure 4.1. In figure $4.1 x_{0}$ is 
the $\mathrm{x}$ intercept, $\phi$ is the local azimuthal angle tangent to the track where it crosses the $\mathrm{x}$-axis, and $\mathrm{R}$ is the radius of curvature.

The position, $x_{\text {track}}$, at which the track crosses a given pad row (where the $y$ coordinate of the pads in the row are $y_{\text {row }}$ ) is given by equation 4.3 ,

$$
x_{\text {track }}=x_{0}+y_{\text {row }} \tan \left(\phi_{\text {local }}\right)
$$

In equation 4.3 the angle $\phi_{\text {local }}$ is the azimuthal angle of the track when it crosses a pad with midpoint at $y_{\text {row }}$.

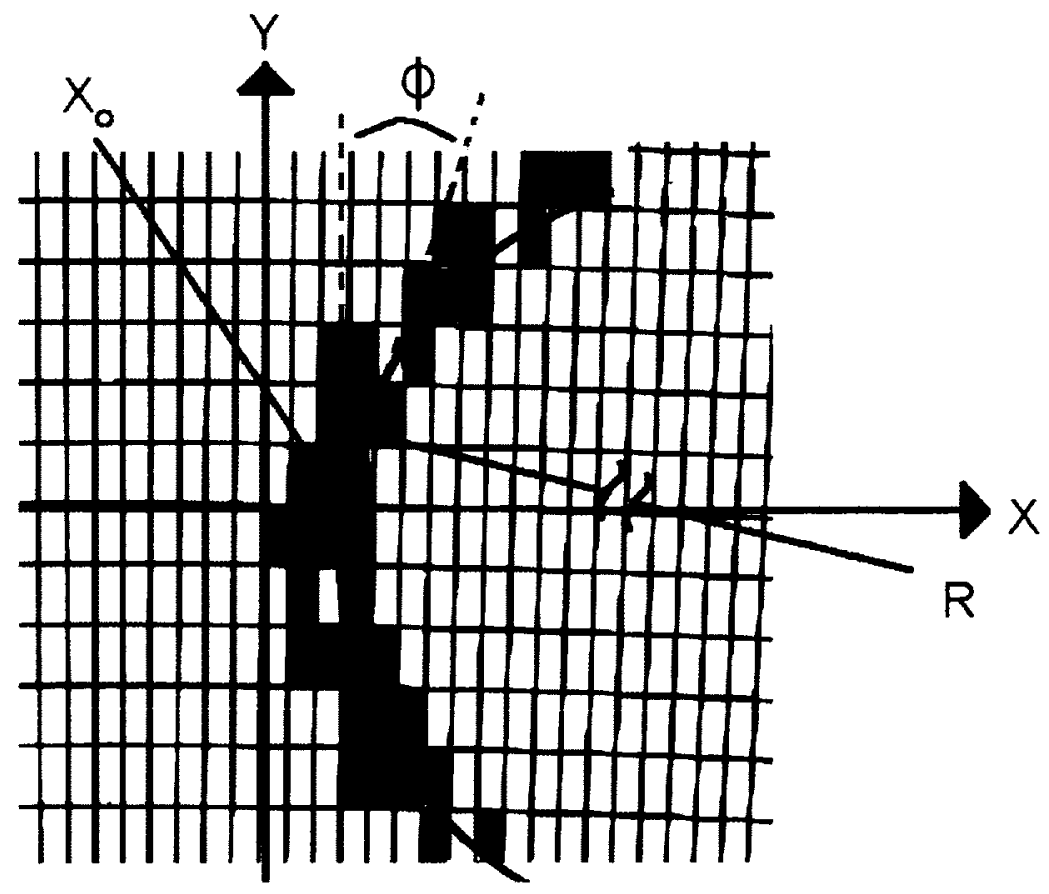

Figure 4.1: Example of track fitting to an event. Radius of curvature exaggerated to demonstrate track parameters. 
A chi-square minimization is used to determine the track parameters. The chisquare minimized to fit the track is given in equation 4.4 ,

$$
\chi_{\text {track }}^{2}=\sum_{i}^{n} \chi_{\text {row }}^{2}=\sum_{i}^{n} \sum_{j}^{m}\left[\frac{A_{j}^{i}-A_{\text {peak }}^{i} P R F\left(x_{j}^{i}-x_{\text {track }}\right)}{\sigma_{A_{j}^{i}}}\right]^{2}
$$

Here the first sum is over all the pad rows used and the second is over the pads in each row which recorded an amplitude above a threshold value (in figure 4.1 those would be the coloured pads). The variables $A_{j}^{i}$ and $x_{j}^{i}$ are the amplitude and position of the $j^{\text {th }}$ pad in the $i^{\text {th }}$ row. The scaling factor, $A_{\text {peak }}^{i}$, is calculated for each row individually by minimizing $\chi_{\text {row }}^{2}$ with respect to $A_{\text {peak }}^{i}$. The value of $A_{\text {peak }}^{i}$ which minimizes $\chi_{\text {row }}^{2}$ is found by taking the derivative of $\chi_{\text {row }}^{2}$ with respect to $A_{\text {peak }}^{i}$ and setting the result equal to zero. Then solving for $A_{\text {peak }}^{i}$ finds the value of $A_{\text {peak }}^{i}$ which minimizes $\chi_{\text {row }}^{2}$. The result of this process is given in equation 4.5 ,

$$
A_{\text {peak }}^{i}=\frac{\sum_{j}^{m} \frac{A_{j}^{i} P R F\left(x_{j}^{i}\right)}{\sigma_{A_{j}^{i}}^{2}}}{\sum_{j}^{m} \frac{P R F^{2}\left(x_{j}^{i}\right)}{\sigma_{A_{j}^{i}}^{2}}},
$$

where $x_{j}^{i}$ and $A_{j}^{i}$ are the same as in equation 4.4. The error, $\sigma_{A_{j}^{i}}$, in equations 4.4 and 4.5 was set to the square root of the pad amplitude.

The initial guess of the track used in the chi-square minimization is called the seed track. The pads in each row with the largest amplitudes, called the primary pads, are used to determine the seed track. A linear least squares fit is applied to the positions of the primary pads and the resulting fit parameters define the seed track. The inverse radius of curvature of the seed track is always set to 0 . 


\subsection{Analysis Pipeline}

This section will describe the analysis pipeline starting from the raw pad signals and ending with the calculation of the detector single hit $\mathrm{r} \phi$ resolution. The analysis is made up of four separate steps. These steps can be seen schematically in figure 4.2 .

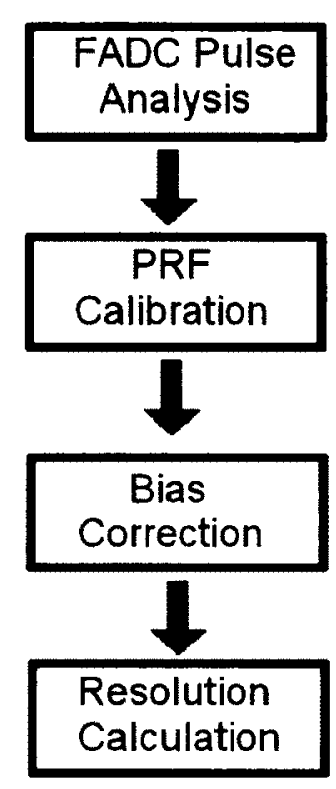

Figure 4.2: Schematic of the analysis pipeline.

The analysis pipeline is applied to each run individually. The first of these steps computes a pad amplitude and time measurement from the shaped pulses of each pad. The time measurement is not used in the calculation of the transverse resolution but will be included in this description for completeness. These values are then stored in what are called dense data (DD) files. The next step uses the values in the DD files to calibrate the PRF for the given run. Once the PRF has been properly calibrated the next step is to calculate a bias correction for each pad row in the detector. Finally, the DD file, PRF, and bias corrections are used to calculate the resolution of the detector. These four steps in the the analysis pipeline will be described in more detail in the following subsections. 


\subsubsection{Calculation of Pad Amplitudes}

The amplitude and time are calculated using some defining feature of the shaped pad pulses. There are three methods which have been used to determine the amplitude and time measurement. This section will give a description of each method.

The pulse analysis method which had been used in previous studies is known as the Single Point Maximum (SPM). This defines the amplitude as the largest FADC value in the pulse and uses the value of the time bin of the largest pulse as the time measurement. While this can produce a useful value for amplitude, the time measurement is quantized to the widths of the FADC bins (either 40 ns or 20 ns depending on the sampling frequency used). As well, both the time and amplitude measurements are significantly affected by noise. This effect is more pronounced in pads with lower pulse heights as the amount of noise is independent of pulse height. An example of this SPM method is shown in figure 4.3.a.

An improvement to the SPM method is the Quadratic Fit Method (QFM). This method finds the largest FADC value and the four time bins surrounding it. These five points are then used to fit a quadratic function (see figure 4.3.b). The maximum point of the fitted quadratic is then used to define the time and amplitude measurements. This method improves both the amplitude and the time measurements compared to the SPM. The time value is no longer quantized to the time bin widths of the FADC. As well, both the time and amplitudes are less affected by noise since the fit averages the values over more than one time bin. This method of pulse analysis will be used to produce baseline measurements of the $\mathrm{r} \phi$ resolution. These baseline measurements will then be compared to the results obtained using the new pulse analysis technique described next.

The new method, which is the focus of this work, is used presently only for determining the amplitude and would require another method for determining the time 

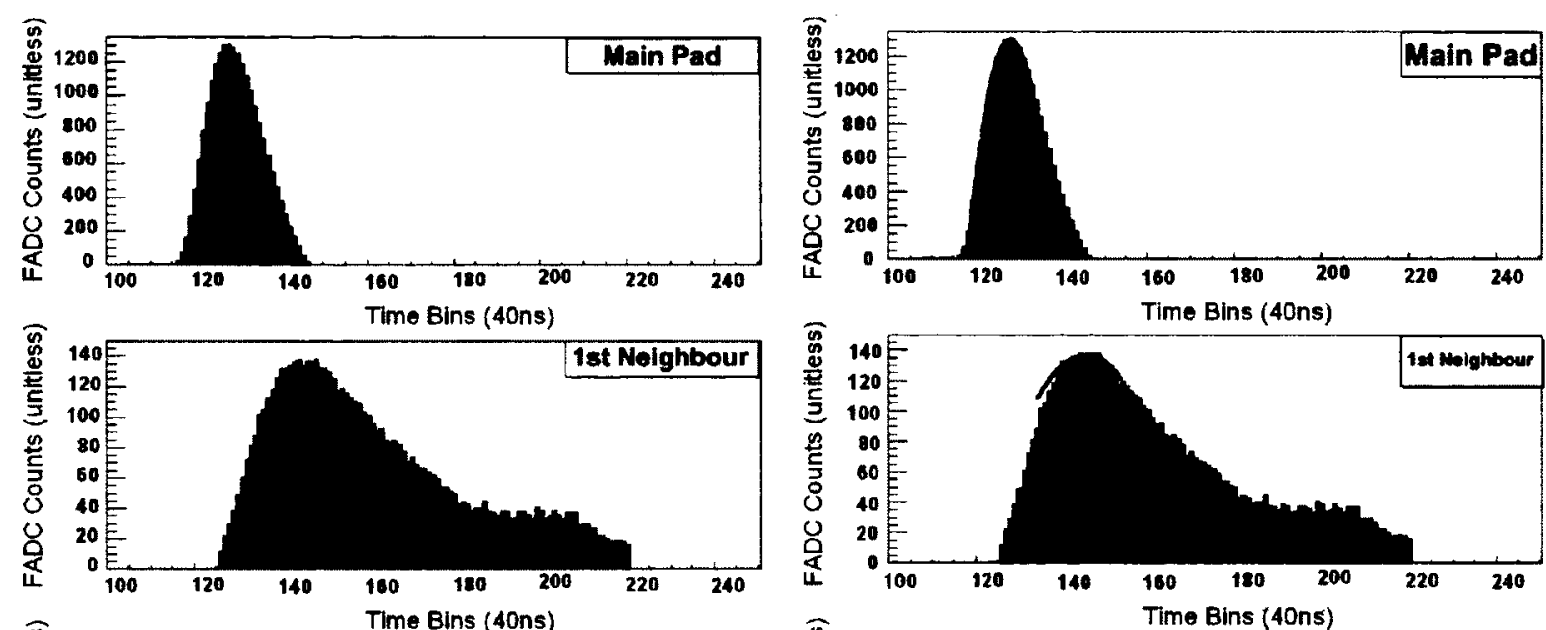

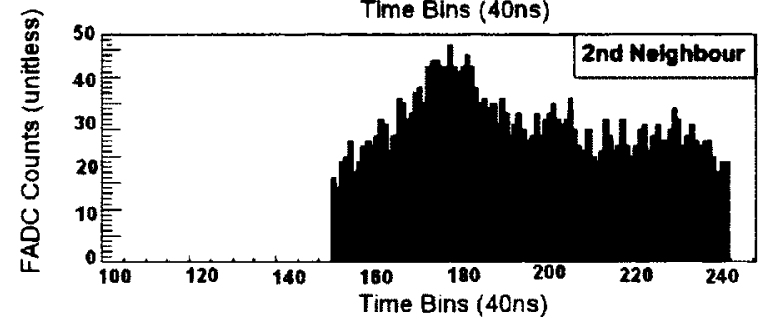

(a)

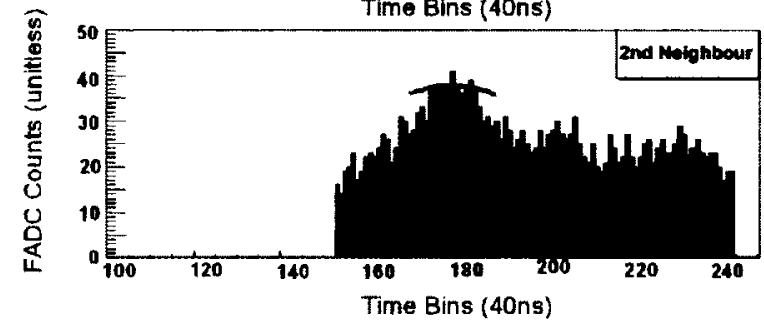

(b)

Figure 4.3: Examples of the pulse height analysis techniques. a) Single Point Maximum (SPM), b) Quadratic Fit Method (QFM).

measurement. The amplitude is calculated by integrating the FADC pulse over a fixed window. The integration is defined by two numbers: the starting time bin, $T_{\text {start }}$, and the integration width, $\omega$. Presently the same width is used for an entire scan and is calibrated to give the best resolution. The starting time bin changes between rows depending on the FADC pulse of the pad in each row with the largest signal and is found using the following method. The RMS of the noise, $\sigma_{r m s}$, is calculated from the first 20 bins of the FADC pulse. The RMS of the noise is found by first calculating the average FADC value, $\bar{A}$, in the first 20 bins. Next $\bar{A}$ is used to find $\sigma_{r m s}$ using equation 4.6 .

$$
\sigma_{r m s}=\sqrt{\frac{\sum_{i=1}^{N}\left(A_{i}-\bar{A}\right)^{2}}{N-1}},
$$

where $A_{i}$ the FADC value in the $i^{t h}$ time bin, and $\mathrm{N}$ is the number of time bins used 
(20 in this case). To avoid signal being located in the bins used to calculate the RMS of the noise extra time bins were record before the actual signal arrived (see figure 4.4). The first time bin, $T_{\text {signal }}$, with a signal greater then $4 \sigma_{r m s}$ is then located. This time bin is located in the actual pulse. To ensure the beginning of the pulse is included in the window, $T_{\text {start }}$ is located 3 time bins before $T_{\text {signal }}$, (see figure 4.5 ). The pad amplitude is then calculated using equation 4.7,

$$
A=\sum_{i=t_{\text {start }}}^{t_{\text {start }}+\omega} A_{i}
$$

where $A_{i}$ is the value in the $i^{\text {th }}$ FADC time bin and $\mathrm{A}$ is the resulting integrated pad amplitude.

The rationale for the Reintegration Method is as follows. Short shaping times are not long enough to fully integrate the preamplifier pulse. As a result the peak height of a short shaping time pulse is not an accurate measure of the charge seen by the pad. The information contained in the entire shape of the pulse, however, can be used to get a better estimate of the total charge seen by the pad. The Reintegration Method uses the information contained in the shape of the pad pulse to calculate the pad amplitude. The pad amplitude calculated using the Reintegration Method is then a better estimate of the total charge seen by the pad.

\subsubsection{PRF Calibration}

The PRF could be measured directly if an external measure of the track position was available. A similar measurement was conducted using a fixed collimated x-ray source [16]. Since no precise external measurement of the track is available for the LP-TPC, the PRF must be calibrated using another method. The technique uses an iterative approach which requires an initial guess of the PRF parameters. It relies on internal consistency of the data to ensure an appropriate PRF is found. 


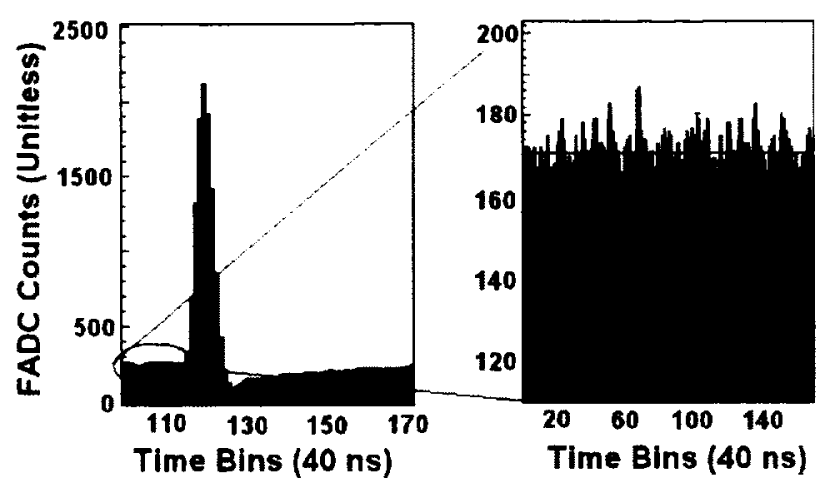

Figure 4.4: Example signal pedestal calculation used in Reintegration Method.
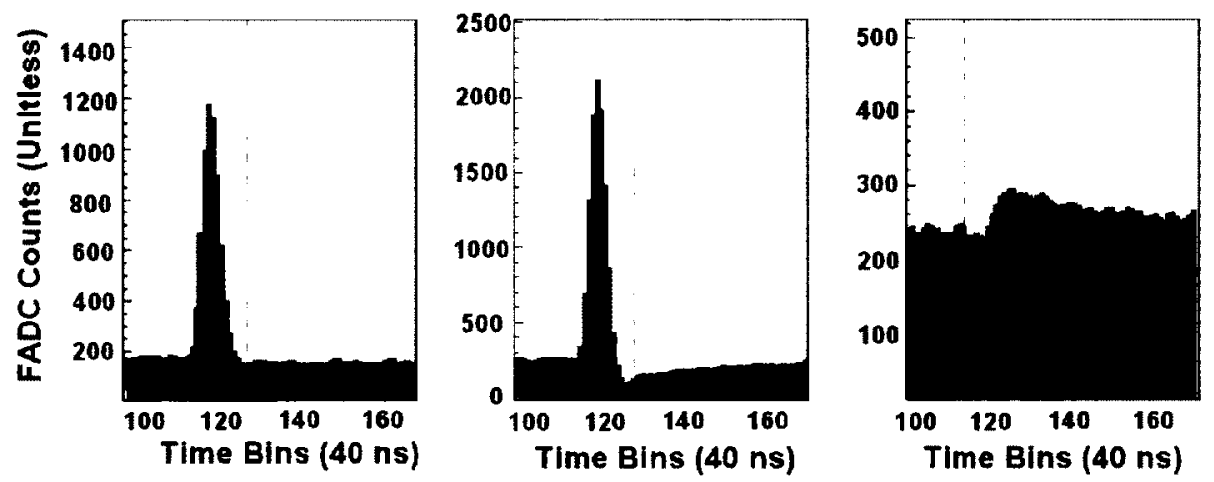

Figure 4.5: Example of Reintegration method applied to pad pulses (integration width 15 time bins). Note the pad with the largest amplitude contains an undershoot which is contained in the integration region. This does not negatively affect the amplitude calculation since the integral is used capture the information contained in the "shape" of the pulse.

The iterative process is used to calibrate the PRF for each run in a scan. The process uses a set of test parameters $\left(r_{i n}, w_{\text {in }}\right)$ to find a new set of parameters $\left(r_{\text {out }}, w_{\text {out }}\right)$ which better describes the data. The flow chart shown in figure 4.6 describes the steps used in the iterative calibration process. 


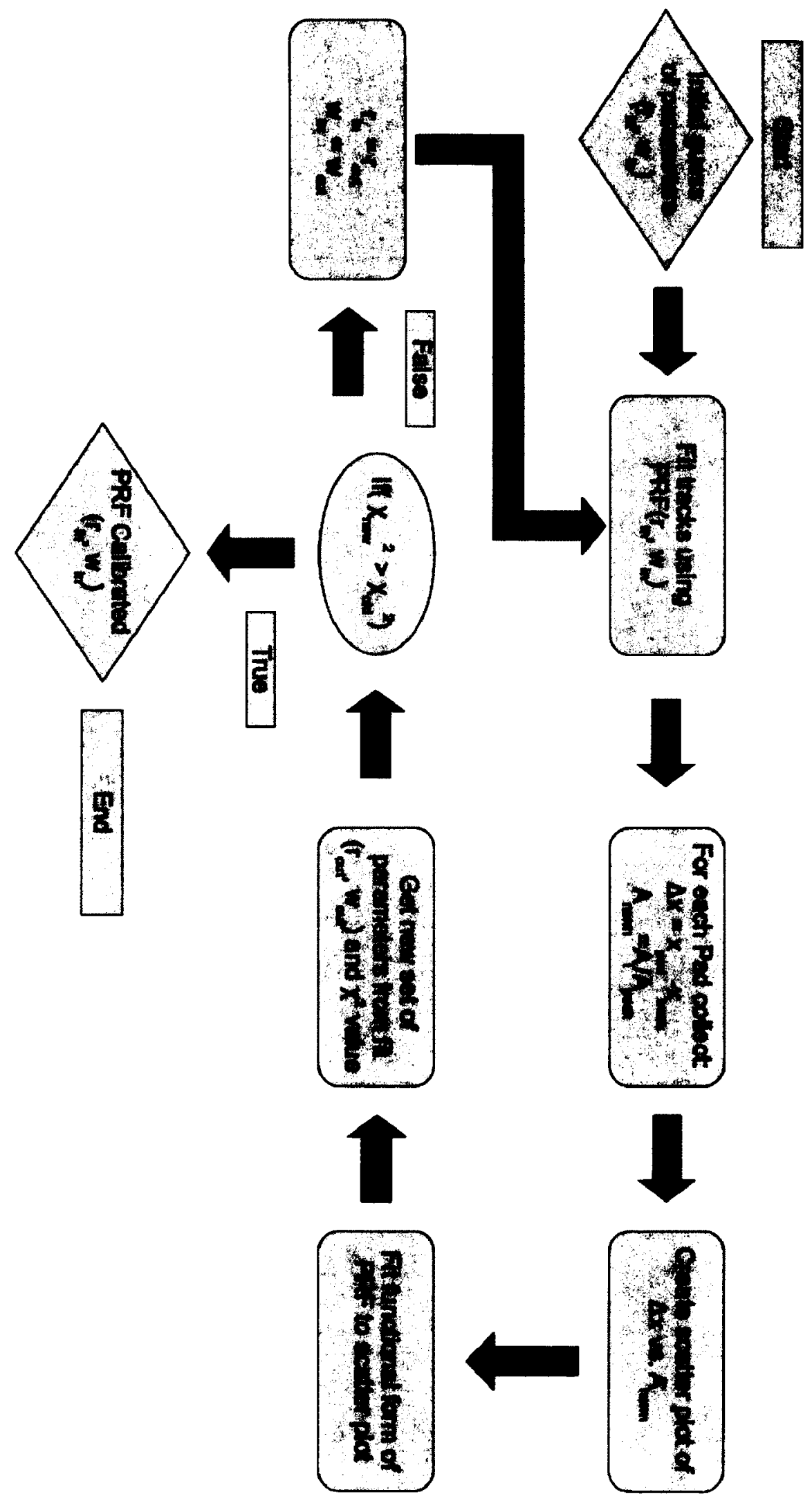

Figure 4.6: This flow chart shows the steps used in the iterative PRF calibration process. 
To start the iterative process, initial guesses of the PRF parameters are required. For most runs initial guesses of $r=0.5$ and $w=3.5$ were used. This started the PRF as half Gaussian, half Lorentzian, and with a width about the same as a single pad. The initial set of PRF parameters was then used to perform all the track fitting in the run. Only events which passed the event selection rules described in appendix A were used. After tracks have been fit to all the successful events, the values shown in equations 4.8 and 4.9 were recorded for each pad with an amplitude greater than some threshold value, determined by noise.

$$
\begin{gathered}
\Delta x=x_{\text {pad }}^{i}-x_{\text {track }} \\
A_{\text {norm }}=A_{i} / A_{\text {peak }}
\end{gathered}
$$

In equation $4.8 \Delta x$ is the distance between the $i^{\text {th }}$ pad (located at $x_{p a d}^{i}$ ) and the track (located at $x_{\text {track }}$ ). In equation $4.9 \mathrm{~A}_{\text {norm }}$ is the normalized amplitude of the $i^{\text {th }}$ pad, $\mathrm{A}_{i}$ is the amplitude of the $i^{\text {th }} \mathrm{pad}$, and $\mathrm{A}_{\text {peak }}$ is the peak amplitude described in section 4.2 .

The values $\Delta x$ and $A_{n o r m}$ are then used to create a scatter plot of $\Delta x$ versus $\mathrm{A}_{\text {norm }}$. An example of such a scatter plot is shown in figure 4.7. This scatter plot is the combined measurement of the PRF from all events in the run.

The scatter plot is then divided into 86 bins of equal width and the average of each bin is calculated. These 86 points are then used to fit the functional form (equation 4.1) of the PRF, figure 4.8. From the fit two new parameters $\left(r_{\text {out }}, w_{\text {out }}\right)$ and the chisquare value $\chi_{n e w}^{2}$ of the fit are retrieved. To determine whether the new parameters better describe the data $\chi_{\text {new }}^{2}$ is compared to the total chi-square value of the previous iteration, $\chi_{\text {old }}^{2}$.

If $\chi_{\text {new }}^{2}$ is smaller than $\chi_{\text {old }}^{2}$, then the PRF defined by $\left(r_{\text {out }}, w_{\text {out }}\right)$ better describes the data and these parameters are used as the initial guess of the next iteration. 


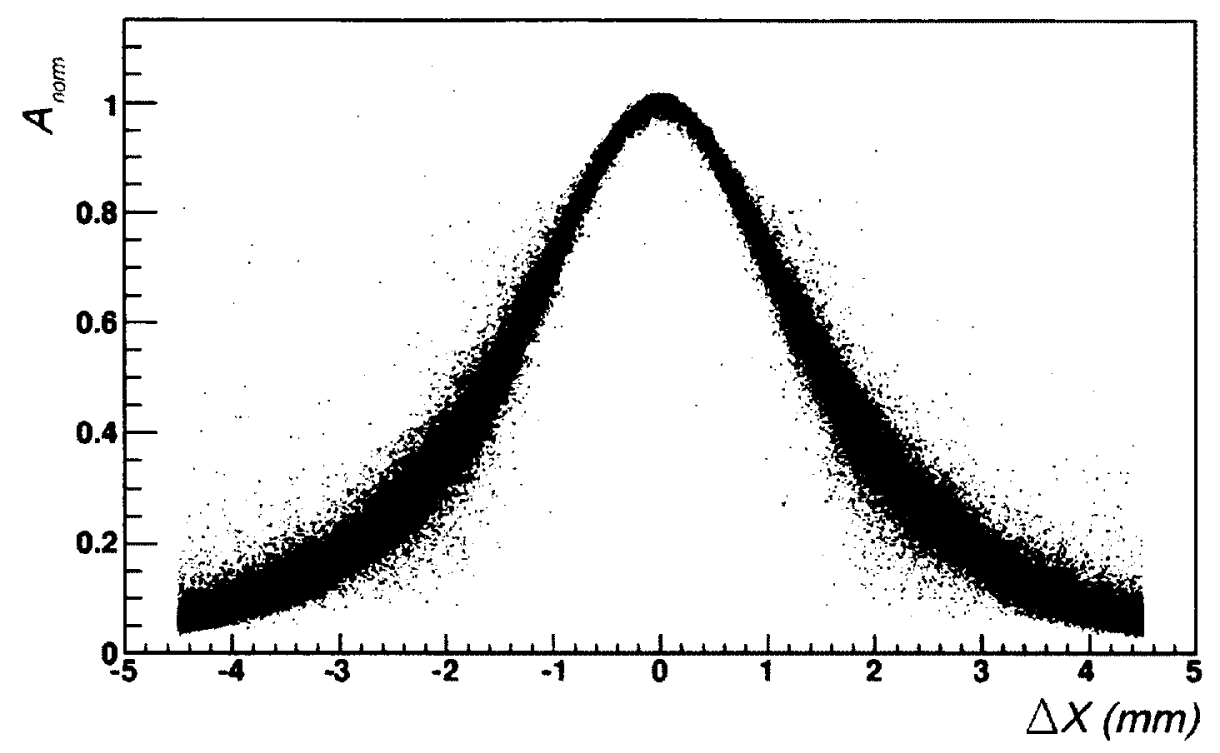

Figure 4.7: Example scatter plot of $\Delta X$ vs. $A_{\text {norm }}$. From the the PRF calibration of run 1230 .

Otherwise, $\chi_{\text {new }}^{2}$ is greater than $\chi_{\text {old }}^{2}$, the iterative process is ended and the parameters $\left(r_{i n}, w_{i n}\right)$ are the properly calibrated PRF parameters. In the case of the first iteration where no $\chi_{\text {old }}^{2}$ value is available the comparison is skipped and a second iteration is automatically conducted.

To test whether the iterative approach produces a valid PRF, the behaviour of the PRF parameters as a function of drift distance is checked. This check is donc after the PRF has been determined for a full scan of the TPC. One would expect the parameters to display a smoothly varying dependence on drift distance. In particular, the PRF is expected to become wider with increasing drift distance. This widening is due to diffusion, and charge dispersion.

For the present functional form of the PRF the $r$ parameter is expected to decrease and the w parameter is expected to increase as a function of drift distance. Both of these behaviours are caused by the increase in diffusion resulting from an increase in drift distance. An electron cluster which drifts further will become wider due to 


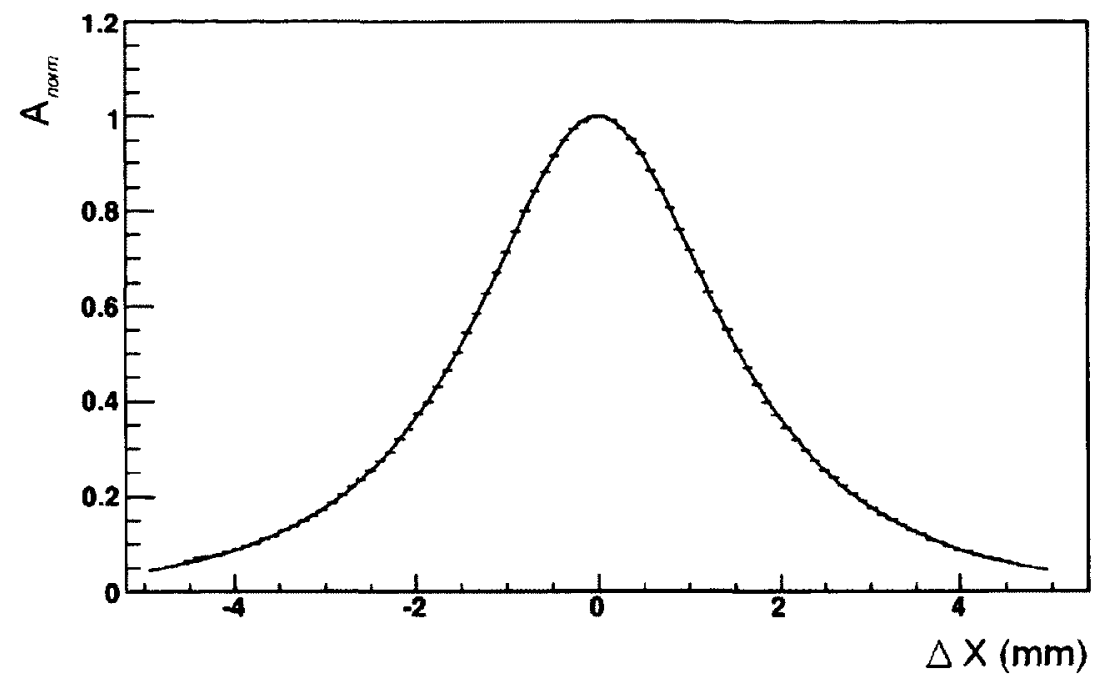

Figure 4.8: Example fit of equation 4.1 to the binned scatter plot of $\Delta x$ vs. $A_{n o r m}$. From the PRF calibration of run 1230.

diffusion. As the cluster becomes more diffuse the amount of charge dispersing from any one point decreases and the charge dispersion signals becomes less prominent. This will result in a wider and more Gaussian PRF. Diffusion causes the electron cluster to take on a Gaussian profile and this results in an increase in the weight of the Gaussian in the PRF. To account for this, the r parameter becomes smaller which increases the weight on the Gaussian portion of the PRF. The increase of w is natural because it is the width parameter of both the Gaussian and Lorentzian.

\subsubsection{Bias Corrections}

Due to imperfections in the construction of the resistive anode assembly leading to local variations in the $\mathrm{RC}$ parameters, each row has a position dependent systematic error associated with its track position determination. A bias correction is calculated to account for this systematic error before the final resolution of the detector is measured. Bias corrections are calculated separately for each row. They are calculated as a function of position along a row and are made up of averaged row residuals. This 
section will explain how equation 4.2 is used to calculate row residuals. It will then explain how the bias correction is found from the row residuals and how it is applied.

Before a more rigorous description of row residuals is given, and how they are used to calculate bias corrections and resolution, a more qualitative description will be discussed.

Since no external measure of a track is available, a comparison between the true track and measured track is not possible. This would be the typical way of accessing the resolution of the detector. However, by relying on the internal consistency of the track positions measured by each pad row in the TPC, a similar comparison can be made.

To determine the resolution of the detector overall first the resolution of each pad row is measured. These row resolutions can then be combined later to determine the resolution of the detector. To measure the resolution of a single row, called the test row, a more accurate determination of the track must be made then can be achieved by the test row alone. This more accurate measurement, the inclusive or exclusive track fit mentioned previously, is achieved by using all the pad rows (inclusive track fit) or all the pad rows except the test row (exclusive track fit) in the detector to fit a track. The test row is then used individually to determine the position of the track above the test row. This measurement of the track position by the test row is called the single row track fit. The position predicted by the inclusive or exclusive track fit can then be compared to the position predicted by the single row track fit. The difference between the predicted track positions is called the row residual (of the test row). In the case where the inclusive track fit is used it is called the inclusive row residual, and in the case where the exclusive track fit is used it is called the exclusive row residual.

The reason for the two types of track fits (inclusive and exclusive) is that the residual calculated by each individually are incorrect. The inclusive row residual is 
too small and the exclusive row residual is too large. However when combined using equation 4.2 they produce a more accurate estimate of the true row residual.

By collecting the row residuals calculated from equation 4.2 a measurement of the error in the determination of track position by a row can be made. How this can then be used to correct for position dependent bias in a row is discussed in section 4.3.3. How the row residuals are used to calculate the detector resolution is then described in section 4.4 .

\section{Row Residuals}

As mentioned in the analysis overview (section 4.1) the row residual is estimated using equation 4.2. This equation estimates the row residual of a test row using the so-called inclusive, $\sigma_{i n}$, and exclusive, $\sigma_{e x}$, track fit row residuals. An inclusive row residual is calculated from a track fit which includes the test row. An exclusive row residual is calculated from a track fit which does not include the test row. As mentioned previously both inclusive and exclusive residuals are required because individually their estimates are incorrect.

The inclusive and exclusive row residuals are calculated using equation 4.10 and 4.11 , respectively.

$$
\begin{gathered}
\sigma_{i n}=x_{t r a c k}^{i n}-x_{t r a c k}^{\prime}, \\
\sigma_{e x}=x_{t r a c k}^{e x}-x_{t r a c k}^{\prime},
\end{gathered}
$$

where $x_{\text {track }}^{\text {in }}$ and $x_{\text {track }}^{e x}$ are the track positions on the test row for the inclusive and exclusive track fits respectively. The position $x_{\text {track }}^{\prime}$ is the single row track fit. The single row track fit is calculated by minimizing the chi-square in equation 4.12 with 
respect to $x_{t r a c k}^{\prime}$.

$$
\chi_{\text {row }}^{2}=\sum_{i}^{n}\left[\frac{A_{i}-A_{\text {peak }} P R F\left(x_{i}-x_{\text {track }}^{\prime}\right)}{\sigma_{A_{i}}}\right]^{2}
$$

Where $x_{\text {track }}^{\prime}$ is the position of the track on the test row as determined by the test row itself.

\section{Calculation of Bias Correction}

The bias correction is calculated after all the row residuals have been found. The residual for each row and the inclusive track fit parameters are recorded. Using the inclusive track parameters the track position on each row, $x_{t r a c k}^{i n}$, is calculated. This value is then plotted against the row residual for that track in separate plots for each row. After all the tracks have been analyzed a scatter plot is produced for each row. Figure 4.9 shows an example of such a scatter plot for the $13^{\text {th }}$ pad row of the readout plane.

The scatter plot is then binned into forty bins and the average residual in each bin is calculated. These forty points sample the bias correction function at equally spaced steps (step size is $0.5 \mathrm{~mm}$ ). Figure 4.10 below shows an example of the resulting histogram after binning and averaging the scatter plot shown in figure 4.9. A spline interpolation is used to evaluate the bias correction function between points.

The bias correction is used during the track fitting and row residual calculation processes. The correction is applied whenever the PRF must be evaluated on a row. For example, when performing the single row track fit or the inclusive/exclusive fit the minimized $\chi_{\text {row }}^{2}$ becomes,

$$
\chi_{\text {row }}^{2}=\sum_{i}^{n}\left[\frac{A_{i}-A_{\text {peak }} P R F\left(x_{i}-x_{\text {track }}^{\prime}+\operatorname{Bias}\left(x_{\text {track }}\right)\right)}{\sigma_{A_{i}}}\right]^{2},
$$




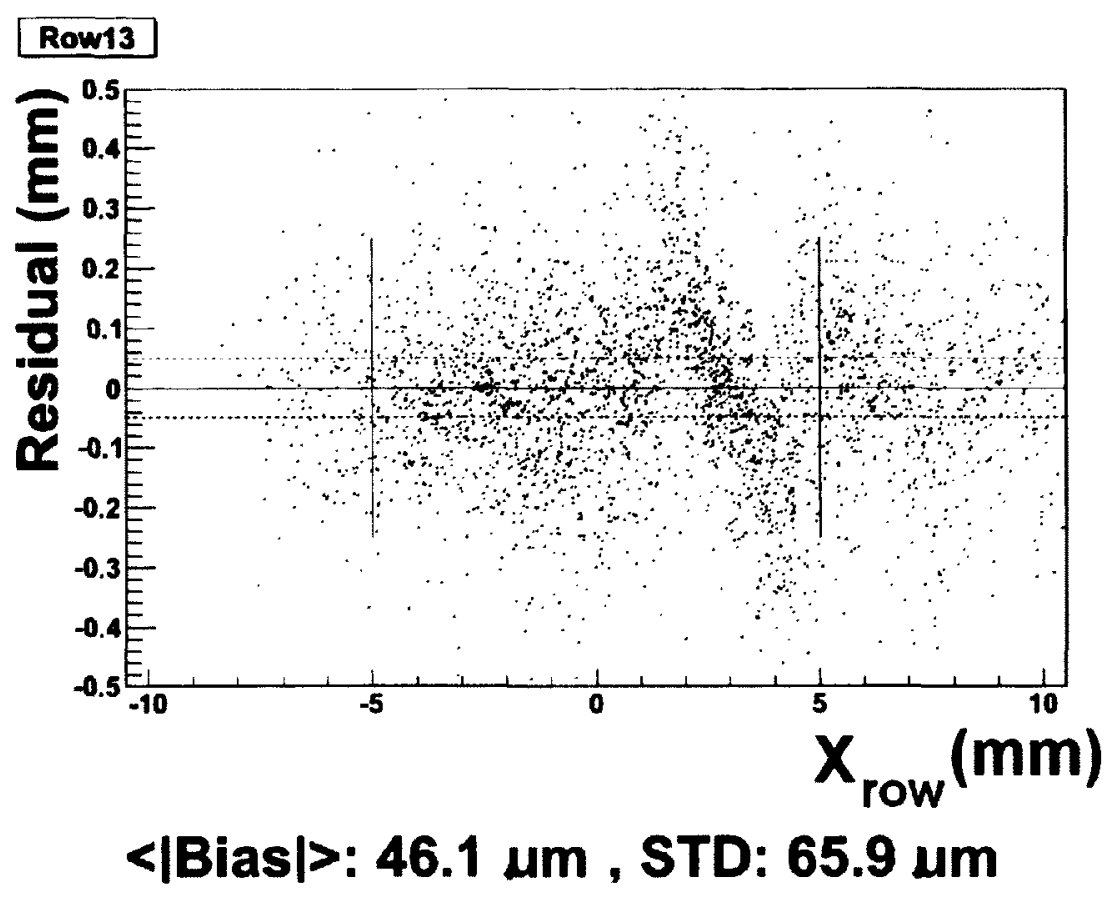

Figure 4.9: Example of a scatter plot of row residual verses $x_{\text {track }}^{\text {in }}$ for the 13th row of the detector.

where $\operatorname{Bias}\left(x_{t r a c k}\right)$ is the bias correction function evaluated at the point where the track fit crosses the row.

After applying the bias correction to the track fitting the resolution can be calculated. Figures 4.11 and 4.12 show the row residual of row 13 after the application of the corrections. Note the reduction in row residuals across all pads in the row.

Two other consistency checks are conducted after the application of the bias corrections. After the row residuals for each row are plotted as in figures 4.10 and 4.12 two values are calculated. The average absolute value of the residuals and the standard deviation of the absolute value of the residuals are calculated (they can be seen under the $\mathrm{x}$-axis of figures 4.10 and 4.12). These values are then compared for the row residual distributions before and after the application of the bias correction. If the average and standard deviation both decrease then the bias correction has been calculated and applied correctly. 


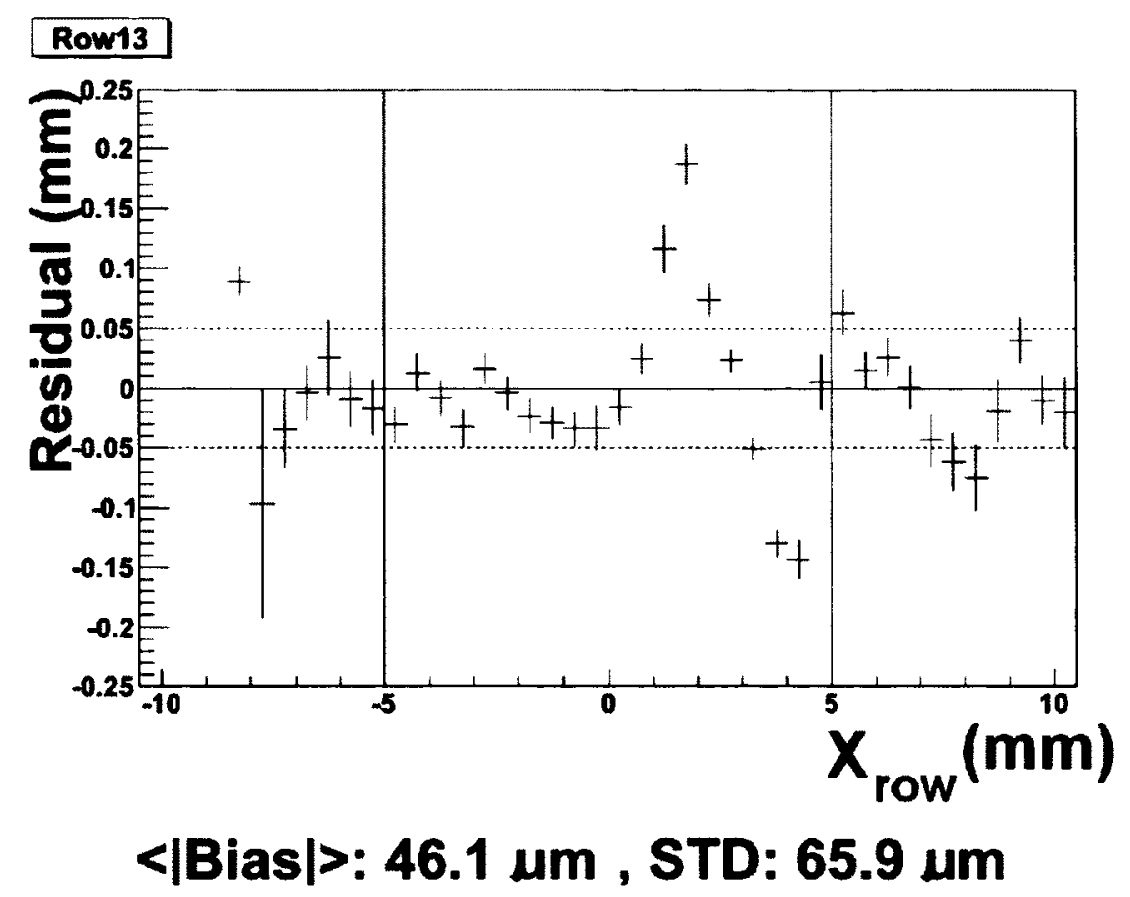

Figure 4.10: Example of a binned and average row residual scatter plot for the 13th row of the detector.

\subsection{Resolution Measurement}

The single hit $\mathrm{r} \phi$ resolution is measured from the global (global meaning from all the rows in all the events) row residual distribution after the application of the bias corrections. The single hit $\mathrm{r} \phi$ resolution of the detector is defined as the width of the global row residual distribution. The width is found by fitting the residual distribution to a normal distribution. Example global residual distributions and corresponding normal distribution fits for five runs $(1226,1227,1228,1229$, and 1230) are shown in figure 4.13 .

The global residual distribution does not include the row residuals calculated from the $12^{\text {th }}$ (bottom) and $22^{\text {nd }}$ (top) rows. This is because the residuals in these rows are calculated from an extrapolation of the fitted track as opposed to an interpolation. This results in a significantly wider row residual distribution for rows 12 and 22 than 


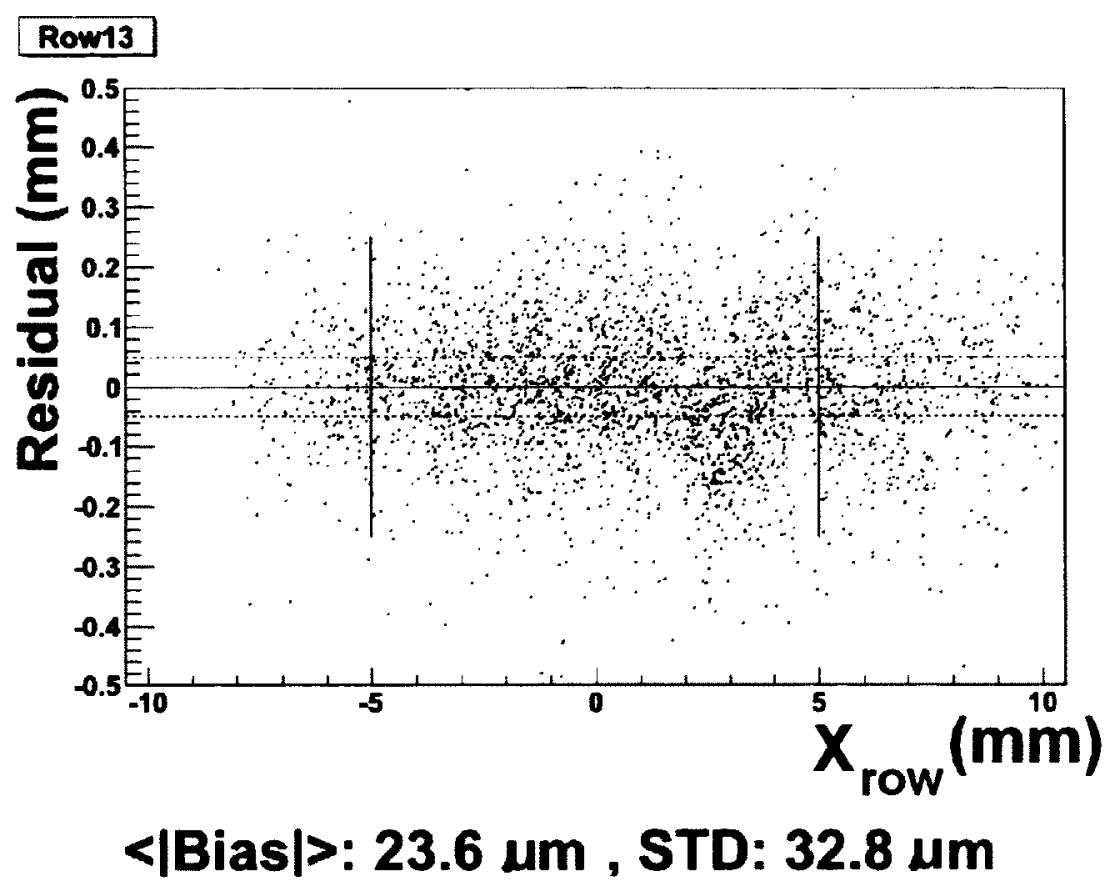

Figure 4.11: Example of a scatter plot for the 13th row of the detector after the application of the bias corrections.

for the middle pad rows. When performing the exclusive track fit on a test row in the middle of the readout module, the rows above and below the test row constrained the fitted track. However, when the exclusive track fit is performed on the top and bottom rows the fitted track is not constrained. The resulting track does not take into account the trend of the top or bottom row.

\subsection{Dependence of Resolution on Drift Distance}

After all the runs in a scan have been analyzed the relationship between single hit $\mathrm{r} \phi$ resolution and drift distance can be plotted (see figure 5.9). If the resolution dependence on drift distance is consistent with the effects of diffusion and electron statistics, as has been observed in previous studies [20] [25] [24], then the measured 


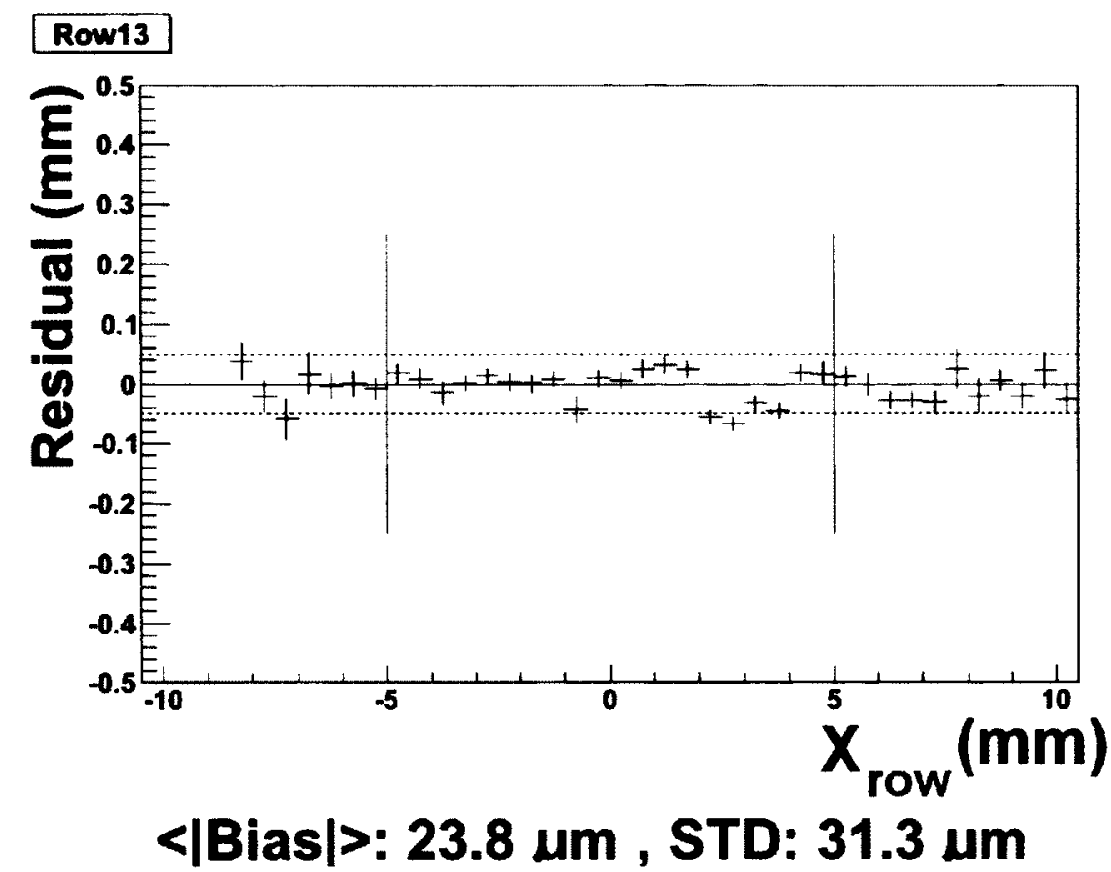

Figure 4.12: Example of a binned and average row residual scatter plot for the 13th row of the detector after the application of the bias corrections.

resolution as a function of drift distance should follow equation 4.14.

$$
\sigma(z)=\sqrt{\sigma_{0}^{2}+\frac{D_{t r}^{2} z}{N_{e f f}}}
$$

Equation 4.14 shows the single point transverse resolution has 2 components. The first component, $\sigma_{0}$, is the intrinsic component of resolution resulting from noise in the detector. The other component is the result of diffusion which depends on the width of the electron cluster $D_{t r}^{2} z$ and the effective number of electrons observed $n_{\text {eff }}$. When combined as in equation 4.14 these two terms are the variance of the estimated mean position of an electron cluster from a sample size of $n_{\text {eff }}$.

Equation 4.14 function is fitted to the measurements of transverse resolution. Before equation 4.14 can be used to fit the experimentally measured resolution, a value for $D_{t r}$ must be found. As mentioned previously (section 2.2.2) this value can 


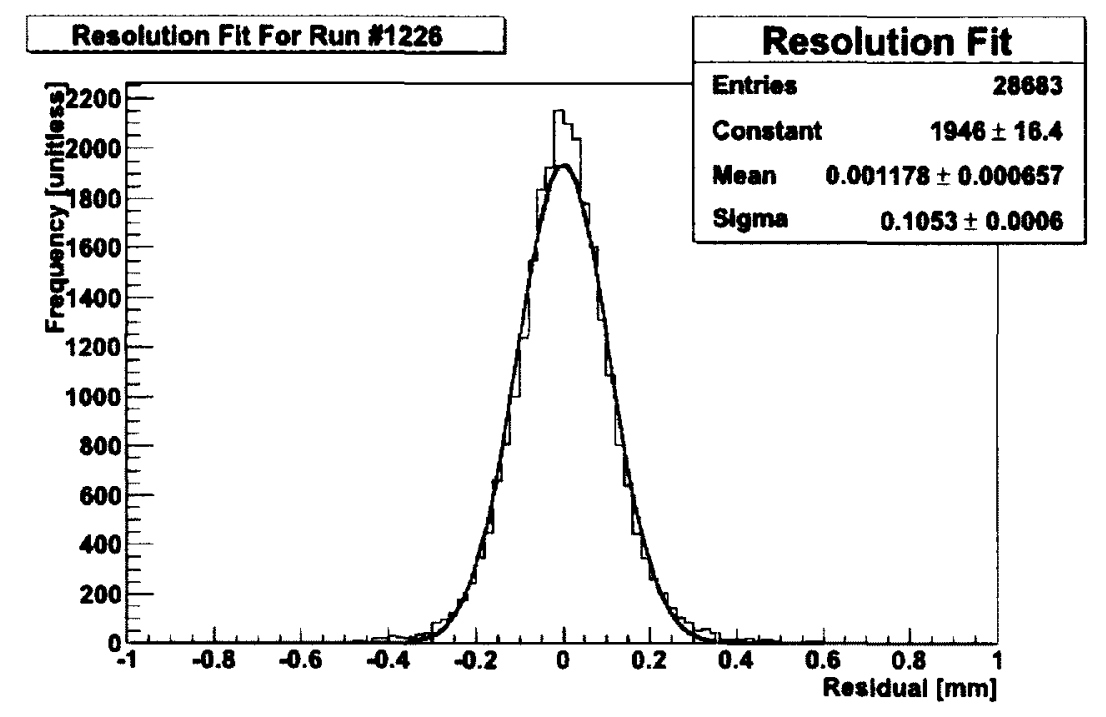

(a)

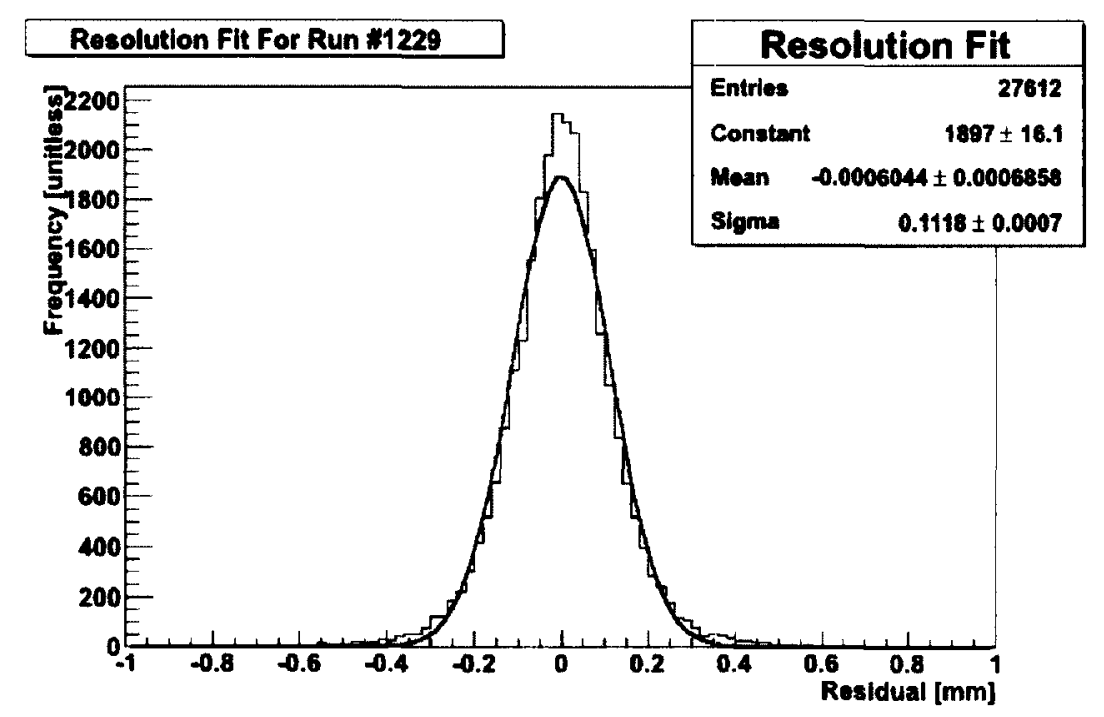

(c)

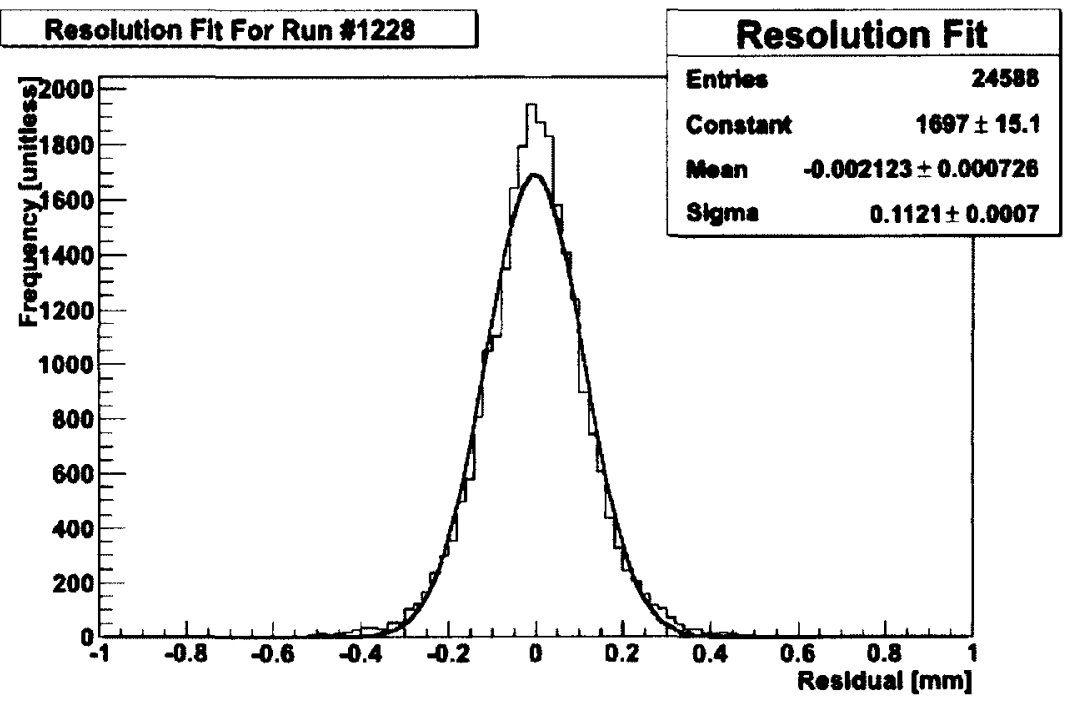

(b)

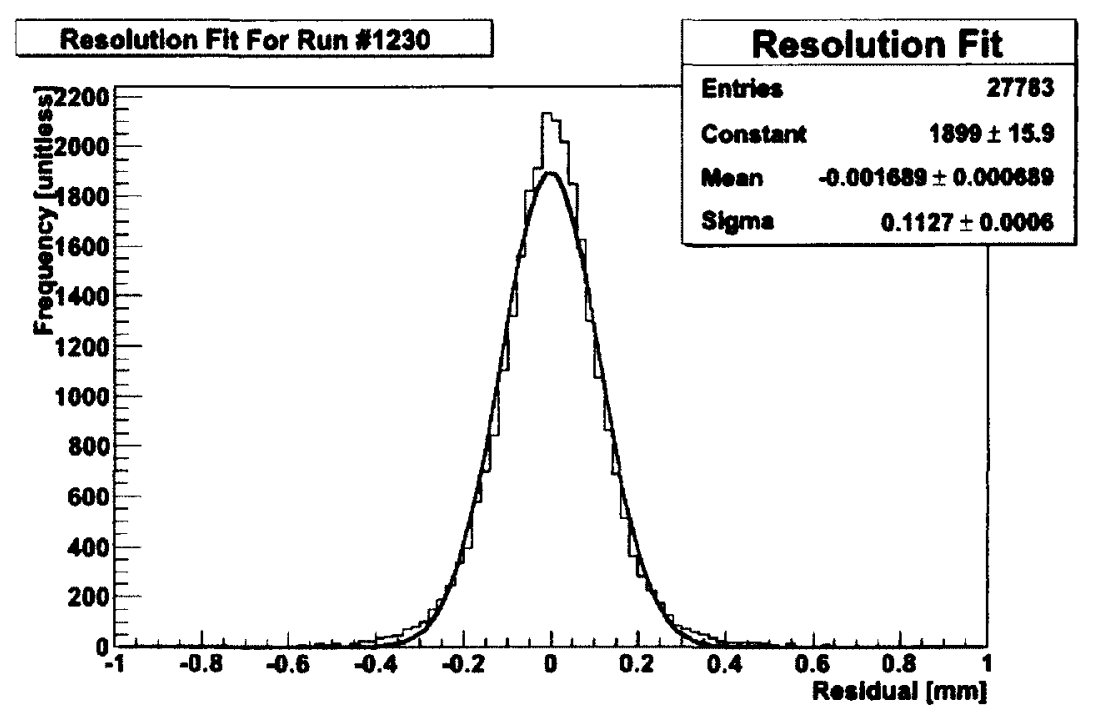

(d)

Figure 4.13: Examples of the global residual distributions and corresponding normal distribution fits for for runs 1226 
be calculated using the Monte Carlo program Magboltz.

The resulting fit parameters $\left(\sigma_{0}\right.$ and $\left.N_{\text {eff }}\right)$, from the fit to scan 5 measurements, were also used to extrapolate the $\mathrm{r} \phi$ resolution found using the Reintegration Method to $2 \mathrm{~m}$ of drift. To complete the extrapolation the coefficient of transverse diffusion must be recalculated. This recalculation is required to due the dependence of $\mathrm{D}_{T r}$ on magnetic field strength. The measurements of the $\mathrm{r} \phi$ resolution made with the LP-TPC were conducted with a $1 \mathrm{~T}$ magnetic field, whereas the ILD-TPC will be in a $3.5 \mathrm{~T}$ magnetic field. 


\section{Chapter 5}

\section{Results}

The outcome of the intermediate analysis steps will be presented in the same order as the analysis was conducted. Firstly, the optimization of the integration width, needed for the Reintegration Method, will be presented. Secondly, the calibrated PRF parameters for all 5 scans will be shown along with the outcome of the PRF parameter validation test. Next the calculation and application of the bias corrections and the outcome of the bias consistency checks will be presented. Following this the results of the single hit $\mathrm{r} \phi$ resolution measurements for all 5 scans will be presented and compared. Finally, the extrapolation of the Reintegration Method resolution measurements to the ILD-TPC conditions will be presented.

\subsection{Optimization of Integration Width}

In order to use the Reintegration Method to calculate pad amplitudes, an integration width, $\omega$, must be chosen. This width will be the range over which the pad pulse will be integrated (as discussed in section 4.3.1). In order for the Reintegration Method to produce the best possible results, $\omega$ must be optimized. To determine the optimal integration width, $\omega$ was varied between 10 and 35 time bins and the resolution measured. Since the technique was applied to data collected at a FADC sampling 
rate of $25 \mathrm{MHz}$, this corresponds to integration widths between $400 \mathrm{~ns}$ and $1400 \mathrm{~ns}$. These optimization measurements were conducted using run 1226 (30 cm drift, 100 ns shaping time, none zero suppressed). The results of these calculations can be seen in Figure 5.1.

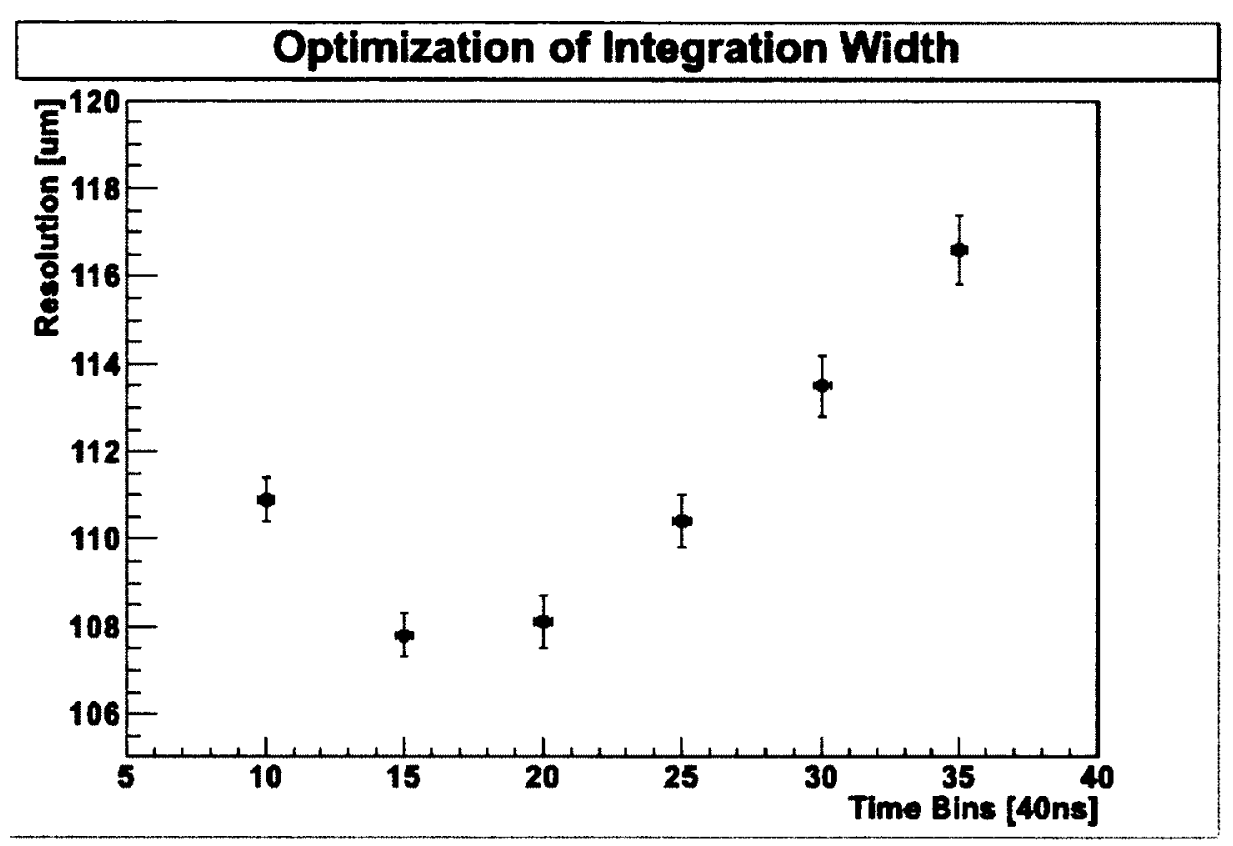

Figure 5.1: Determination of optimal integration width. Note errors on resolution are set equal to the error on the Gaussian width fit to the residual distribution.

From figure 5.1 it is seen that the optimal integration width is 15 bins. This value was applied to all the amplitude calculation in the runs of scan 5 .

\subsection{PRF Calibration}

Figures 5.2 and 5.3 show the calibrated PRF parameters for scans 1 through 4 (scan parameters listed in appendix B) as a function of drift distance. These scans were analyzed using the QFM pulse analysis technique. Figures 5.4 and 5.5 show the same plots for the PRF parameters obtained for scan 5, which was analyzed using the Reintegration Method. In both cases the calibrated PRF parameters satisfy the first 
validation check of the analysis, a smoothly varying dependence on drift distance (see section 4.3.2). The shape of the PRF is governed by diffusion which increases as a function of drift distance. For this reason the PRF parameters should also show a monotonic behaviour as a function of drift distance. The PRF parameters for both analysis techniques showed a monotonic behaviour with increased drift distance.

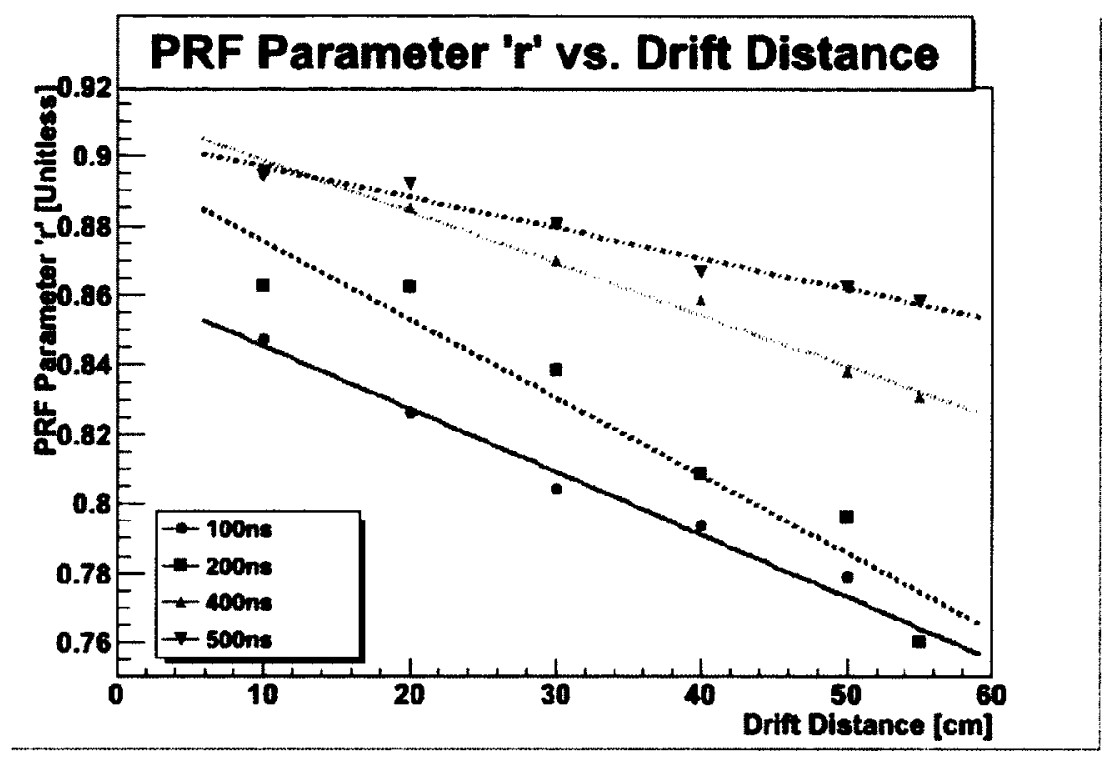

Figure 5.2: Calibration results for scans 1 through 4 of the PRF mixing parameter r. These scans were analyzed using the QFM method. An $r$ value of 1 produces a fully Lorentzian PRF and 0 a fully Gaussian PRF. The trend shows the PRF becoming more Gaussian-like as drift distance increases. Note errors (statistical component only) on PRF parameters to small to be seen on graph.

Figures 5.2 and 5.4 show that the mixing parameter $\mathrm{r}$ decreases with increased drift distance for both the QFM and Reintegration Method. This is consistent with diffusion, as discussed in section 4.3.2. The PRF of the Reintegration Method displays a more Gaussian shape than the QFM PRF, irrespective of shaping time. The Reintegration Method also shows a quicker decrease in the mixing parameter of its PRF than the QFM PRF. This is demonstrated by the quadratic dependence on drift distance seen in the mixing parameter of the Reintegration Method PRF as opposed to the linear dependence of the QFM PRF mixing parameter. 


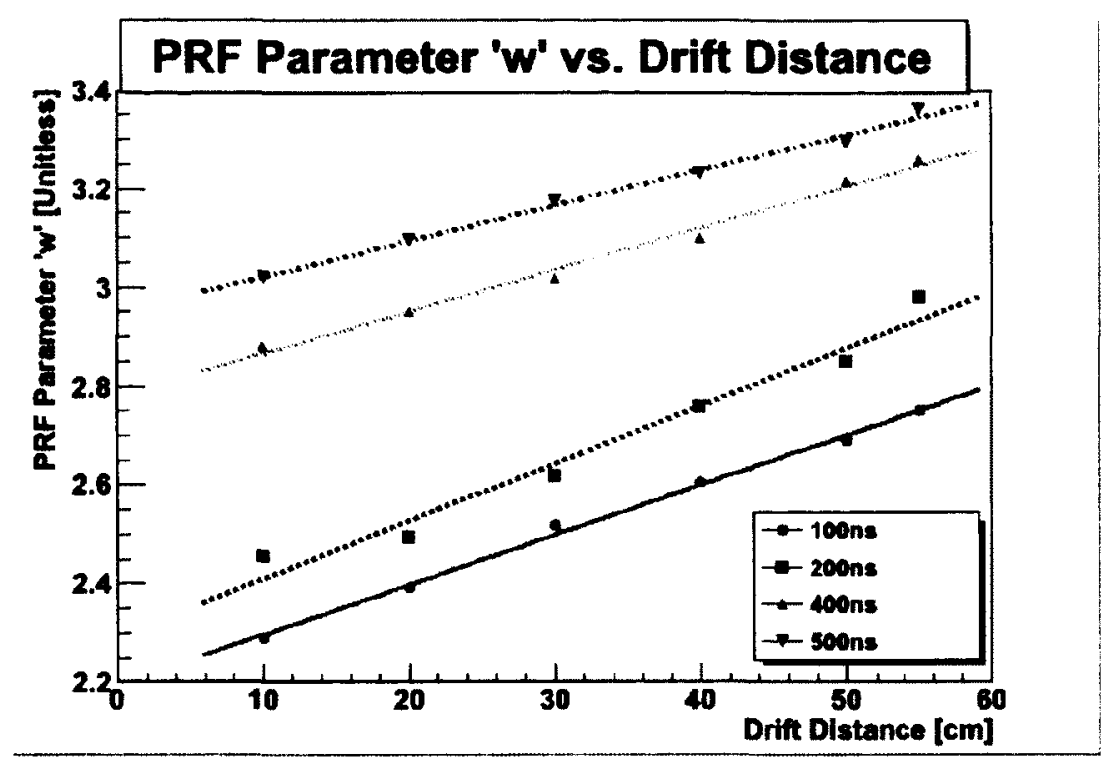

Figure 5.3: Calibration results for scans 1 through 4 of the PRF width parameter 'w'. These scans were analyzed using the QFM method. The width increases as a function of drift distance as can be expected due to diffusion. Note errors (statistical component only) on PRF parameters to small to be seen on graph.

Figures 5.3 and 5.5 show an increase in width parameter, w, with increased drift distance for both analysis techniques. These trends are again consistent with predictions resulting from diffusion. Both techniques produce PRFs with width parameters displaying a linear dependence on drift distance. However, the width parameter of the Reintegration Method PRF is significantly wider then the QFM PRF at all drift distances. The rate of increase in w is comparable between techniques, and across all shaping times.

Figures 5.6 and 5.7 show the PRFs determined for both analysis techniques and all shaping times. Figure 5.6 compares the PRFs at $10 \mathrm{~cm}$ of drift (shortest measurement). Figure 5.7 shows the same comparison at a drift distance of $55 \mathrm{~cm}$ (longest measurement). As can be seen in these figures the Reintegration Method produces a more dynamic PRF than the QFM. The vertical dashed lines represent the location and width of the average pad in the readout plane. The pad amplitude predicted by the PRF is the value of the PRF at the pad's midpoint. In figures 5.6 and 5.7 the 


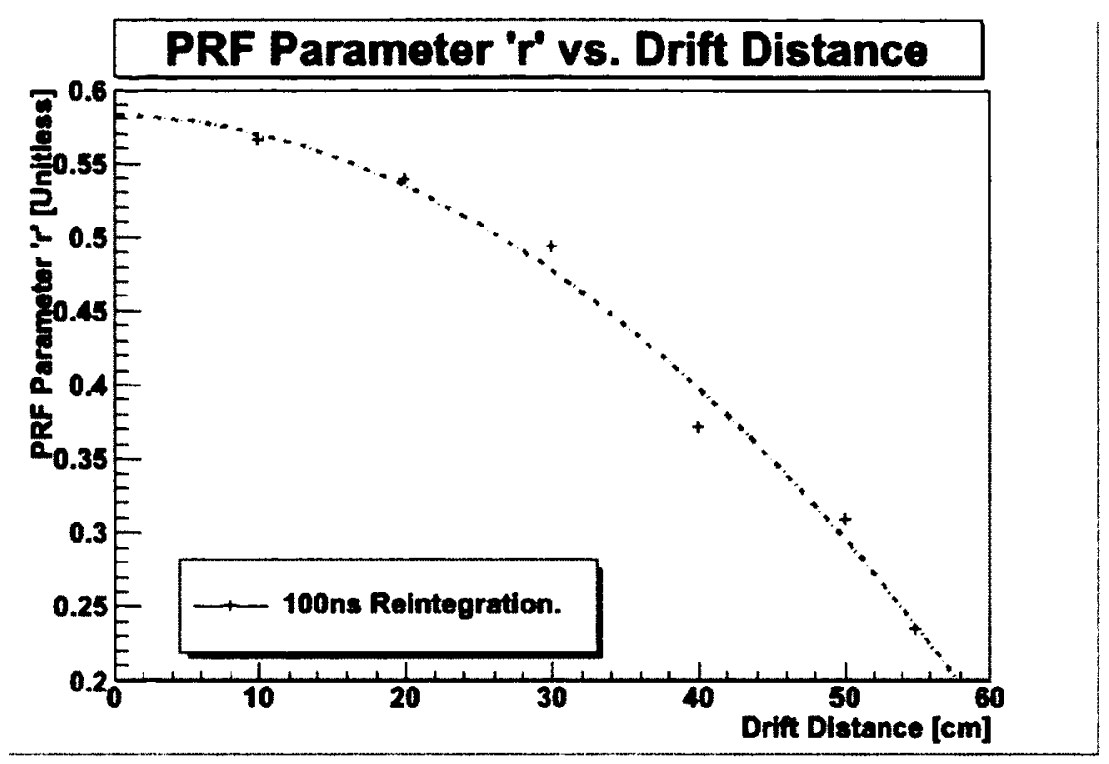

Figure 5.4: PRF parameter ' $r$ ' as a function of drift distance for scan 5 analyzed using the Reintegration Method. An ' $r$ ' value of 1 produces a fully Lorentzian function and 0 a fully Gaussian. The trend shows the PRF becoming more Gaussian like as drift distance increases. Note errors (statistical component only) on PRF parameters to small to be seen on graph.

neighbouring pad midpoints are at $\pm 3 \mathrm{~mm}$.

At short drift distances the RM PRF predicts an amplitude on the neighbouring pads of $\sim 12 \%$ that of the main pad. This value is in between the short shaping time (100 and $200 \mathrm{~ns}$ ) and long shaping time (400 and $500 \mathrm{~ns}$ ) QFM PRF predictions. At long drift distances the RM PRF matches the 500 ns QFM PRF with a neighbouring pad amplitude of $\sim 20 \%$ that of the main pad amplitude. At all drift distances the Reintegration Method PRF decreases to zero within $\sim 5 \mathrm{~mm}$. This is similar behaviour to the $100 \mathrm{~ns}$ and $200 \mathrm{~ns}$ QFM PRFs. This adaptability of the RM PRF may contribute to the Reintegration Method's improved transverse resolution measurements.

The PRF parameters determined in this section were used to complete the rest of the steps in the analysis pipeline. The parameters will also be useful to analyze data taken in the 7 modules test conducted in July of 2012 . 


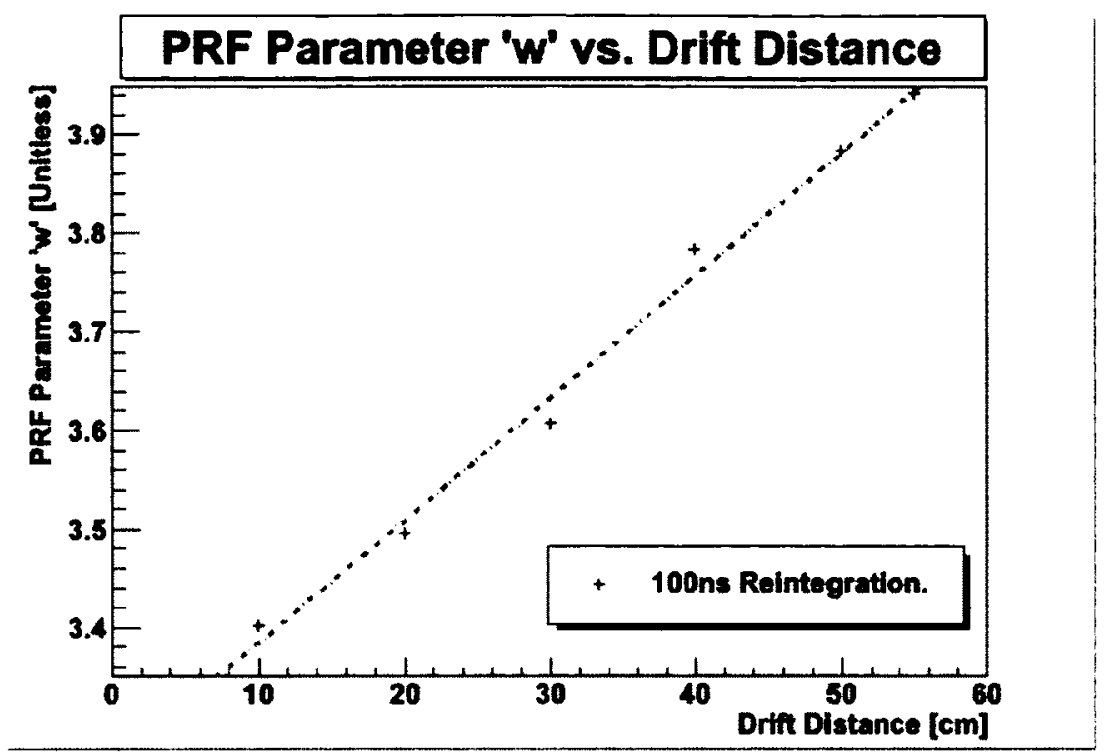

Figure 5.5: PRF parameter 'w' (width) as a function of drift distance for scan 5 analyzed using the Reintegration Method. The width increases as a function of drift distance as can be expected due to diffusion. Note errors (statistical component only) on PRF parameters to small to be seen on graph.

\subsection{Relative Tracking Efficiency}

The relative tracking efficiency (RTE) of a run is defined as the ratio of events which pass the event selection cuts (details of cuts given in appendix A) to the total number of events taken in that run. Table 5.1 and table 5.2 summarize the RTE for each run after the application of event cuts for the QFM and Reintegration Methods, respectively. An average RTE of $\sim 62 \%$ was observed for the Reintegration Method. The RTE for the QFM method varied depending on shaping time. The average RTE for the QFM was $\sim 58 \%$.

The measured resolution of a run can be artificially improved by decreasing the number of events which pass the event selection cuts. By allowing only those events which have a high signal to noise ratio to be analyzed the measured resolution would not be representative of the detectors abilities to measure the average track. Since the RTE for the Reintegration Method was similar to that of the QFM RTE, we can 


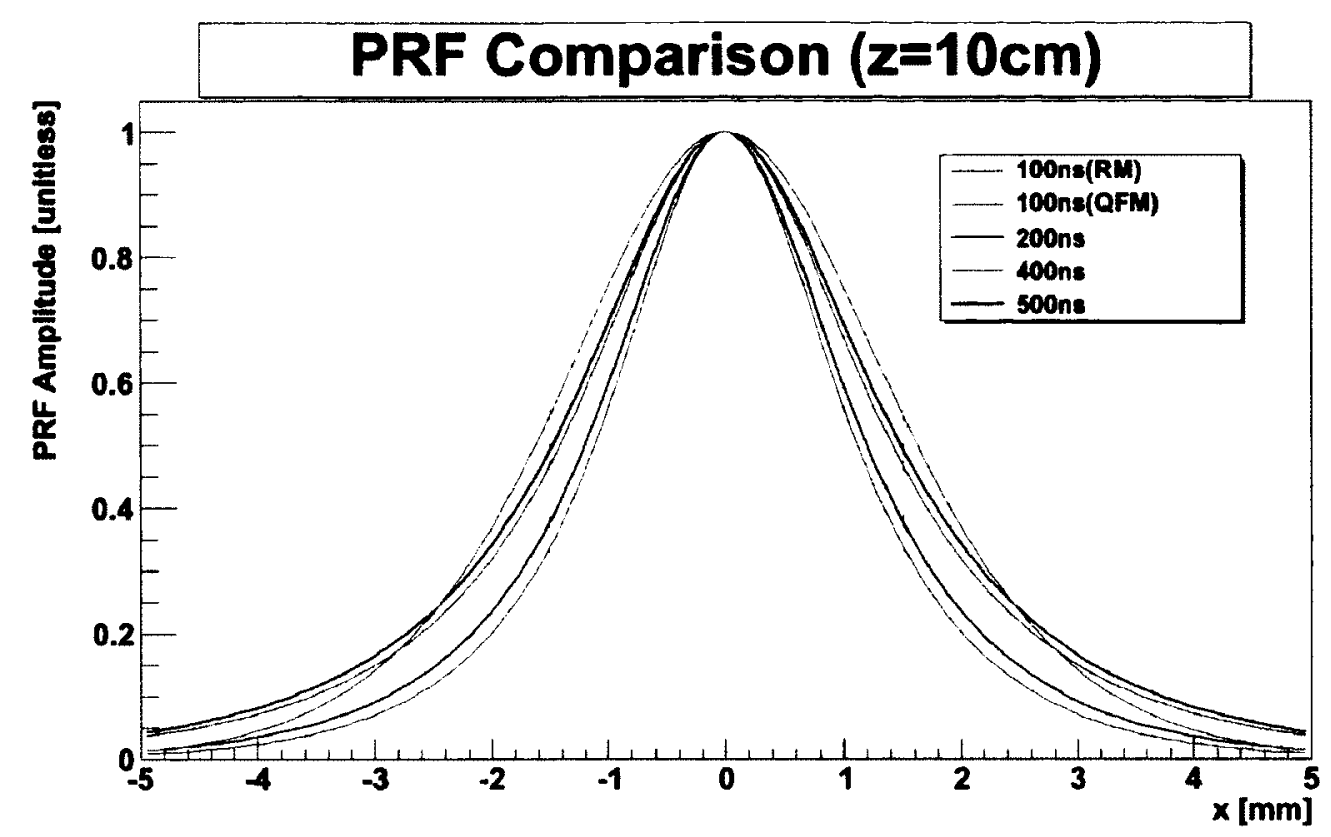

Figure 5.6: Comparison of the PRFs, at short drift distance, determined for both analysis techniques and all shaping times. The vertical dashed lines represent the average pad width.

be confident that the resolutions measured using the Reintegration Method were not artificially improved through too restrictive event selection.

\subsection{Bias Correction}

The bias corrections were calculated as described in section 4.3.3. The second and third consistency checks (also described in section 4.3.3) of the data were performed after applying the corrections to the track fitting procedure. In order to quantitatively express whether the bias correction had indeed decreased the position dependent biases in track position determination, the weighted average of the absolute value of row residuals and weighted standard deviation of the row residuals were calculated from the separate row residual scatter plots.

The weighted means and standard deviations (see figure5.8), for the row residuals both before and after application of the bias correction, were collected and displayed 


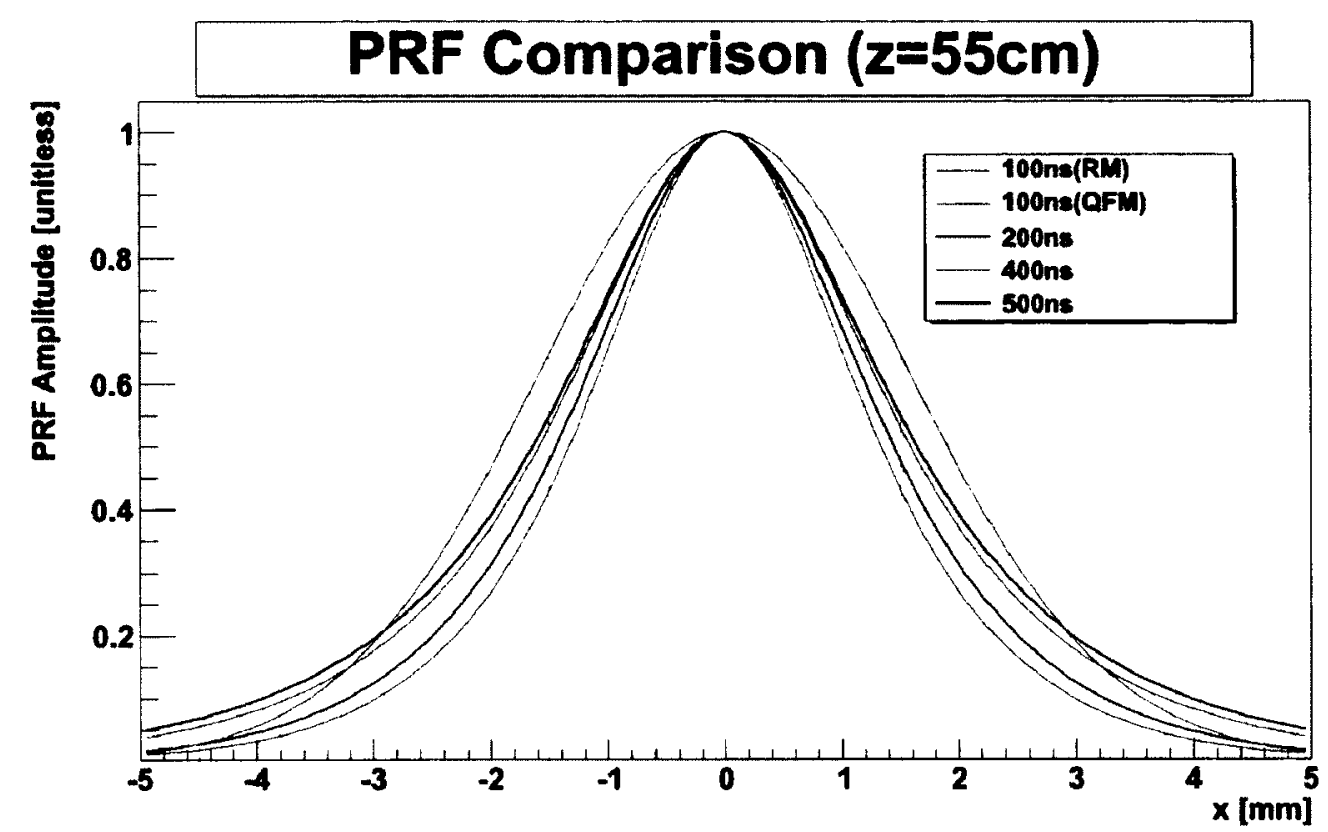

Figure 5.7: Comparison of the PRFs, at long drift distance, determined for both analysis techniques and all shaping times. The vertical dashed lines represent the average pad width.

in the bar graphs of weighted mean (top) and standard deviation (bottom) versus row number. As the graphs show, there was a significant reduction in position dependent biases in all rows.

\subsection{Resolution Measurements}

After the calculation and application of the bias corrections, the resolution for each run was measured. The method for determining the resolution is described in section 4.4. The measured resolution values with uncertainty are summarized in table 5.3. The error values included in the table were taken from the uncertainty on the fit to the global residual distribution for that run as described in section 4.4 .

Each scan was used to plot a graph of measured resolution versus drift distance, and equation 4.14 fitted to the measurements. These graphs were collected and plotted together in figure 5.9. Among the measurements of $\mathrm{r} \phi$ resolution obtained 


\begin{tabular}{|c|c|c|c||c|c|c|c|}
\hline Scan & Run \# & RTE & Percentage & Scan & Run \# & RTE & Percentage \\
\hline $1(100 \mathrm{~ns})$ & $1215(10 \mathrm{~cm})$ & 2851 & 57.0 & $3(400 \mathrm{~ns})$ & $1217(10 \mathrm{~cm})$ & 2731 & 54.6 \\
\hline & $1222(20 \mathrm{~cm})$ & 2867 & 57.3 & & $1224(20 \mathrm{~cm})$ & 2882 & 57.6 \\
\hline & $1227(30 \mathrm{~cm})$ & 2878 & 57.6 & & $1229(30 \mathrm{~cm})$ & 2862 & 57.2 \\
\hline & $1232(40 \mathrm{~cm})$ & 2932 & 58.6 & & $1234(40 \mathrm{~cm})$ & 2910 & 58.2 \\
\hline & $1237(50 \mathrm{~cm})$ & 3076 & 61.5 & & $1239(50 \mathrm{~cm})$ & 2950 & 59.0 \\
\hline & $1242(55 \mathrm{~cm})$ & 2863 & 57.3 & & $1244(55 \mathrm{~cm})$ & 2494 & 59.0 \\
\hline & Average & $\mathbf{2 9 1 1}$ & $\mathbf{5 8 . 2}$ & & Average & $\mathbf{2 8 8 0}$ & $\mathbf{5 7 . 6}$ \\
\hline $2(200 \mathrm{~ns})$ & $1216(10 \mathrm{~cm})$ & 2720 & 54.4 & $4(500 \mathrm{~ns})$ & $1218(10 \mathrm{~cm})$ & 2871 & 57.4 \\
\hline & $1223(20 \mathrm{~cm})$ & 2797 & 55.9 & & $1225(20 \mathrm{~cm})$ & 2891 & 57.8 \\
\hline & $1228(30 \mathrm{~cm})$ & 2711 & 54.2 & & $1230(30 \mathrm{~cm})$ & 2878 & 57.6 \\
\hline & $1233(40 \mathrm{~cm})$ & 2862 & 57.2 & & $1235(40 \mathrm{~cm})$ & 2948 & 59.0 \\
\hline & $1238(50 \mathrm{~cm})$ & 2970 & 59.4 & & $1240(50 \mathrm{~cm})$ & 2905 & 58.1 \\
\hline & $1243(55 \mathrm{~cm})$ & 2850 & 57.0 & & $1245(55 \mathrm{~cm})$ & 2843 & 56.9 \\
\hline & Average & $\mathbf{2 8 1 8}$ & $\mathbf{5 6 . 4}$ & & Average & $\mathbf{2 8 8 9}$ & $\mathbf{5 7 . 8}$ \\
\hline
\end{tabular}

Table 5.1: The relative tracking efficiency (RTE) of events per run using the QFM analysis technique. Each run was made up of 5000 events.

using the QFM the optimal shaping time depends on the drift distance. The 400 and 500 ns shaping time measurements provided the best final resolution measurement. This observation was in agreement with previous beam test results [6]. The $100 \mathrm{~ns}$ Reintegration Measurements improved the $\mathrm{r} \phi$ resolution at all drift distances when compared to the QFM measurements. 


\begin{tabular}{|c|c|c|c|}
\hline Run \# & Drift $(\mathrm{cm})$ & Accepted & Percentage \\
\hline 1214 & 10 & 3003 & 62.3 \\
\hline 1221 & 20 & 3065 & 61.7 \\
\hline 1226 & 30 & 3042 & 62.2 \\
\hline 1298 & 40 & 2969 & 62.1 \\
\hline 1299 & 50 & 2933 & 61.7 \\
\hline 1300 & 55 & 2990 & 61.3 \\
\hline Average & & $\mathbf{3 0 9 4}$ & $\mathbf{6 1 . 9}$ \\
\hline
\end{tabular}

Table 5.2: The relative tracking efficiency (RTE) per run using the RM analysis technique. Each run was made up of 5000 events. The average RTE was $\sim 62 \%$.

\subsection{Extrapolation of Resolution Results to ILC Conditions}

The ILD-TPC design places strict requirements on the TPCs single hit $r \phi$ resolution (see table 1.1). The single hit $\mathrm{r} \phi$ resolution is required to be $<100 \mu \mathrm{m}$ after 2 $m$ of drift in order to achieve the order of magnitude improvement in transverse momentum resolution. From figure 5.9 it would appear these requirements have not been met since the measured resolution is greater then $100 \mu \mathrm{m}$ at less then $50 \mathrm{~cm}$ of drift. However, these measurements were made using only a $1 \mathrm{~T}$ magnetic field which results in a transverse diffusion coefficient of $94.179 \mu \mathrm{m} / \sqrt{\mathrm{cm}}$. The ILC will have a $3.5 \mathrm{~T}$ magnetic field which will reduce the transverse diffusion coefficient to $30.68 \mu \mathrm{m} / \sqrt{\mathrm{cm}}$. Using this value of diffusion coefficient and the values of $N_{\text {eff }}$ and $\sigma_{0}$ extracted from the fit an extrapolation of the Reintegration Methods resolution to $2 \mathrm{~m}$ drift distance can be made. This extrapolation is shown in figure 5.10 . The shaded area is one standard deviation.

Figure 5.10 shows that the single hit $r \phi$ resolution, at a drift distance of $2 \mathrm{~m}$, 
achieved using the Reintegration method is $89.8 \pm 4 \mu \mathrm{m}$. This demonstrates the Reintegration Method's ability to meet the ILD-TPC single hit $r \phi$ resolution requirements. 


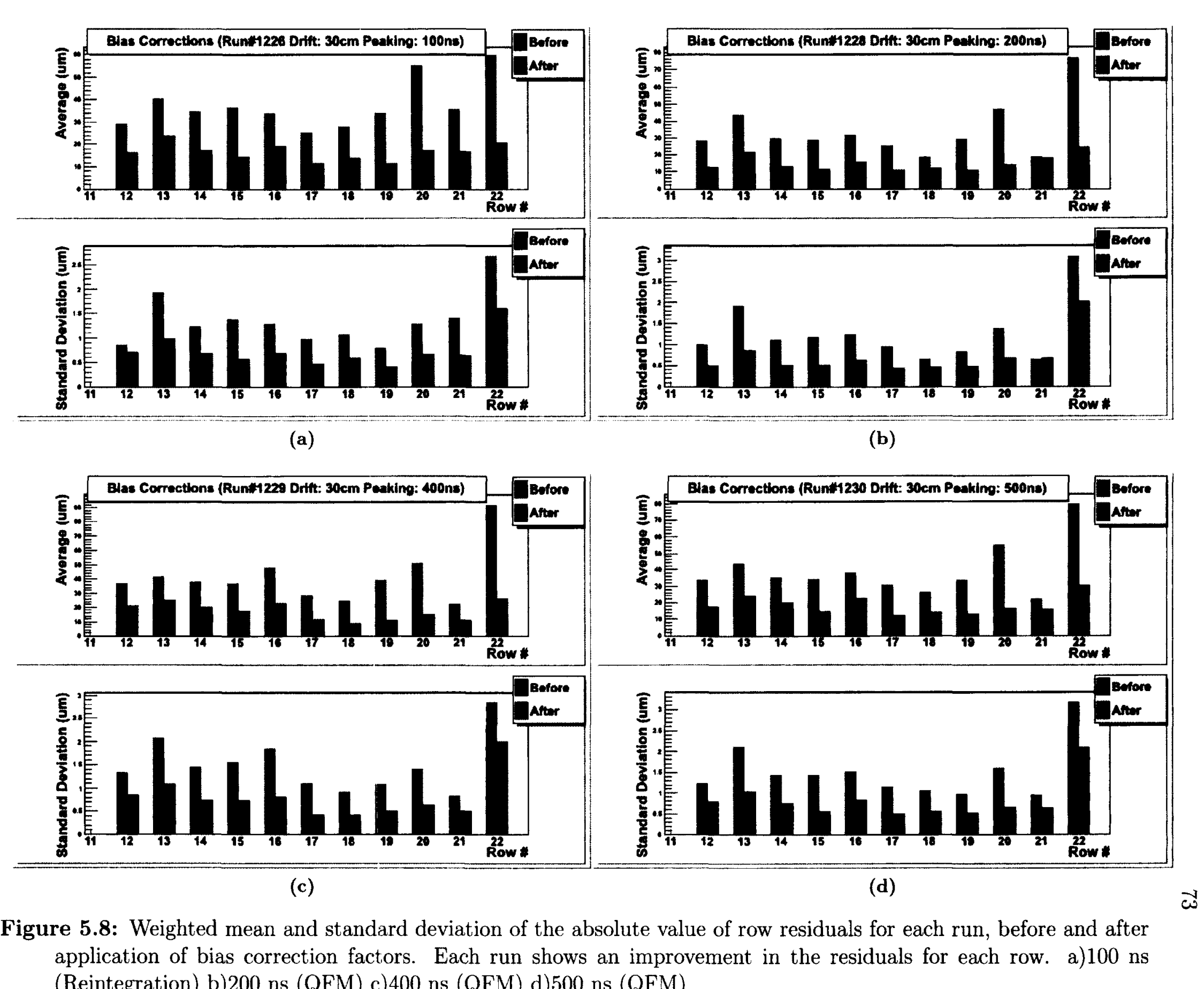




\begin{tabular}{|c|c|c|c|c|c|c|c|}
\hline Scan\# & Run\# & $\operatorname{Drift}[\mathrm{cm}]$ & $\sigma_{t r}[\mu \mathrm{m}]$ & Scan\# & Run\# & Drift $[\mathrm{cm}]$ & $\sigma_{t r}[\mu \mathrm{m}]$ \\
\hline \multirow[t]{6}{*}{1 (QFM) } & 1218 & 10 & $85.5 \pm 0.5$ & $3(\mathrm{QFM})$ & 1216 & 10 & $78 \pm 0.5$ \\
\hline & 1225 & 20 & $97.2 \pm 0.6$ & & 1223 & 20 & $95 \pm 0.5$ \\
\hline & 1230 & 30 & $112.7 \pm 0.7$ & & 1228 & 30 & $112.1 \pm 0.7$ \\
\hline & 1235 & 40 & $122.5 \pm 0.7$ & & 1233 & 40 & $123.5 \pm 0.7$ \\
\hline & 1240 & 50 & $135.4 \pm 0.8$ & & 1238 & 50 & $138.4 \pm 0.8$ \\
\hline & 1245 & 55 & $139.9 \pm 0.8$ & & 1243 & 55 & $145.6 \pm 0.8$ \\
\hline \multirow[t]{6}{*}{$2(\mathrm{QFM})$} & 1217 & 10 & $85.1 \pm 0.5$ & $4(\mathrm{QFM})$ & 1215 & 10 & $79.2 \pm 0.5$ \\
\hline & 1224 & 20 & $98.1 \pm 0.6$ & & 1222 & 20 & $94.7 \pm 0.6$ \\
\hline & 1229 & 30 & $111.8 \pm 0.7$ & & 1227 & 30 & $114.3 \pm 0.7$ \\
\hline & 1234 & 40 & $122.9 \pm 0.7$ & & 1232 & 40 & $126.3 \pm 0.7$ \\
\hline & 1239 & 50 & $136.6 \pm 0.7$ & & 1237 & 50 & $141.1 \pm 0.8$ \\
\hline & 1244 & 55 & $139.8 \pm 0.8$ & & 1242 & 55 & $149.5 \pm 0.9$ \\
\hline \multirow[t]{6}{*}{$5(\mathrm{RM})$} & 1214 & 10 & $73.7 \pm 0.4$ & & & & \\
\hline & 1221 & 20 & $87.8 \pm 0.5$ & & & & \\
\hline & 1226 & 30 & $105.3 \pm 0.6$ & & & & \\
\hline & 1298 & 40 & $111.6 \pm 0.6$ & & & & \\
\hline & 1299 & 50 & $118.0 \pm 0.7$ & & & & \\
\hline & 1300 & 55 & $123.8 \pm 0.7$ & & & & \\
\hline
\end{tabular}

Table 5.3: Summary of measured resolution as a function of drift distance for scans 1 through 4(above) and scan 5(below). 


\section{Resolution v. Drift Distance (All Scans)}

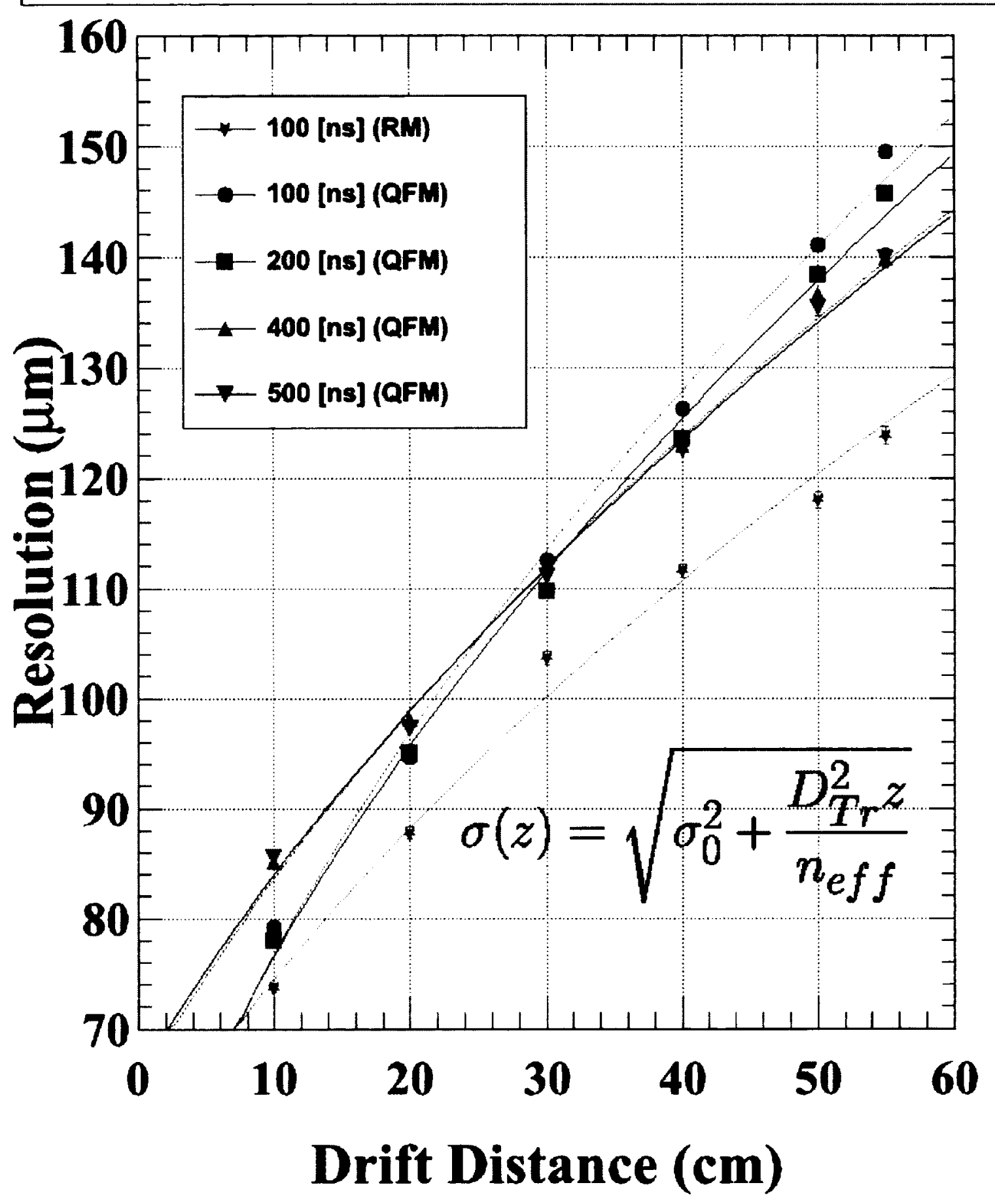

Figure 5.9: Measured resolution for scans 1 through 4 analyzed using the QFM method and scan 5 analyzed using the Reintegration Method. The resolution dependence on drift distance is consistent with diffusion. 


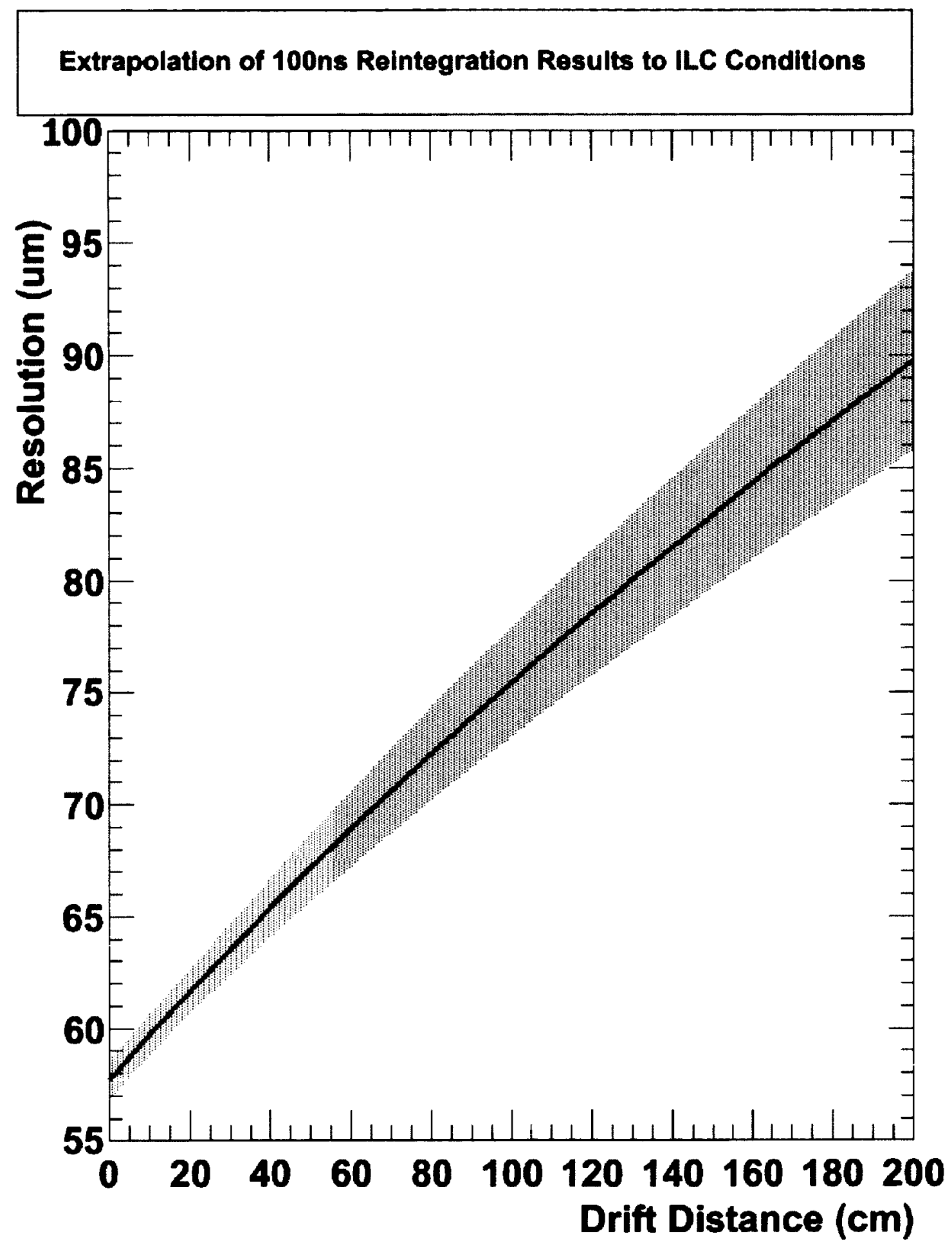

Figure 5.10: Extrapolation of resolution measurements of scan 5 (100 ns data, analyzed using RM technique) analyzed using the Reintegration Method to ILC conditions. The shaded region shows the one sigma error bound. 


\section{Chapter 6}

\section{Conclusion}

For this thesis I worked on the development of a new pulse analysis technique, the Reintegration Method, which is capable of achieving the ILD-TPC single hit $\mathrm{r} \phi$ resolution requirements while employing relatively short 100 ns shaping times. Previously, a relatively long main amplifier shaping time of $500 \mathrm{~ns}$ was needed to integrate all electrons contributing to the pad preamplifier pulse. However, by integrating a 100 ns shaping time FADC pulse over a fixed window the Reintegration Method is able to emulate the long shaping time pad amplitudes.

The relatively long shaping times previously required would be detrimental to longitudinal resolution, and two hit resolving power. The Reintegration Method, however, is capable of achieving the single hit $\mathrm{r} \phi$ resolution requirements without compromising longitudinal resolution and two hit resolving power. The measurements of the single hit $\mathrm{r} \phi$ resolution are summarized in table 5.3. As the table shows, the Reintegration Method provides significant improvements at all drift distances between $10 \mathrm{~cm}$ and $55 \mathrm{~cm}$ when compared with existing pulse height techniques.

Fitting equation 4.14 to the measured resolutions obtained using both pulse analysis methods shows the dependence of resolution on drift distance is consistent with being only limited by diffusion and electron statics. Fitting equation 4.14 to the Reintegration Method resolution measurements provides the fit parameters $\sigma_{0}=57.7 \pm 0.9$ 
and $n_{\text {eff }}=39.8 \pm 0.6$. These parameters were used to extrapolate the $\mathrm{r} \phi$ resolution to $2 \mathrm{~m}$ (see figure 5.10$)$ of drift under the ILC conditions $\left(\mathrm{B}=3.5 \mathrm{~T}, \mathrm{D}_{\operatorname{Tr}}=24.9\right.$ $\mu \mathrm{m} / \sqrt{\mathrm{cm}}$ ). The extrapolated resolution at $2 \mathrm{~m}$ drift is $89.8 \pm 4 \mu \mathrm{m}$ which more than fulfils the ILD-TPC transverse resolution requirement of $<100 \mu \mathrm{m}$. This demonstrates that the Reintegration method is capable of achieving the ILD-TPC single hit $\mathrm{r} \phi$ resolution requirements.

The validity of the results obtained has been confirmed through three validation tests of the data and analysis techniques. The first check focused on the dependence of the PRF parameters on drift distance. The second and third tests examined the distribution of row residuals before and after the application of bias corrections. The offset from zero, and the width of the distribution were both considered.

The PRF parameters were shown to vary smoothly (figures 5.2, 5.3, 5.4, and 5.5) with drift distance. The mixing parameter $\mathrm{r}$ decreased and the width parameter $\mathrm{w}$ increased with increasing drift distance. These behaviours were consistent with what was to be expected due to the increase in diffusion resulting from increased drift distances.

The results of the second and third consistency checks also confirmed the validity of the analysis results. There was a decrease in residual distribution offset for all 11 pad rows used in the resolution measurement studies. There was also a decrease in the widths of the distributions for all row residual distributions.

Tables 5.1 and 5.2 further confirm the validity of the $\mathrm{r} \phi$ resolution measurements obtained using the Reintegration Method. The relative tracking efficiency (RTE) shown in these tables demonstrate that the Reintegration Method improved the RTE of short shaping time data. The RTE were comparable between the Reintegration Method and QFM which means the improved resolutions of the Reintegration Method were not necessarily the result of overly restrictive event selection cuts. 
Although a method has been developed which is capable of achieving the ILDTPC transverse resolution goals using short shaping times there is plenty of work left to be done. The Reintegration Method does not provide a time measurement of the pulse. A suitable method must be developed which can use short 100 ns shaping time pulses to achieve the ILD-TPC rz resolution goals (table 1.1). The Reintegration Method could be further improved by optimizing the integration width for longer drift distances instead of for $30 \mathrm{~cm}$ of drift as was presently done. 


\section{References}

[1] Technical Report No. ATLAS-CONF-2012-093, CERN, Geneva (unpublished).

[2] T. Abe et al., The International Large Detector: Letter of Intent, http://ilcild. org/documents/ild-letter-of-intent/LOI\%20Feb2010.pdf/view.

[3] LCTPC Collaboration, Report to the Desy RPC 2010, http://ilcdoc. linearcollider.org/record/37960?ln=en, 2012.

[4] F. Sauli and A. Sharma, MICROPATTERN GASEOUS DETECTORS, Annual Review of Nuclear and Particle Science 49, 341 (1999).

[5] M. Dixit, Development of high resolution Micro-Pattern Gas Detectors with wide readout pads,Journal of Instrumentation, (http://stacks.iop.org/17480221/5/i=03/a=P03008), 5, P03008 (2010).

[6] P. Colas, First test results from a Micromegas large TPC prototype, Nuclear Instruments and Methods in Physics Research Section A: Accelerators, Spectrometers, Detectors and Associated Equipment 623, 100 (2010), International Conference on Technology and Instrumentation in Particle Physics.

[7] R. Fernow, Introduction to experimental particle physics, (Cambridge University Press, Cambridge, England, 1986).

[8] W. Blum et al., Particle Detection with Drift Chambers, (Springer-Verlag Berlin Heidelberg, Berlin, Germany, 2008).

[9] J. Beringer et al. (Particle Data Group), Phys. Rev. D86, 010001 (2012), .

[10] S. Biagi, Monte Carlo simulation of electron drift and diffusion in counting gases under the influence of electric and magnetic fields, Nuclear Instruments and Methods in Physics Research Section A: Accelerators, Spectrometers, Detectors and Associated Equipment 421, 234 (1999). 
[11] N. M. Isabell Melzer-Pellmann, Test Beams at DESY, http://adweb.desy.de/ -testbeam/, 2012.

[12] S. Amendolia et al., ExB and angular effects in the avalanche localization along the wire with cathode pad read-out, Nuclear Instruments and Methods in Physics Research 217, 317 (1983).

[13] F. Sauli, GEM: A new concept for electron amplification in gas detectors, Nucl.Instrum.Meth. A386, 531 (1997).

[14] Y. Giomataris, P. Rebourgeard, J. Robert, and G. Charpak, MICROMEGAS: A High granularity position sensitive gaseous detector for high particle flux environments, Nucl.Instrum.Meth. A376, 29 (1996).

[15] D. Karlen, P. Poffenberger, and G. Rosenbaum, TPC performance in magnetic fields with GEM and pad readout, Nuclear Instruments and Methods in Physics Research Section A: Accelerators, Spectrometers, Detectors and Associated Equipment 555, 80 (2005).

[16] M. Dixit, J. Dubeau, J. Martin, and K. Sachs, Position sensing from charge dispersion in micropattern gas detectors with a resistive anode, Nucl.Instrum.Meth. A518, 721 (2004).

[17] M. Dixit and A. Rankin, Simulating the charge dispersion phenomena in micro pattern gas detectors with a resistive anode, Nucl.Instrum.Meth. A566, 281 (2006).

[18] J. Alozy et al., First Version of the PCMAG Field Map, www.eudet.org/e26/ e28/e182/e599/eudet-memo-2007-51.pdf, 2007.

[19] Madhu S. Dixit, Carleton University(Ottawa), personal communication, 2012.

[20] K. Boudjemline, M. Dixit, J.-P. Martin, and K. Sachs, Spatial resolution of a GEM readout TPC using the charge dispersion signal, Nucl.Instrum.Meth. A574, 22 (2007).

[21] A. Bellerive et al., Spatial resolution of a micromegas-TPC using the charge dispersion signal, eConf C050318, 0829 (2005).

[22] N. Abgrall et al., Time Projection Chambers for the T2K Near Detectors, Nucl.Instrum.Meth. A637, 25 (2011).

[23] Eric Delagnes, CEA(Saclay), personal communication, 2012. 
[24] R. Carnegie et al., Resolution studies of cosmic ray tracks in a TPC with GEM readout, Nucl.Instrum. Meth. A538, 372 (2005).

[25] D. Attie, Beam tests of Micromegas LC-TPC large prototype, JINST 6, C01007 (2011).

[26] R. Gluckstern, Uncertainties in track momentum and direction, due to multiple scattering and measurement errors, Nuclear Instruments and Methods 24, 381 (1963). 


\section{Appendix A}

\section{Event Selection}

There are six event selection cuts performed before an event is used in the analysis. These event cuts are applied before the PRF calibration, bias correction calculation, and resolution calculation steps of the analysis pipeline. This appendix will describe each event cut in order of its application.

\section{A.1 Central Columns Check}

As described in section 3.1 the readout module is positioned so the electron beam illuminates only the central columns. Any particle detected outside of the central columns is either not a beam particle or a scattered beam particle. Since we are interested in analyzing only well defined ( $5 \mathrm{GeV}$ electrons) beam particles, any events with particles outside the beam should be cut. This event selection cut only allows through events which contain a track which is fully contained within the central ten columns. To ensure the track is fully contained in the central region of the readout an event is discarded if signal is present in columns 31 and 42 . 


\section{A.2 Single Track Check}

The central columns check only ensures the signals in the central region are fully contained, it does not check the number of tracks contained. As the name implies the single track check ensures only a single track is contained in the central region. This is done by checking the number of pads in each row which contain signal. The charge from a single track rarely reach more then 5 pads in a single row. If there are more than 3 pad rows in a single event with more than 5 pads with signal then the event is discarded as a multitrack event.

\section{A.3 Event Veto}

The event veto check makes sure enough rows containing adequate signal to complete the track fit. For a row to have adequate signal a minimum number of pads must have an amplitude greater than a certain threshold. The amplitude threshold is set as a percentage of the primary pad amplitude. This percentage is stored in the code as the variable "kPercentNoise" and is set to a value of 0.05 . For a row to be considered as having adequate signal a minimum of two pads must have amplitudes above the threshold. For the event to pass this selection cut at least "minHitTrack" number of rows must contain enough signal. The variable "minHitTrack" is currently set to 6 .

\section{A.4 Amplitude Overflow}

The signals recorded by each pad are digitized using a 12-bit ADC. This gives the digitized pad pulses a range from 0 to 4096. To account for undershoots in the pulses the zero of the digitized pulse is set to 256 . This results a maximum pulse height of 3840. This value is stored in the code as "kMaxSignal" and any pulse greater than this value will be truncated. This will result in an artificially lowered calculated 
pad amplitude. A maximum pad amplitude, "kMaxAmplitude", is set to ensure these truncated pulses are not used. When using the pulse height analysis techniques (Either fit point maximum or quadratic fit maximum) "kMaxAmplitude" amplitude is set to 3840 . When using the pulse shape analysis technique (the Reintegration method) this value is set to 13000 . When an amplitude is being calculated using the Reintegration Method if any time bin in the ADC pulse has a value greater than "kMaxSignal" (meaning it has been artificially truncated), the amplitude is set to 99999. This will ensure the overflow event selection detects the event and discards it.

\section{A.5 Track Fit Failure}

A track is fit to the data using a $\chi^{2}$ minimization as described in section 4.2 . The $\chi^{2}$ is minimized using the Minuit minimization package. If a minimum is not found by Minuit then the track fit has failed for some reason. If this happens the event is discarded.

\section{A.6 Track Angle Cut}

The final event selection cut is applied after the track has been fit to the event data. If the fitted track has a $\phi$ angle, the angle the track makes with respect to the $y$ axis (see section 4.2), which is greater then 5 degrees the event is discarded. This track angle cut is applied to remove events suffering from the track angle effect which adversely affects the calculated detector resolution. 


\section{Appendix B}

\section{Scan and Run Parameters}

This appendix contains the parameters for each scan and its associated runs. Each scan contains six runs which are collections of 5000 events at fixed drift distances. The parameters changed between scans are,

1. Shaping Time $(100,200,400,500[\mathrm{~ns}])$

2. Zero Suppression (TRUE, FALSE)

The tables are divided into two groups. The first set of data was analyzed using the Quadratic Fit Method of amplitude calculation, and the second set was analyzed using the Reintegration Method. 


\begin{tabular}{|c|c|c|c|c|c|}
\hline \multicolumn{4}{|c|}{ Scan Parameters } & \multicolumn{2}{|c|}{ Run Parameters } \\
\hline Scan \# & Shaping Time[ns] & Zero Sup. & Sampling $[\mathrm{MHz}]$ & Run \# & $\mathrm{z}_{\text {drift }}[\mathrm{cm}]$ \\
\hline \multirow{6}{*}{1} & \multirow{6}{*}{500} & \multirow{6}{*}{ TRUE } & \multirow{6}{*}{25} & 1218 & 10 \\
\hline & & & & 1225 & 20 \\
\hline & & & & 1230 & 30 \\
\hline & & & & 1235 & 40 \\
\hline & & & & 1240 & 50 \\
\hline & & & & 1245 & 55 \\
\hline \multirow{6}{*}{2} & \multirow{6}{*}{400} & \multirow{6}{*}{ TRUE } & \multirow{6}{*}{25} & 1217 & 10 \\
\hline & & & & 1224 & 20 \\
\hline & & & & 1229 & 30 \\
\hline & & & & 1234 & 40 \\
\hline & & & & 1239 & 50 \\
\hline & & & & 1244 & 55 \\
\hline \multirow{6}{*}{3} & \multirow{6}{*}{200} & \multirow{6}{*}{ TRUE } & \multirow{6}{*}{25} & 1216 & 10 \\
\hline & & & & 1223 & 20 \\
\hline & & & & 1228 & 30 \\
\hline & & & & 1233 & 40 \\
\hline & & & & 1238 & 50 \\
\hline & & & & 1243 & 55 \\
\hline \multirow{6}{*}{4} & \multirow{6}{*}{100} & \multirow{6}{*}{ TRUE } & \multirow{6}{*}{25} & 1215 & 10 \\
\hline & & & & 1222 & 20 \\
\hline & & & & 1227 & 30 \\
\hline & & & & 1232 & 40 \\
\hline & & & & 1237 & 50 \\
\hline & & & & 1242 & 55 \\
\hline \multirow{6}{*}{5} & \multirow{6}{*}{100} & \multirow{6}{*}{ FALSE } & \multirow{6}{*}{25} & 1214 & 10 \\
\hline & & & & 1221 & 20 \\
\hline & & & & 1226 & 30 \\
\hline & & & & 1298 & 40 \\
\hline & & & & 1299 & 50 \\
\hline & & & & 1300 & 55 \\
\hline
\end{tabular}




\section{Appendix C}

\section{Resolution Definitions}

There are five types of resolution mentioned in this thesis, four spatial and one momentum. The four spatial resolutions are the single hit $\mathrm{r} \phi$ and $\mathrm{rz}$, and the two track $\mathrm{r} \phi$ and $\mathrm{rz}$. It is the goal of this thesis to develop a pulse analysis technique which achieves a single hit $\mathrm{r} \phi$ resolution of less than $100 \mu \mathrm{m}$ at $2 \mathrm{~m}$ of drift, without compromising the other spatial resolutions. The single hit $\mathrm{r} \phi$ resolution requirement is needed to attain a transverse momentum resolution of $2 \times 10^{-5}(\mathrm{GeV} / \mathrm{c})^{-1}$. The meaning of the different spatial resolutions and the effect of single hit $\mathrm{r} \phi$ on the transverse momentum resolution will be discussed in this appendix.

The spatial resolutions are divided into two groups single hit and two track resolutions. The single hit resolution refers to the resolution associated with a single pad rows measurement of track position. The two track resolution is the degree to which the detector is capable of resolving two tracks in close proximity to each other.

Both the single hit and two track resolutions have two directions associated with them, $\mathrm{r} \phi$ and $\mathrm{rz}$. The ILD-TPC will be a cylindrical TPC so it is easy to describe positions or directions using a cylindrical coordinate system. The directions $\mathrm{r} \phi$ and rz refer to directions in this coordinate system. The $\mathrm{r} \phi$ resolution refers to resolution in the direction transverse to the axis of the TPC, that is, transverse to the readout plane. The $\mathrm{rz}$ resolution is in the direction parallel to the axis of the TPC. That is, 
the direction from the readout plane into the gas volume.

Putting the concepts together one arrives at the following definitions of the spatial resolutions:

- Single Hit $\mathrm{r} \phi$ : Resolution associated with determination of the $\mathrm{x}$ coordinate of the track position for a pad row.

- Single Hit rz: Resolution associated with determination of the $z$ coordinate of the track position for a pad row.

- Two Track $\mathrm{r} \phi$ : Ability of the detector to resolve two closely separated tracks in the transverse direction.

- Two Track rz: Ability of the detector to resolve two closely separated tracks in the longitudinal direction.

There is no resolution associated with the measurement of the $\mathrm{y}$ coordinate of the track position. This is because the y coordinate is measured from the position of the pad row.

How the single hit $\mathrm{r} \phi$ resolution impacts the transverse momentum resolution can be seen from a simple example. Assume a charged particle travels through the gas volume of the TPC in the transverse direction (ie. at constant $\mathrm{z}$ ). The particle track will be a segment of a circular arc. The $\mathrm{N}$ pads rows on the detector readout will sample this path at $\mathrm{N}$ points. Each of the $\mathrm{N}$ points will have an error associated to it equal to the single hit $\mathrm{r} \phi$ resolution. A circle can be fit to these $\mathrm{N}$ points and the radius of curvature of the circle related to the particle momentum through equation C.1, where $R$ is the radius of curvature, $p$ is the particles momentum, $q$ the charge of the particle, and $\mathrm{B}$ the strength of the magnetic field.

$$
R=\frac{p}{q B}
$$


The error on the $\mathrm{N}$ points will propagate through the circle fit to the radius of curvature and then to the determination of the particle momentum.

A more rigorous derivation of the errors in the track fit, which accounts for energy loss of the charge particle, multiple scattering in the gas, and a particle trajectory not confined to the transverse plane was carried out by Gluckstern [26]. The Gluckstern formula used to calculate the transverse momentum resolution given the single hit $\mathrm{r} \phi$ resolution, $\sigma_{r \phi}$, (and other detector parameters) is shown in equation C.2.

$$
\frac{\partial P_{T r}}{P_{T r}^{2}}=\frac{\sigma_{r \phi}}{L^{2}} \frac{1}{0.3 B} \sqrt{\frac{720}{N+4}}
$$

In the Gluckstern formula $\mathrm{B}$ is the strength of the magnetic field (3.5 $\mathrm{T}$ at the ILDTPC), $\mathrm{L}$ is the track length (about the radius of the ILD-TPC $2 \mathrm{~m}$ ), and $\mathrm{N}$ is the number of track points (on the order of 200 for the ILD-TPC). 